HYDROLOGIC DATA FOR THE BIG SPRING BASIN, CLAYTON COUNTY, IOWA, WATER YEAR 1988

By Stephen J. Kalkhoff

U.S. GEOLOGICAL SURVEY

Open-File Report 89-230

Prepared in cooperation with

IOWA DEPARTMENT OF NATURAL RESOURCES (GEOLOGICAL SURVEY BUREAU)

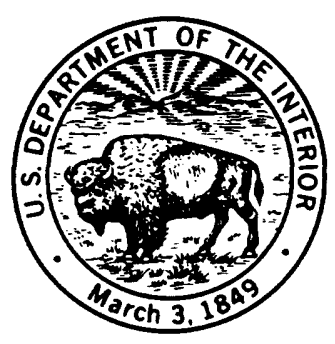

Iowa City, Iowa 


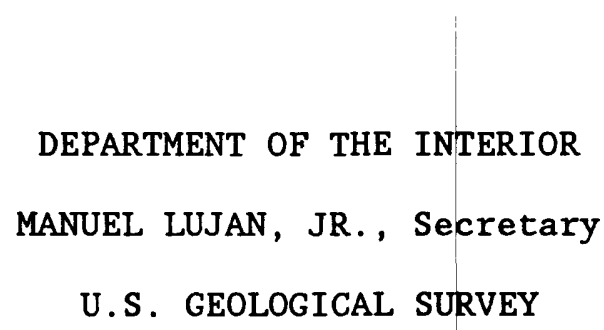

Dallas L. Peck, Director

For additional information write to:

U.S. Geological Survey Water Resources Division Rm. 269, Federal Building 400 South Clinton St. Iowa City, Iowa 52244
Copies of this report can be purchased from:

Books and Open-File Reports

U.S. Geological Survey

Federal Center

Box 25425

Denver, Colorado 80225 


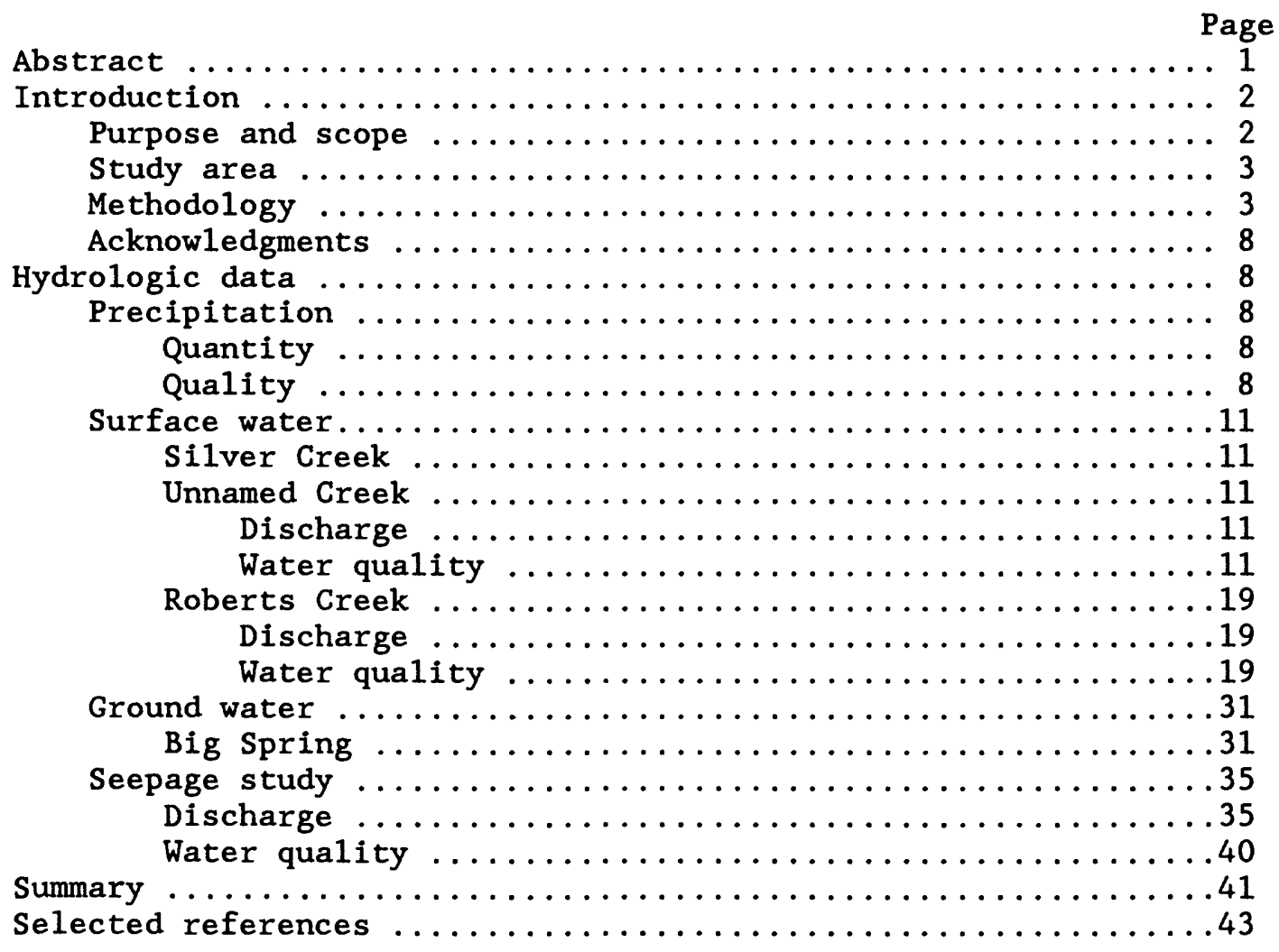

ILLUSTRATIONS

Figure 1. Map showing:

Page

1. Location of the study area and sampling sites...... 4

Figures 2.-8. Graphs showing:

2. Daily precipitation, daily mean specific conductance discharge, water temperature, and daily median $\mathrm{pH}$ at site BOOGD, Unnamed Creek near Luana, Iowa, March-September, $1988 \ldots \ldots \ldots \ldots \ldots \ldots \ldots \ldots$

3. Daily precipitation, daily mean specific conductance, discharge, water temperature, and daily median $\mathrm{pH}$ at site RC2, Roberts Creek above Saint 01af, Iowa, March-September, 1988........23 
4. Discharge and suspended sediment concentrations at sites BOOGD, Unnamed Creek near Luana, and RC2, Roberts Creek above Saint Olaf, Iowa,

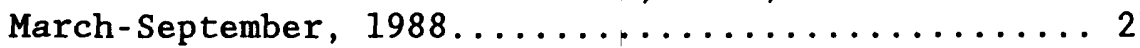

5. Diurnal variation of selected physical and chemical constituents at site RC2, Roberts Creek above Saint Olaf, Iowa, June 23-24, 1988............ 30

6. Weekly precipitation, daily mean specific conductance gage height, water temperature, and daily median $\mathrm{pH}$ at Big Spring, Clayton County, Iowa,

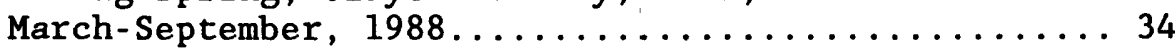

7. Discharge, total dissolved nitrogen concentration, and total dissolved nitrogen load in Roberts Creek,

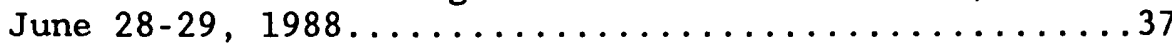

8. Discharge, total dissolved nitrogen concentration, and total dissolved nitrogen load in East Fork Silver Creek

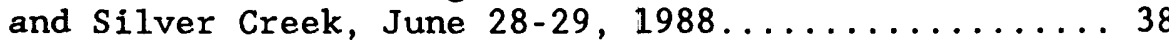

\section{TABLES}

Table 1. Location and drainage area of sampling sites ....... 5

2. Sample preparation and chemical analysis procedures..... 7

3. Statistical summary of precipitation quantity and quality at Big Spring, water year October 1987 to

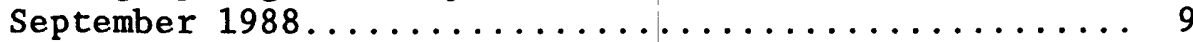

4. Accumulated daily rainfal1, water year October 1987

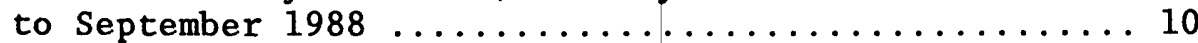

5. Daily mean discharge at site L23, Silver Creek near Luana, Iowa, water year October 1987 to September $1988 \ldots \ldots 12$

6. Daily mean discharge and specific conductance at site BOOGD, Unnamed Creek near Luana, Iowa water year October 1987 to September $1988 \ldots \ldots \ldots \ldots \ldots 13$

7. Daily mean water temperature and median $\mathrm{pH}$ at site BOOGD, Unnamed Creek near Luana, Iowa water year October 1987 to September $1988 \ldots \ldots \ldots \ldots 15$

8. Field measurements at monthly monitoring sites in the Big Spring Basin..................... 16

9. Concentrations of major ions at monthly monitoring sites in the Big Spring basin.......... 17

10. Selected nitrogen, phosphorus and carbon species at monthly monitoring sites in the Big Spring basin..... 18

11. Selected pesticides at monthly monitoring sites in the Big Spring basin.................... 20 
TABLES - - Continued

12. Instantaneous discharge and suspended sediment concentrations at site BOOGD, Unnamed Creek near Luana, Iowa...................... 21

13. Daily mean discharge and specific conductance at site RC2, Roberts Creek above Saint Olaf, Iowa water year October 1987 to September $1988 \ldots \ldots \ldots \ldots 22$

14. Daily mean water temperature and median $\mathrm{pH}$ at site RC2, Roberts Creek above Saint Olaf, Iowa water year October 1987 to September $1988 \ldots \ldots \ldots \ldots 24$

15. Instantaneous discharge and suspended sediment concentrations at site RC2, Roberts Creek above

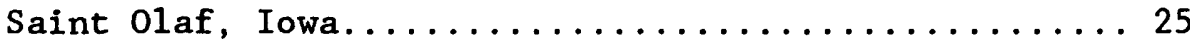

16. Diurnal variation of selected physical and chemical constituents at site RC2, Roberts Creek above Saint 0laf, Iowa........................ 29

17. Daily mean specific conductance and water temperature at Big Spring, water year October 1987 to

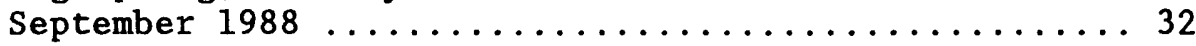

18. Daily median $\mathrm{pH}$ at Big Spring, water year October 1987 to

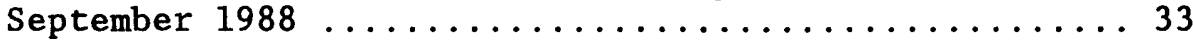

19. Field measurements and chemical analyses of samples from streams in the Big Spring Basin, during low-flow

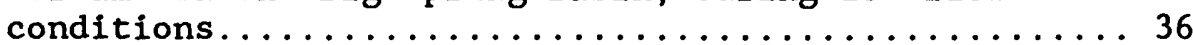

20. Seepage in a selected reach of Roberts Creek.......... 39 


\section{CONVERSION FACTORS}

For use of readers who prefer to use International System (SI) units, rather than the inch-pound terms used in this report, the following conversion factors may be used:

\section{Multiply inch-pound units}

foot ( $f t)$

cubic foot per second $(\mathrm{ft} 3 / \mathrm{s})$

million gallons per day (Mgal/d)

inch (in.)

mile (mi)

square mile (mi2)
By

\section{To obtain SI units}

0.3048

0.02832

0.0438

25.40

1.609

2.590 meter (m)

cubic meter per second $(\mathrm{m} 3 / \mathrm{s})$

cubic meter per second $(\mathrm{m} 3 / \mathrm{s})$

millimeter (mm)

kilometer $(\mathrm{km})$

square kilometer ( $\mathrm{km} 2)$

Temperature in degrees Fahrenheit $(F)$ as follows:

$F=(1.8 \times C)+32$ 


\title{
HYDROLOGIC DATA FOR THE BIG SPRING BASIN, CLAYTON COUNTY, IOWA, WATER YEAR 1988
}

\author{
By Stephen J. Kalkhoff
}

\section{ABSTRACT}

Hydrologic data was collected during the 1988 water year in cooperation with the Iowa Department of Natural Resources Geological Survey Bureau in the Big Spring ground-water basin located in Clayton County, Iowa. Information on precipitation, streams, and ground water was collected in the basin.

Total rainfall at Big Spring was 24.08 inches. The greatest monthly rainfall was in September. Calcium and sulfate were the predominant ions in the rain and the median ammonia and nitrate concentrations as nitrogen were 0.40 and $0.37 \mathrm{mg} / \mathrm{L}$ (milligrams per liter), respectively.

Stream discharge, water temperature, specific conductance, and $\mathrm{pH}$ were monitored continuously and monthly water-quality samples were collected at three sites in the basin. In the streams, water temperature and $\mathrm{pH}$ vary diurnally and are greatest during the day. Specific conductance varies inversely with water temperature and $\mathrm{pH}$. The predominant ions in the streams were calcium, magnesium, and bicarbonate. Nitrate plus nitrite as nitrogen concentrations ranged from 0.5 to $15 \mathrm{mg} / \mathrm{L}$. Pesticide concentrations ranged from less than $0.10 \mu \mathrm{g} / \mathrm{L}$ (micrograms per 1 iter) to $0.72 \mu \mathrm{g} / \mathrm{L}$. Atrazine was detected in 12 of 13 stream samples and cyanazine was detected in 4 of 13 samples.

The daily mean temperature of the water in Big Spring ranged from 9.7 to 10.6 degrees Celsius, the daily mean specific conductance ranged from 698 to 735 microsiemens per centimeter at 25 degrees Celsius, and the daily median $p H$ ranged from 6.7 to 7.1 . Calcium, magnesium, and bicarbonate were the predominant ions in solution. Nitrate plus nitrite as nitrogen concentrations ranged from 7.5 to $11 \mathrm{mg} / \mathrm{L}$. Atrazine was the only pesticide detected in the monthly samples from Big Spring. Atrazine concentrations were greater than the detection limit in six of seven samples and ranged from less than 0.10 to $0.26 \mu \mathrm{g} / \mathrm{L}$.

During a baseflow seepage study, June 28 and 29, the discharge lost by streams in the basin was 5.57 cubic feet per second and the dissolved nitrogen load lost was 0.19 tons per day. The discharge and total dissolved nitrogen leaving the basin in streams was 2.93 cubic feet per second and 0.02 tons per day, respectively. 


\section{INTRODUCTION}

There is a concern nationally, as we11 as within the State of Iowa, to understand, quantify, and minimize the occurrence of agricultural chemicals in surface water and ground water. In response to this concern, the Big Spring ground water basin in northeast Iowa, has been studied since 1980 and has become a nationally known demonstration area for improving ground-water quality through the modification of agricultural practices. Numerous multidisciplinary studies that deal with agronomy, geology, hydrology, biology, and socioeconomics of the basin are currently being conducted.

The unique ground-water-flow system within the Big Spring basin aids in studying the movement of agricultural chemicals in the ground water. Much of the ground water in the basin moves through a karst drainage system and is discharged at Big Spring. The extent of the ground-water drainage basin has been defined by dye tracing, potentiometric-surface mapping, and other hydrologic analyses. Nearly all land in the basin is farmed and a clear link between agricultural chemicals and ground-water contamination has been established (Ha11berg and others, 1983, 1984 Libra and others, 1986).

Beginning in October 1987, the U.S. Geological Survey in cooperation with the Iowa Department of Natural Resources, Geological Survey Bureau began a study to collect water quality and quantity data in the Big Spring basin. These data are needed for a detailed understanding of the hydrologic cycle within the basin and for an understanding of agricultural chemical transport processes in the system. The dynamic nature of the surface water and ground-water flow within the basin requires that some facets of water quality and quantity be continuously monitored. Data collected in the study also will aid in interpreting the nature of the flow system. This work also includes determining a partial water budget for the ground-water basin by measuring the input (precipitation) into the basin and the outputs (stream discharge and spring discharge) from the basin.

In addition to the data presented in this report, the Iowa Department of Natural Resources, Geological Survey Bureau and other state and university investigators collected water-quality and discharge data in the study area. These data will be published in separate reports.

\section{Purpose and Scope}

The purpose of this report is to present the hydrologic data collected in the Big Spring basin in the 1988 water year, October 1987 to September 1988. These data include information on the quantity of precipitation and the quantity and quality of ground water, and surface water. Precipitation quality data from the NADP/NTN (National Atmospheric Deposition Program/ National Trends Network) site at Big Spring also are included. 
The data are presented in two formats to aid the reader.

Data recorded continuously are presented graphically and are summarized in tables showing mean daily values. The results of chemical analyses of samples collected monthly at the monitoring sites and of samples collected during the seepage run are listed in tables.

\section{$\underline{\text { Study area }}$}

The study area is located in Clayton County in northeastern Iowa and corresponds with the ground-water basin draining through Big Spring (Hallberg and others, 1983). The basin includes $103 \mathrm{mi}^{2}$ (square miles). One monitoring site (RC2) is located on Roberts Creek above Saint 0laf, Iowa (fig. 1). Roberts Creek and its major tributary, Silver Creek drain approximately 69 percent ( $70.7 \mathrm{mi}^{2}$ ) of the study area overlying the ground-water basin. The remaining area is drained by Howard (approximately $18 \mathrm{mi}^{2}$ ) and Hatchery Creeks (8.8 $\mathrm{mi}^{2}$ ) and several small intermittent streams. A monitoring site (BOOGD) is located on a small stream, Unnamed Creek near Luana, Iowa, that drains into a series of sinkholes. Station numbers for stream sites were the same as those assigned by the Iowa Department of Natural Resources, Geological Survey Bureau in previous studies (Hallberg and others, 1984). A stream-gaging site (L23) is located on Silver Creek. Additional sampling sites for a seepage study are given in table 1. Ground-water discharge is monitored at Big Spring.

\section{Methodology}

Precipitation is measured using standard rain gages at three sites. At two sites, RC2 and BOOGD, precipitation is recorded digitally by a datalogger every 15 minutes while at Big Spring it is recorded continuously on analog chart.

Precipitation quality samples obtained at Big Spring are collected automatically with an Aerochem Metrics Wet/Dry Precipitation Collector. 1/ During periods of rainfall, a bucket is exposed to catch the rain. Between rain events the bucket is covered to avoid the collection of particulate matter. Buckets are removed and the contents analyzed weekly. Site operations are described in detail in the NADP/NTN instruction manual (NADP/NTN, 1988).

1/ Use of trade names in this report is for identification purposes only and does not constitute endorsement by the U.S. Geological Survey 


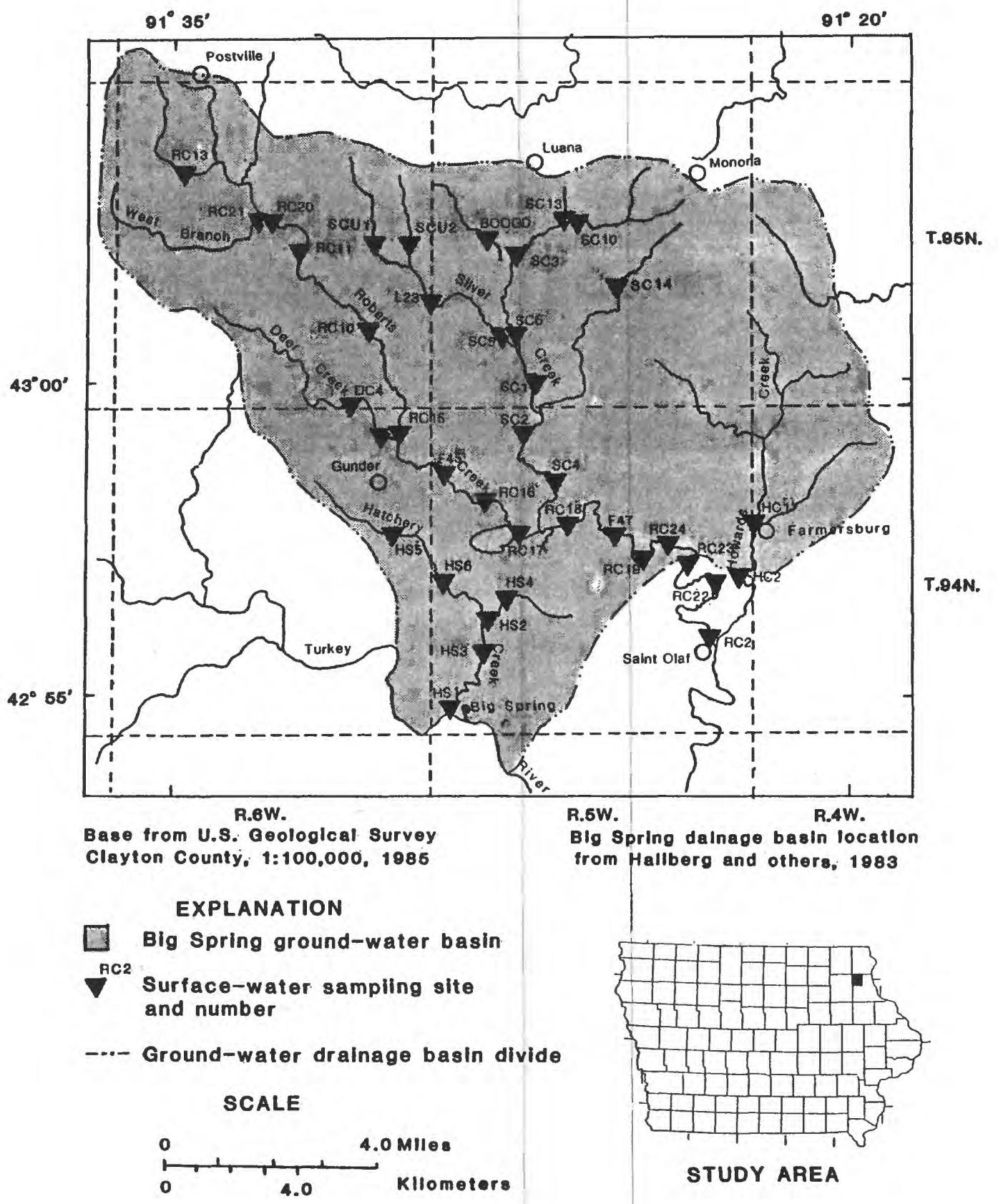

Figure 1.--Location of the study area and sampling sites. 
Table 1.--Location and drainage area of sampling sites

[nr, near; SE, southeast; N, north; SW, southwest; NW, northwest;

$W$, west; E, east; NE, northeast; S, south; trib, tributary; Cr, Creek]

\begin{tabular}{|c|c|c|c|c|c|}
\hline \multirow{2}{*}{$\begin{array}{l}\text { Station } \\
\text { number }\end{array}$} & \multirow{2}{*}{$\begin{array}{l}\text { Site } \\
\text { identi- } \\
\text { cation } \\
\text { number }\end{array}$} & \multirow[b]{2}{*}{ Station name } & \multicolumn{2}{|c|}{ Location } & \multirow{2}{*}{$\begin{array}{l}\text { Drainage } \\
\text { area(mi2 }\end{array}$} \\
\hline & & & Lat. & Long. & \\
\hline HS5 & & Hatchery Creek nr Gunder & 425734 & 0913012 & 1.28 \\
\hline HS6 & & Hatchery Creek SE of Gunder & 425647 & 0912859 & 2.84 \\
\hline HS4 & & Hatchery $\mathrm{Cr}$ trib $\mathrm{N}$ of Big Spring & 425629 & 0912737 & 1.36 \\
\hline HS2 & & Hatchery $\mathrm{Cr}$ trib nr Big Spring & 425606 & 0912806 & 1.85 \\
\hline HS 3 & & Hatchery Creek nr Big Spring & 425536 & 0912806 & 7.02 \\
\hline HS1 & & Hatchery Creek at Big Spring & 425446 & 0912853 & 8.80 \\
\hline $\mathrm{RC} 13$ & & Roberts Creek trib nr Postville & 430327 & 0913440 & 2.28 \\
\hline $\mathrm{RC} 21$ & & West Branch Roberts $\mathrm{Cr}$ at mouth & 430244 & 0913300 & 4.14 \\
\hline RC20 & & Roberts Creek nr Postville & 430240 & 0913253 & 11.1 \\
\hline $\mathrm{RC11}$ & & Roberts Creek SE of Postville & 430211 & 0913216 & 13.2 \\
\hline $\mathrm{RC10}$ & & Roberts Creek nr Luana & 430057 & 0913042 & 15.9 \\
\hline RC15 & & Roberts Creek at Gunder & 425908 & 0913002 & 18.2 \\
\hline DC4 & & Deer Creek nr Gunder & 425942 & 0913107 & 4.37 \\
\hline DC2 & & Deer Creek at Gunder & 425908 & 0913025 & 5.56 \\
\hline F45 & & Roberts Creek East of Gunder & 425830 & 0912858 & 26.0 \\
\hline $\mathrm{RC} 16$ & & Roberts Creek north of Big Spring & 425806 & 0912805 & 28.8 \\
\hline RC17 & & Roberts Creek near Big Spring & 425735 & 0912722 & 30.4 \\
\hline SC10 & & East Fork Silver $\mathrm{Cr} \mathrm{nr}$ Monona & 430240 & 0912620 & 3.05 \\
\hline $\mathrm{SC} 13$ & & E Fork Silver Cr trib nr Monona & 430240 & 0912606 & .28 \\
\hline SC3 & & E Fork Silver Cr nr Luana & 430203 & 0912730 & 4.28 \\
\hline BOOGD & 05412070 & Unnamed Creek near Luana & 430224 & 0912807 & 1.15 \\
\hline SC6 & & E Fork Silver Creek $S$ of Luana & 430054 & 0912730 & 9.46 \\
\hline SCU1 & & Silver Creek SW of Luana & 430210 & 0913033 & 1.36 \\
\hline SCU2 & & Silver Creek trib SW of Luana & 430201 & 0912949 & .70 \\
\hline L23 & 05412060 & Silver Creek nr Luana & 430119 & 0912921 & 4.39 \\
\hline SC5 & & Silver Creek South of Luana & 430049 & 0912744 & 5.59 \\
\hline SC1 & & Silver Creek NE of Gunder & 430002 & 0912653 & 17.3 \\
\hline SC14 & & Silver Creek trib nr Monona & 430140 & 0912510 & 1.13 \\
\hline $\mathrm{SC} 2$ & & Silver Creek nr Gunder & 425916 & 0912712 & 25.2 \\
\hline SC4 & & Silver Creek East of Gunder & 425824 & 0912630 & 28.8 \\
\hline $\mathrm{RC} 18$ & & Roberts Creek NE of Big Spring & 425736 & 0912603 & 61.8 \\
\hline RC19 & & Roberts Creek NW of Saint Olaf & 425733 & 0912510 & 63.6 \\
\hline F47 & & Roberts Creek $W$ of Farmersburg & 425706 & 0912434 & 64.3 \\
\hline RC24 & & Roberts Creek nr Farmersburg & 425724 & 0912358 & 65.2 \\
\hline $\mathrm{RC} 23$ & & Roberts Creek $\mathrm{N}$ of Saint olaf & 425710 & 0912328 & 66.0 \\
\hline RC22 & & Roberts Creek SW of Farmersburg & 425641 & 0912226 & 66.6 \\
\hline $\mathrm{RC} 2$ & 05412100 & Roberts Creek above Saint 0laf & 425549 & 0912303 & 70.7 \\
\hline $\mathrm{HC1}$ & & Howard Creek at Farmersburg & 425744 & 0912209 & 13.8 \\
\hline $\mathrm{HC} 2$ & & Howard Creek nr Farmersburg & 425648 & 0912223 & 17.8 \\
\hline
\end{tabular}


Measurement of unstable constituents and stream discharge are made at the time of sample collection. Water temperatures and dissolved-oxygen concentrations are measured in the stream or spring pool. Water temperatures are measured with a standard mercury or alcohol thermometer that has been previously checked against a laboratory grade thermometer for accuracy. Dissolved-oxygen concentrations are measured with a dissolved-oxygen meter. Water to be analyzed is collected from a flowing section of the stream or the surface of the spring pool. Immediately after sample collection, the pH and specific conductance of the water are measured. Stream discharge normally is measured by current-meter methods (Buchanan and Somers, 1969). Because of low-flow conditions with shallow stream depths during most of 1988 water year, the 0.6 -depth method generally is used. Where the channel is extremely narrow and the discharge is small, less than $0.50 \mathrm{ft}^{3} / \mathrm{s}$ (cubic feet per second), a portable Parshall flume (Kilpatrick and Schneider, 1983, p 13-15) is used to measure the flow. Stage is recorded continuously at stream sites RC2, L23, and BOOGD with bubble-gage sensors and digital recorders (Rantz and others, 1982a, p 32-39). Stream discharge is calculated from stage using stage-discharge relations developed for each site (Kennedy, 1983, p 30-32).

Samples for chemical analyses are prepared as described in table 2 for shipment to the laboratory. Analyses of water samples by the University Hygienics Laboratory in Iowa City and Des Moines, Iowa are by the methods listed in table 2. The U.S. Environmenta1 Protection Agency's method 81.40 for the analyses of pesticides is modified to use capillary columns.

The water-quality constituents continuously monitored at two stream sites and at Big Spring are water temperature, specific conductance, and $\mathrm{pH}$. These constituents are measured using a multiple parameter meter and recorded at 15 minute intervals with a Campbell datalogger. The data stored in the datalogger is retrieved weekly by computer through a phone modem in the U.S. Geological Survey's office in Iowa City. From the 15 minute observations, mean daily values are calculated and permanently stored in the U.S. Geological Survey National Water Data Storage and Retrieval System (WATSTORE) data base. Values determined by the multiple parameter field meter are checked weekly against $\mathrm{pH}$ buffer and conductance reference solutions. Temperature values are checked against a mercury thermometer. Water samples for the analyses of major ions, nutrients, and selected pesticides are collected the first week of each month at the monitoring sites starting in March 1988.

Suspended sediment samples are collected periodically by local observers. The observers collected depth integrated samples at one vertical using techniques described by Guy and Norman, (1970). Samples were collected five times per week at RC2, Roberts Creek above Saint 01af, and three times per week at BOOGD, Unnamed Creek near Luana. 
Table 2,--Sample preparation and chemical analysis procedures [EPA methods from U.S. Environmental Protection Asency, 1983] [USGS1 - Buchanan and Somers, 1969; USGS2 - Wood, 1976] [um, micrometer; C, Carbon; N, nitrogen; P, phosporus]

[*, modified method see text]

\begin{tabular}{|c|c|}
\hline Sample preparation method & Chemical constituent \\
\hline $\begin{array}{l}\text { Filtration through a } 0.45 \mathrm{um} \\
\text { membrane } \\
\text { Acidification with nitric acid } \\
\text { Chill } \\
\text { Acidification with sulfuric acid }\end{array}$ & $\begin{array}{l}\text { Calcium, magnesium, sodium, potassium } \\
\text { sulfate, chloride, dissolved solids, } \\
\text { silica, aluminum, iron, nitrate plus } \\
\text { nitrite, organic nitrogen, ammonia } \\
\text { Calcium, magnesium, sodium, potassium, } \\
\text { iron, aluminum } \\
\text { Organic carbon, nitrate plus nitrite, } \\
\text { ammonia, organic nitrogen, } \\
\text { orthophosphate, atrazine, cyanazine, } \\
\text { metolachlor, alachlor, metribuzin, } \\
\text { butylate, trifluralin } \\
\text { nitrate plus nitrite, ammonia, } \\
\text { orthophosphate }\end{array}$ \\
\hline Constituent & $\begin{array}{l}\text { Analytical } \\
\text { method }\end{array}$ \\
\hline $\begin{array}{l}\text { Field Measurements } \\
\text { Stream discharge } \\
\text { Water temperature } \\
\text { Specific conductance } \\
\text { pH } \\
\text { Oxysen, dissolved } \\
\text { Alkalinity } \\
\text { Bicarbonate, dissolved } \\
\text { Carbonate, dissoved }\end{array}$ & $\begin{array}{l}\text { USGS1 } \\
\text { USGS2 } \\
\text { USGS2 } \\
\text { USGS2 } \\
\text { USGS2 } \\
\text { Incremental titration } \\
\text { Incremental titration } \\
\text { Incremental titration }\end{array}$ \\
\hline $\begin{array}{l}\text { Inorganic compounds, dissolved } \\
\text { Calcium } \\
\text { Magnesium } \\
\text { Sodium } \\
\text { Potassium } \\
\text { Chloride } \\
\text { Sulfate } \\
\text { Silica } \\
\text { Aluninum } \\
\text { Iron }\end{array}$ & $\begin{array}{l}\text { EPA method } 215.2 \\
\text { EPA method } 200.7 \\
\text { EPA method } 273.1 \\
\text { EPA method } 258.1 \\
\text { EPA method } 325.3 \\
\text { EPA method } 275.4 \\
\text { EPA method } 370.1 \\
\text { EPA method } 200.7 \\
\text { EPA method } 200.7\end{array}$ \\
\hline $\begin{array}{l}\text { Nutrients dissolved } \\
\text { Nitrate plus nitrite as } N \\
\text { Ammonia as } N \\
\text { Organic nitrogen as } N \\
\text { Carbon, total organic as } C \\
\text { Orthophosphate as } P\end{array}$ & $\begin{array}{l}\text { EPA method } 353.2 \\
\text { EPA method } 350.1 \\
\text { EPA method } 415.1 \\
\text { EPA method } 415.1 \\
\text { EPA method } 365.1\end{array}$ \\
\hline $\begin{array}{l}\text { Pesticides, total recoverable } \\
\text { Atrazine } \\
\text { Cyanazine } \\
\text { Metolachlor } \\
\text { Alachlor } \\
\text { Metribuzin } \\
\text { Butylate } \\
\text { Trifluralin }\end{array}$ & 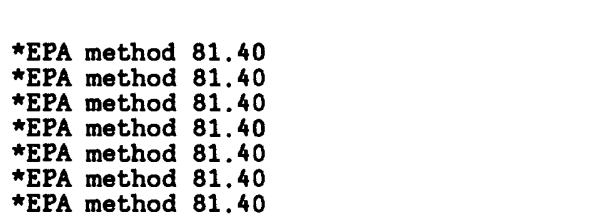 \\
\hline
\end{tabular}




\section{Acknowledgments}

This project was supported, in part, by the Iowa Department of Natural Resources Geological Survey Bureau, through the Big Spring Basin Demonstration Project, with funds provided from the Iowa Groundwater Protection Act. George Hallberg, Coordinator of the Big Spring Basin Demonstration Project, Bob Libra, John Littke, and Deb Quade of the Iowa Department of Natural Resources, Geological Survey Bureau, provided technical advice and field support. Roger Koster, the Big Spring Project Coordinator for the Iowa State University Cooperative Extension Service, located Clayton County residents willing to serve as local observers to collect periodic sediment samples. These residents are, Sarah Hilgerson, Karen and Eugene Voss, and Jerry Koonze.

\section{HYDROLOGIC DATA}

\section{Precipitation}

\section{Quantity}

Measurable precipitation of 0.01 in. (inch) or more fell 36 of 52 weeks during water year 1988 at Big Spring. Total rainfall for water year 1988 was 24.08 in. with a median weekly rainfall of 0.22 in. (table 3 ). The total rainfall at Big Spring was 9.25 in. less than the average for the nearby National Weather Service station in Elkader, Iowa (National Oceanic and Atmospheric Administration, 1988, p 3.). The greatest weekly rainfall (3.68 in.) occurred from September 18 to 24.

Precipitation was measured at site BOOGD from May 14 to the end of the water year and at site RC2 from June 1 to the end of the water year (table 4). During this period, the greatest monthly rainfall occurred in September when 4.89 and 4.82 in. fell at site $B O O G D$ and $R C 2$, respectively. The greatest daily rainfall at site BOOGD (2.37 in.) fell on September 19 and the greatest daily rainfall at site RC2 (2.26 in.) fell on september 22.

\section{Quality}

Weekly precipitation samples were collected for 33 of 36 weeks of measurable rainfall that occurred at Big Spring. Results of chemical analyses of these samples are summarized in table 3. The median concentration of the predominant cation, calcium, was $0.81 \mathrm{mg} / \mathrm{L}$ and the median concentration of the predominant anion, sulfate, was $2.0 \mathrm{mg} / \mathrm{L}$. The median concentrations of nitrate and ammonia, were 0.40 an $0.37 \mathrm{mg} / \mathrm{L}$ as nitrogen, respectively. Maximum concentrations of all major ions were less than $10 \mathrm{mg} / \mathrm{L}$. 
Table 3.--Statistical summary of precipitation quantity and quality at Big Spring water year October 1987 to September 1988

[Chemical constituents in milligrams per 1iter]

[ $\mu \mathrm{S} / \mathrm{cm}$, microsiemens per centimeter at 25 degrees Celsius; $N$, nitrogen]

\begin{tabular}{lcccc}
\hline & $\begin{array}{l}\text { Number of } \\
\text { weekly } \\
\text { samples }\end{array}$ & Median & Minimum & Maximum \\
Constituent & 52 & .22 & 0.0 & 3.68 \\
Precipitation (inches) & 33 & 5.81 & 4.19 & 7.41 \\
Lab pH (units) & 33 & 17 & 2.6 & 60 \\
Lab conductance ( $\mu \mathrm{S} / \mathrm{cm})$ & 33 & .81 & .07 & 4.4 \\
Calcium & 33 & .10 & .02 & .52 \\
Magnesium & 33 & .12 & .02 & 4.5 \\
& 33 & .04 & $<.01$ & .70 \\
Sodium & 33 & 2.0 & .03 & 9.2 \\
Potassium & 32 & .16 & .05 & 1.1 \\
Sulfate & 33 & .40 & $<.01$ & 1.8 \\
Chloride & 33 & .37 & $<.02$ & 1.8 \\
Nitrate as N & & & & \\
Ammonia as N & & & \\
\hline
\end{tabular}


Table 4.--Accumulated daily rainfall, water year October 1987 to September 1988

Day Oct Nov Dec Jan Feb Mar Apr May June July Aug Sept

BOOGD, Unnamed Creek at Luana

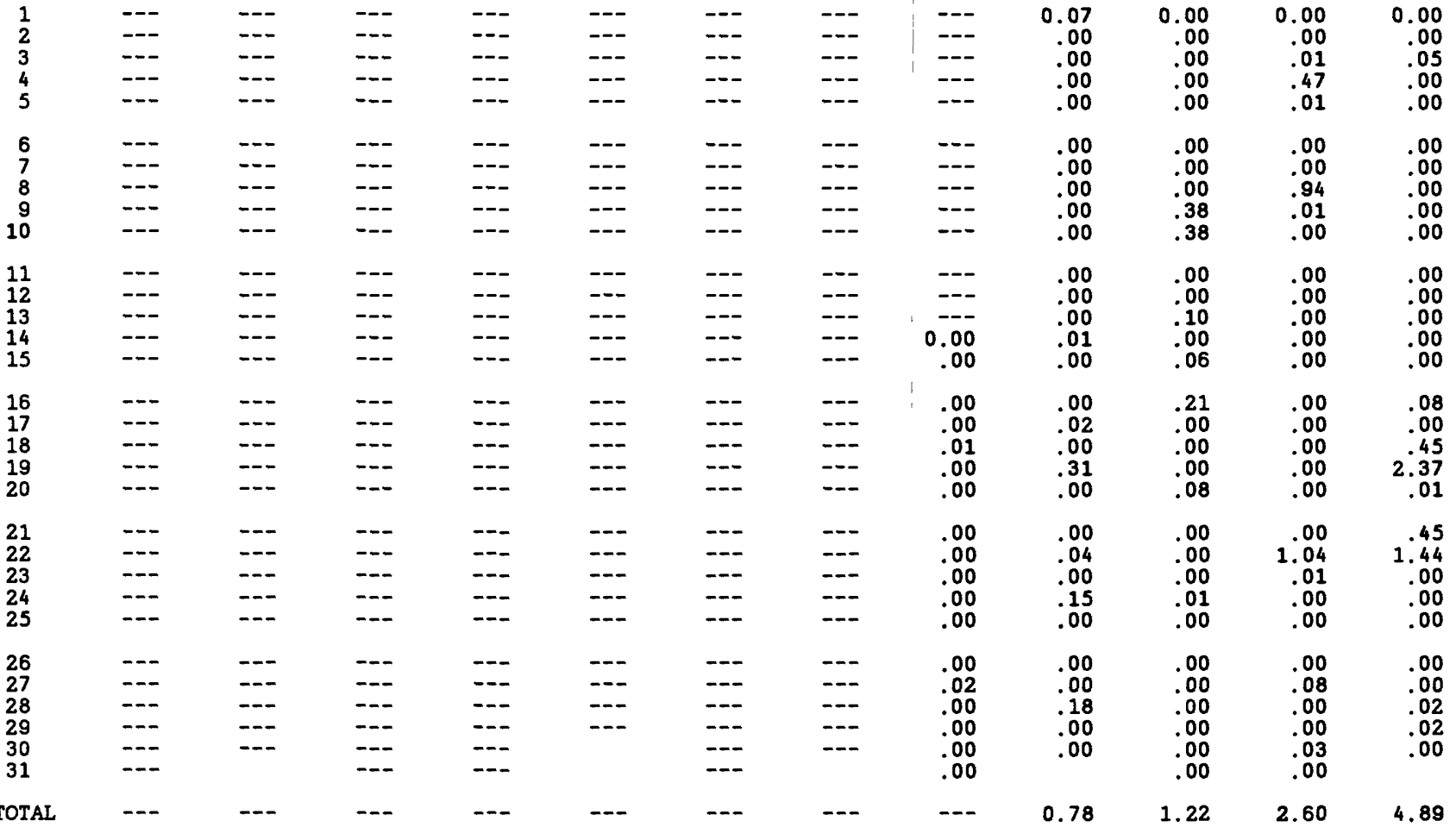

RC2, Roberts Creek above Saint Olaf

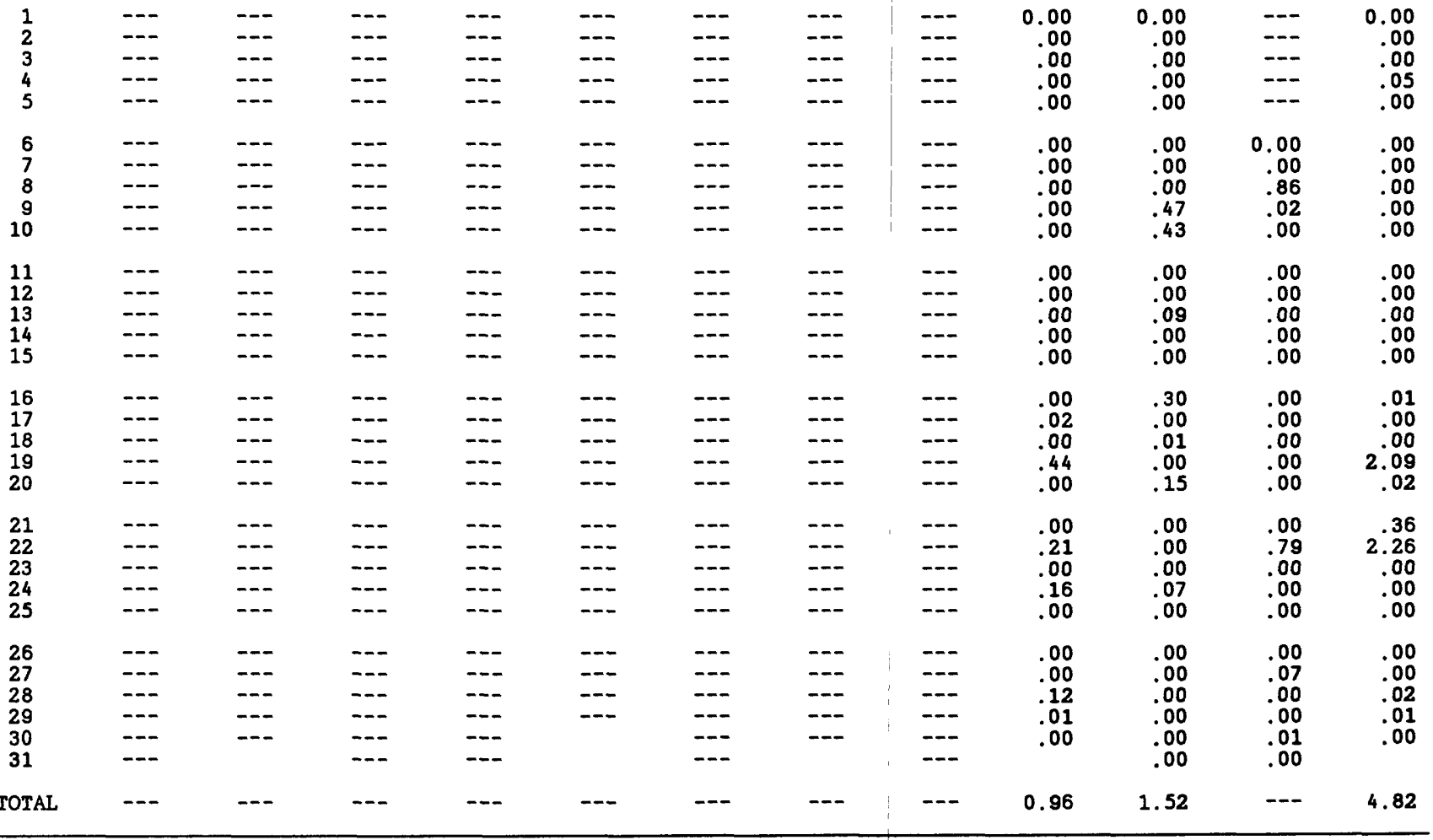




\section{Surface water}

\section{Silver Creek}

The drainage area upstream of site L23, Silver Creek near Luana, is 3.76 $\mathrm{mi}^{2}$. Daily mean discharge at this site ranged from $0.05 \mathrm{ft}^{3} / \mathrm{s}$ on September 14 and 15 to $26 \mathrm{ft}^{3} / \mathrm{s}$ on March 1 during snowmelt (table 5). Mean discharge for the 1988 water year was $1.59 \mathrm{ft}^{3} / \mathrm{s}$.

\section{Discharge}

\section{Unnamed Creek}

The daily mean discharge at site BOOGD, Unnamed Creek near Luana, ranged from 0.0 to $7.0 \mathrm{ft}^{3} / \mathrm{s}$ (table 6 ). The mean and median discharge for the 1988 water year were 0.21 and $0.02 \mathrm{ft}^{3} / \mathrm{s}$, respectively. Unnamed Creek was dry in late December and most of January. The maximum discharge occurred in March during snowmelt. Streamflow again ceased during the last week of June and remained dry through the end of the water year.

\section{Water quality}

A limited amount of water-quality data was collected at Unnamed Creek near Luana because of the lack of streamflow due to the precipitation deficit in water year 1988. Water temperature, specific conductance, and $\mathrm{pH}$ were monitored continuously from the time of instrument installation (May 14) until the stream dried up on June 27. During this period, specific conductance ranged from $687 \mu \mathrm{S} / \mathrm{cm}$ (microsiemens per centimeter at 25 degrees Celsius) on May 27 to $819 \mu \mathrm{S} / \mathrm{cm}$ on June 20 (table 6). Overall, daily mean specific conductance increased during the month of June (fig. 2) and the daily the daily mean water temperature gradually increased. The minimum mean daily temperature was $10.5{ }^{\circ} \mathrm{C}$ (degrees Celsius) on May 16 and the maximum daily mean temperature was $23.8^{\circ} \mathrm{C}$ on June 20 . During this same period, the median $\mathrm{pH}$ declined. The maximum daily median $\mathrm{pH}$ was 8.21 units on May 15 and the minimum was 7.59 units on June 20 (table 7). Field measurements of instantaneous discharge, specific conductance, $\mathrm{pH}$, water temperature, and dissolved oxygen collected March to September at monthly monitoring sites are given in table 8.

Chemical analyses of the dissolved constituents in three monthly samples show that calcium and magnesium were the dominant cations in solution (table 9 ). Concentrations of calcium ranged from 44 to $79 \mathrm{mg} / \mathrm{L}$ and concentrations of magnesium ranged from 19 to $33 \mathrm{mg} / \mathrm{L}$. Concentrations of sodium and potassium were less than $10 \mathrm{mg} / \mathrm{L}$. The dominant anion in solution was bicarbonate. Bicarbonate concentrations ranged from 182 to $366 \mathrm{mg} / \mathrm{L}$. Other major anions, sulfate and chloride, were present in concentrations ranging from 12 to 32 $\mathrm{mg} / \mathrm{L}$. Silica concentrations ranged from 10 to $18 \mathrm{mg} / \mathrm{L}$. Nitrate plus nitrite is the dominant nitrogen species in four samples collected from Unnamed Creek near Luana (table 10). Nitrate plus nitrite concentrations ranged from 7.6 to $14 \mathrm{mg} / \mathrm{L}$ as nitrogen. Concentrations in three of the four samples exceed the U.S. Environmental Protection Agency (1986) maximum contaminant level of 10 $\mathrm{mg} / \mathrm{L}$ nitrate-nitrogen for drinking water. Ammonia nitrogen concentrations ranged from less than the detection leve1 to $0.5 \mathrm{mg} / \mathrm{L}$ and organic nitrogen concentrations ranged from 0.1 to $0.8 \mathrm{mg} / \mathrm{L}$. 
Table 5.--Daily mean discharge at site L23, Silver Creek near Luana, Iowa

Water year October 1987 to September 1988

\begin{tabular}{|c|c|c|c|c|c|c|c|c|c|c|c|c|}
\hline Day & Oct & Nov & Dec & Jan & Feb & Mar & Apr & May & June & July & Aug & Sept \\
\hline & \multicolumn{12}{|c|}{ Daily mean discharge, in cubic feet, per second } \\
\hline $\begin{array}{l}1 \\
2 \\
3 \\
4 \\
5\end{array}$ & $\begin{array}{l}1.6 \\
1.5 \\
1.5 \\
1.4 \\
1.4\end{array}$ & $\begin{array}{l}1.1 \\
1.4 \\
1.3 \\
1.1 \\
1.1\end{array}$ & $\begin{array}{r}.88 \\
.96 \\
1.0 \\
.70 \\
.64\end{array}$ & $\begin{array}{l}.72 \\
.70 \\
.68 \\
.58 \\
.49\end{array}$ & $\begin{array}{r}2.4 \\
2.0 \\
2.0 \\
1.2 \\
.90\end{array}$ & $\begin{array}{c}26 \\
17 \\
7.4 \\
6.0 \\
7.1\end{array}$ & $\begin{array}{l}3.5 \\
5.5 \\
7.3 \\
5.8 \\
5.5\end{array}$ & $\begin{array}{l}1.2 \\
1.1 \\
1.0 \\
.97 \\
.94\end{array}$ & $\begin{array}{l}1.0 \\
1.0 \\
.89 \\
1.1 \\
1.0\end{array}$ & $\begin{array}{l}.58 \\
.66 \\
.66 \\
.58 \\
.60\end{array}$ & $\begin{array}{l}.24 \\
.22 \\
.25 \\
.31 \\
.31\end{array}$ & $\begin{array}{l}.12 \\
.11 \\
.20 \\
.16 \\
.12\end{array}$ \\
\hline $\begin{array}{r}6 \\
7 \\
8 \\
9 \\
10\end{array}$ & $\begin{array}{l}1.3 \\
1.3 \\
1.3 \\
1.2 \\
1.2\end{array}$ & $\begin{array}{l}1.0 \\
1.0 \\
1.8 \\
1.6 \\
1.4\end{array}$ & $\begin{array}{r}.78 \\
.97 \\
1.2 \\
1.9 \\
1.6\end{array}$ & $\begin{array}{l}.43 \\
.48 \\
.52 \\
.49 \\
.45\end{array}$ & $\begin{array}{l}.76 \\
.68 \\
.63 \\
.58 \\
.56\end{array}$ & $\begin{array}{l}17 \\
13 \\
19 \\
9.1 \\
7.6\end{array}$ & $\begin{array}{l}5.1 \\
4.4 \\
3.9 \\
3.4 \\
3.2\end{array}$ & $\begin{array}{l}.90 \\
.88 \\
5.4 \\
5.3 \\
4.3\end{array}$ & $\begin{array}{l}.95 \\
.99 \\
.98 \\
.95 \\
.97\end{array}$ & $\begin{array}{l}.60 \\
.59 \\
.57 \\
.59 \\
.65\end{array}$ & $\begin{array}{l}.24 \\
.21 \\
.33 \\
.26 \\
.21\end{array}$ & $\begin{array}{l}.10 \\
.09 \\
.08 \\
.08 \\
.07\end{array}$ \\
\hline $\begin{array}{l}11 \\
12 \\
13 \\
14 \\
15\end{array}$ & $\begin{array}{l}1.2 \\
1.1 \\
1.2 \\
1.1 \\
1.1\end{array}$ & $\begin{array}{l}1.3 \\
1.8 \\
1.6 \\
1.4 \\
1.3\end{array}$ & $\begin{array}{l}1.6 \\
1.4 \\
1.0 \\
.80 \\
.72\end{array}$ & $\begin{array}{l}.47 \\
.58 \\
.52 \\
.48 \\
.54\end{array}$ & $\begin{array}{l}.55 \\
.54 \\
.53 \\
.58 \\
.56\end{array}$ & $\begin{array}{l}6.9 \\
6.1 \\
5.1 \\
4.9 \\
4.7\end{array}$ & $\begin{array}{l}2.7 \\
2.7 \\
2.5 \\
2.1 \\
1.9\end{array}$ & $\begin{array}{l}3.5 \\
3.1 \\
2.3 \\
2.2 \\
2.0\end{array}$ & $\begin{array}{l}.95 \\
.97 \\
.96 \\
.88 \\
.88\end{array}$ & $\begin{array}{l}.52 \\
.53 \\
.52 \\
.55 \\
.58\end{array}$ & $\begin{array}{l}.22 \\
.17 \\
.15 \\
.13 \\
.13\end{array}$ & $\begin{array}{l}.07 \\
.06 \\
.06 \\
.05 \\
.05\end{array}$ \\
\hline $\begin{array}{l}16 \\
17 \\
18 \\
19 \\
20\end{array}$ & $\begin{array}{l}1.3 \\
1.5 \\
1.3 \\
1.2 \\
1.1\end{array}$ & $\begin{array}{l}1.2 \\
2.0 \\
1.2 \\
1.2 \\
1.1\end{array}$ & $\begin{array}{l}1.6 \\
1.4 \\
1.2 \\
1.3 \\
1.5\end{array}$ & $\begin{array}{l}.68 \\
.64 \\
.56 \\
.51 \\
.48\end{array}$ & $\begin{array}{l}.56 \\
.64 \\
.62 \\
.60 \\
.56\end{array}$ & $\begin{array}{l}3.8 \\
3.6 \\
3.6 \\
3.3 \\
2.6\end{array}$ & $\begin{array}{l}1.8 \\
2.0 \\
1.8 \\
1.8 \\
1.9\end{array}$ & $\begin{array}{l}1.7 \\
1.5 \\
1.7 \\
1.5 \\
1.3\end{array}$ & $\begin{array}{l}.85 \\
.91 \\
.86 \\
.89 \\
.88\end{array}$ & $\begin{array}{l}.58 \\
.52 \\
.53 \\
.48 \\
.44\end{array}$ & $\begin{array}{l}.12 \\
.13 \\
.10 \\
.09 \\
.10\end{array}$ & $\begin{array}{l}.10 \\
.09 \\
.20 \\
.99 \\
.56\end{array}$ \\
\hline $\begin{array}{l}21 \\
22 \\
23 \\
24 \\
25\end{array}$ & $\begin{array}{l}1.1 \\
1.0 \\
.96 \\
.94 \\
.89\end{array}$ & $\begin{array}{l}1.0 \\
1.1 \\
.93 \\
.76 \\
.76\end{array}$ & $\begin{array}{l}.90 \\
1.0 \\
.95 \\
.84 \\
.75\end{array}$ & $\begin{array}{l}.45 \\
.44 \\
.44 \\
.45 \\
.44\end{array}$ & $\begin{array}{l}.54 \\
.58 \\
.58 \\
.55 \\
.53\end{array}$ & $\begin{array}{l}2.5 \\
2.8 \\
2.9 \\
3.5 \\
5.4\end{array}$ & $\begin{array}{l}1.5 \\
1.4 \\
1.6 \\
1.4 \\
1.5\end{array}$ & $\begin{array}{l}1.2 \\
1.1 \\
1.0 \\
.96 \\
.94\end{array}$ & $\begin{array}{l}.72 \\
.73 \\
.69 \\
.78 \\
.70\end{array}$ & $\begin{array}{l}.41 \\
.40 \\
.40 \\
.38 \\
.37\end{array}$ & $\begin{array}{l}.13 \\
.27 \\
.23 \\
.11 \\
.10\end{array}$ & $\begin{array}{r}.32 \\
1.7 \\
.56 \\
.33 \\
.31\end{array}$ \\
\hline $\begin{array}{l}26 \\
27 \\
28 \\
29 \\
30 \\
31\end{array}$ & $\begin{array}{l}.88 \\
3.0 \\
2.0 \\
1.2 \\
1.0 \\
.93\end{array}$ & $\begin{array}{l}.75 \\
.73 \\
1.2 \\
1.2 \\
1.1\end{array}$ & $\begin{array}{l}.70 \\
.68 \\
.79 \\
.76 \\
.80 \\
.80\end{array}$ & $\begin{array}{l}.43 \\
.42 \\
.54 \\
.68 \\
2.0 \\
6.0\end{array}$ & $\begin{array}{l}1.68 \\
3.2 \\
14\end{array}$ & $\begin{array}{l}4.2 \\
3.3 \\
5.0 \\
5.6 \\
5.1 \\
4.0\end{array}$ & $\begin{array}{l}1.8 \\
2.2 \\
1.7 \\
1.5 \\
1.3\end{array}$ & $\begin{array}{l}.94 \\
1.2 \\
1.1 \\
1.1 \\
1.0 \\
1.0\end{array}$ & $\begin{array}{l}.59 \\
.58 \\
.57 \\
.57 \\
.57\end{array}$ & $\begin{array}{l}.35 \\
.36 \\
.31 \\
.33 \\
.28 \\
.28\end{array}$ & $\begin{array}{l}.09 \\
.18 \\
.15 \\
.13 \\
.15 \\
.14\end{array}$ & $\begin{array}{l}.28 \\
.22 \\
.17 \\
.19 \\
.21\end{array}$ \\
\hline
\end{tabular}


Table 6.--Daily mean discharge and specific conductance at site BOOGD,

Unnamed Creek near Luana Iowa

Water year October 1987 to September 1988

[---, data not available to calculate mean values]

\begin{tabular}{|c|c|c|c|c|c|c|c|c|c|c|c|c|}
\hline Day & Oct & Nov & Dec & Jan & Feb & Mar & Apr & May & $\operatorname{Jun} e$ & July & Aus & Sept \\
\hline & \multicolumn{12}{|c|}{ Daily mean discharge, in cubic feet per second } \\
\hline $\begin{array}{l}1 \\
2 \\
3 \\
4 \\
5\end{array}$ & $\begin{array}{l}.19 \\
.17 \\
.16 \\
.15 \\
.14\end{array}$ & $\begin{array}{l}.04 \\
.03 \\
.03 \\
.02 \\
.02\end{array}$ & $\begin{array}{l}.04 \\
.03 \\
.02 \\
.01 \\
.01\end{array}$ & $\begin{array}{l}.00 \\
.00 \\
.00 \\
.00 \\
.00\end{array}$ & $\begin{array}{r}.11 \\
.45 \\
.53 \\
.21 \\
.10\end{array}$ & $\begin{array}{c}7.0 \\
3.5 \\
.80 \\
.40 \\
1.0\end{array}$ & $\begin{array}{l}.66 \\
.93 \\
2.6 \\
2.3 \\
1.8\end{array}$ & $\begin{array}{l}.14 \\
.18 \\
.16 \\
.15 \\
.15\end{array}$ & $\begin{array}{l}.07 \\
.06 \\
.06 \\
.05 \\
.05\end{array}$ & $\begin{array}{l}.00 \\
.00 \\
.00 \\
.00 \\
.00\end{array}$ & $\begin{array}{l}.00 \\
.00 \\
.00 \\
.00 \\
.00\end{array}$ & $\begin{array}{l}.00 \\
.00 \\
.00 \\
.00 \\
.00\end{array}$ \\
\hline $\begin{array}{r}6 \\
7 \\
8 \\
9 \\
10\end{array}$ & $\begin{array}{l}.13 \\
.12 \\
.11 \\
.10 \\
.09\end{array}$ & $\begin{array}{l}.02 \\
.02 \\
.04 \\
.03 \\
.02\end{array}$ & $\begin{array}{l}.01 \\
.02 \\
.04 \\
.06 \\
.03\end{array}$ & $\begin{array}{l}.00 \\
.00 \\
.01 \\
.01 \\
.01\end{array}$ & $\begin{array}{l}.05 \\
.03 \\
.02 \\
.02 \\
.01\end{array}$ & $\begin{array}{l}2.5 \\
6.0 \\
4.0 \\
2.8 \\
2.5\end{array}$ & $\begin{array}{c}1.3 \\
1.1 \\
.85 \\
1.1 \\
.95\end{array}$ & $\begin{array}{l}.14 \\
.13 \\
.35 \\
.20 \\
.13\end{array}$ & $\begin{array}{l}.05 \\
.06 \\
.06 \\
.06 \\
.06\end{array}$ & $\begin{array}{l}.00 \\
.00 \\
.00 \\
.00 \\
.00\end{array}$ & $\begin{array}{l}.00 \\
.00 \\
.00 \\
.00 \\
.00\end{array}$ & $\begin{array}{l}.00 \\
.00 \\
.00 \\
.00 \\
.00\end{array}$ \\
\hline $\begin{array}{l}11 \\
12 \\
13 \\
14 \\
15\end{array}$ & $\begin{array}{l}.09 \\
.09 \\
.08 \\
.08 \\
.07\end{array}$ & $\begin{array}{l}.02 \\
.03 \\
.03 \\
.02 \\
.02\end{array}$ & $\begin{array}{l}.04 \\
.04 \\
.03 \\
.02 \\
.01\end{array}$ & $\begin{array}{l}.00 \\
.00 \\
.00 \\
.01 \\
.01\end{array}$ & $\begin{array}{l}.01 \\
.01 \\
.01 \\
.01 \\
.01\end{array}$ & $\begin{array}{l}2.1 \\
1.9 \\
1.2 \\
.98 \\
.78\end{array}$ & $\begin{array}{l}.75 \\
.81 \\
.78 \\
.65 \\
.54\end{array}$ & $\begin{array}{l}.10 \\
.12 \\
.12 \\
.13 \\
.12\end{array}$ & $\begin{array}{l}.06 \\
.05 \\
.05 \\
.06 \\
.06\end{array}$ & $\begin{array}{l}.00 \\
.00 \\
.00 \\
.00 \\
.00\end{array}$ & $\begin{array}{l}.00 \\
.00 \\
.00 \\
.00 \\
.00\end{array}$ & $\begin{array}{l}.00 \\
.00 \\
.00 \\
.00 \\
.00\end{array}$ \\
\hline $\begin{array}{l}16 \\
17 \\
18 \\
19 \\
20\end{array}$ & $\begin{array}{l}.15 \\
.13 \\
.10 \\
.08 \\
.06\end{array}$ & $\begin{array}{l}.02 \\
.06 \\
.02 \\
.01 \\
.02\end{array}$ & $\begin{array}{l}.01 \\
.01 \\
.01 \\
.01 \\
.01\end{array}$ & $\begin{array}{l}.00 \\
.00 \\
.00 \\
.00 \\
.00\end{array}$ & $\begin{array}{l}.01 \\
.01 \\
.02 \\
.01 \\
.01\end{array}$ & $\begin{array}{l}.67 \\
.60 \\
.46 \\
.42 \\
.29\end{array}$ & $\begin{array}{l}.51 \\
.53 \\
.51 \\
.40 \\
.40\end{array}$ & $\begin{array}{l}.13 \\
.11 \\
.12 \\
.22 \\
.21\end{array}$ & $\begin{array}{l}.06 \\
.06 \\
.05 \\
.05 \\
.04\end{array}$ & $\begin{array}{l}.00 \\
.00 \\
.00 \\
.00 \\
.00\end{array}$ & $\begin{array}{l}.00 \\
.00 \\
.00 \\
.00 \\
.00\end{array}$ & $\begin{array}{l}.00 \\
.00 \\
.00 \\
.00 \\
.00\end{array}$ \\
\hline $\begin{array}{l}21 \\
22 \\
23 \\
24 \\
25\end{array}$ & $\begin{array}{l}.05 \\
.05 \\
.04 \\
.04 \\
.03\end{array}$ & $\begin{array}{l}.01 \\
.02 \\
.02 \\
.02 \\
.03\end{array}$ & $\begin{array}{l}.01 \\
.01 \\
.01 \\
.01 \\
.01\end{array}$ & $\begin{array}{l}.00 \\
.00 \\
.00 \\
.00 \\
.00\end{array}$ & $\begin{array}{l}.01 \\
.02 \\
.02 \\
.01 \\
.01\end{array}$ & $\begin{array}{r}.25 \\
.31 \\
.32 \\
.91 \\
.77\end{array}$ & $\begin{array}{l}.30 \\
.29 \\
.26 \\
.25 \\
.24\end{array}$ & $\begin{array}{l}.21 \\
.20 \\
.14 \\
.08 \\
.07\end{array}$ & $\begin{array}{l}.04 \\
.04 \\
.04 \\
.04 \\
.02\end{array}$ & $\begin{array}{l}.00 \\
.00 \\
.00 \\
.00 \\
.00\end{array}$ & $\begin{array}{l}.00 \\
.00 \\
.00 \\
.00 \\
.00\end{array}$ & $\begin{array}{l}.00 \\
.00 \\
.00 \\
.00 \\
.00\end{array}$ \\
\hline $\begin{array}{l}26 \\
27 \\
28 \\
29 \\
30 \\
31\end{array}$ & $\begin{array}{l}.03 \\
.04 \\
.04 \\
.03 \\
.03 \\
.02\end{array}$ & $\begin{array}{l}.04 \\
.04 \\
.10 \\
.07 \\
.05\end{array}$ & $\begin{array}{l}.00 \\
.00 \\
.00 \\
.00 \\
.00 \\
.00\end{array}$ & $\begin{array}{l}.00 \\
.01 \\
.01 \\
.02 \\
.04 \\
.08\end{array}$ & $\begin{array}{r}.04 \\
.10 \\
.50 \\
6.0\end{array}$ & $\begin{array}{l}.54 \\
.47 \\
.99 \\
.91 \\
.65 \\
.71\end{array}$ & $\begin{array}{l}.30 \\
.32 \\
.21 \\
.14 \\
.13\end{array}$ & $\begin{array}{l}.07 \\
.11 \\
.13 \\
.09 \\
.07 \\
.08\end{array}$ & $\begin{array}{l}.00 \\
.01 \\
.00 \\
.00 \\
.00\end{array}$ & $\begin{array}{l}.00 \\
.00 \\
.00 \\
.00 \\
.00 \\
.00\end{array}$ & $\begin{array}{l}.00 \\
.00 \\
.00 \\
.00 \\
.00 \\
.00\end{array}$ & $\begin{array}{l}.00 \\
.00 \\
.00 \\
.00 \\
.00\end{array}$ \\
\hline
\end{tabular}

Daily mean specific conductance, in microsiemens per centimeter at 25 degrees Celsius

\begin{tabular}{|c|c|c|c|c|c|c|c|c|c|c|c|c|}
\hline $\begin{array}{l}1 \\
2 \\
3 \\
4 \\
5\end{array}$ & $\begin{array}{l}--- \\
--- \\
--- \\
--- \\
---\end{array}$ & $\begin{array}{l}--- \\
--- \\
--- \\
---\end{array}$ & $\begin{array}{l}--- \\
--- \\
--- \\
---\end{array}$ & $\begin{array}{l}--- \\
--- \\
--- \\
--- \\
---\end{array}$ & $\begin{array}{l}--- \\
--- \\
-- \\
--- \\
--\end{array}$ & $\begin{array}{l}-- \\
-- \\
-- \\
-- \\
--\end{array}$ & $\begin{array}{l}--- \\
--- \\
--- \\
-- \\
---\end{array}$ & $\begin{array}{l}--- \\
-- \\
-- \\
--\end{array}$ & $\begin{array}{l}--- \\
726 \\
728 \\
722 \\
735\end{array}$ & $\begin{array}{l}\frac{\mathrm{a}}{\mathrm{a}} \text { ' } \\
\frac{\mathrm{a}}{\mathrm{a}} \text { ' } \\
\frac{\mathrm{a}}{\mathrm{a}} \text { ' }\end{array}$ & $\begin{array}{l}\frac{a}{a} / \\
\frac{a}{a} / \\
\frac{a}{a} /\end{array}$ & $\begin{array}{l}\frac{\mathrm{a}}{\mathrm{a}} / \\
\frac{\mathrm{a}}{\mathrm{a}} / \\
\frac{\mathrm{a}}{\mathrm{a}} /\end{array}$ \\
\hline $\begin{array}{r}6 \\
7 \\
8 \\
9 \\
10\end{array}$ & $\begin{array}{l}--- \\
--- \\
--- \\
--- \\
---\end{array}$ & $\begin{array}{l}--- \\
--- \\
--- \\
--- \\
---\end{array}$ & $\begin{array}{l}--2 \\
-- \\
--0 \\
--\end{array}$ & $\begin{array}{l}-- \\
=-- \\
--- \\
-- \\
--\end{array}$ & $\begin{array}{l}-- \\
-- \\
--- \\
-- \\
--\end{array}$ & $\begin{array}{l}-- \\
-- \\
--- \\
--- \\
--\end{array}$ & $\begin{array}{l}-- \\
-- \\
-- \\
-- \\
--\end{array}$ & $\begin{array}{l}--- \\
-- \\
-- \\
--\end{array}$ & $\begin{array}{l}734 \\
742 \\
744 \\
724 \\
737\end{array}$ & $\frac{a /}{\frac{a}{a} /}$ & $\frac{a}{a}$ d & $\begin{array}{l}\frac{\mathrm{a}}{\mathrm{a}} / \\
\frac{\mathrm{a}}{\mathrm{a}} / \\
\frac{\mathrm{a}}{\mathrm{a}} / \\
\mathrm{a} /\end{array}$ \\
\hline $\begin{array}{l}11 \\
12 \\
13 \\
14 \\
15\end{array}$ & $\begin{array}{l}--- \\
--- \\
--- \\
--- \\
---\end{array}$ & $\begin{array}{l}--- \\
--- \\
--- \\
--- \\
---\end{array}$ & $\begin{array}{l}--- \\
--- \\
--- \\
---\end{array}$ & $\begin{array}{l}--- \\
-- \\
--- \\
-- \\
--\end{array}$ & $\begin{array}{l}--- \\
-- \\
--1 \\
-- \\
--\end{array}$ & $\begin{array}{l}--- \\
-- \\
--- \\
-- \\
--\end{array}$ & $\begin{array}{l}-- \\
- \\
- \\
- \\
-\end{array}$ & $\begin{array}{l}--- \\
-17 \\
713 \\
708\end{array}$ & $\begin{array}{l}748 \\
757 \\
782 \\
805 \\
793\end{array}$ & $\begin{array}{l}\frac{\mathrm{a}}{\mathrm{a}} \text { ' } \\
\frac{\mathrm{a}}{\mathrm{a}} \mathrm{l} \\
\frac{\mathrm{a}}{\mathrm{a}} \mathrm{l}\end{array}$ & $\begin{array}{l}\frac{a}{a} \text { l } \\
\frac{a}{a} \text { l } \\
\frac{a}{a} /\end{array}$ & $\frac{a^{\prime} /}{\frac{a}{a} /}$ \\
\hline $\begin{array}{l}16 \\
17 \\
18 \\
19 \\
20\end{array}$ & $\begin{array}{l}--- \\
--- \\
--- \\
--- \\
---\end{array}$ & $\begin{array}{l}--- \\
--- \\
--- \\
-- \\
---\end{array}$ & $\begin{array}{l}=- \\
=- \\
-- \\
-- \\
--\end{array}$ & $\begin{array}{l}--- \\
--- \\
--- \\
--- \\
---\end{array}$ & $\begin{array}{l}=-- \\
=-- \\
--- \\
--- \\
--0\end{array}$ & $\begin{array}{l}--- \\
--0 \\
-- \\
--\end{array}$ & $\begin{array}{l}-- \\
-- \\
-- \\
-- \\
-\cdots\end{array}$ & $\begin{array}{l}701 \\
699 \\
697 \\
694 \\
693\end{array}$ & $\begin{array}{l}790 \\
791 \\
787 \\
775 \\
819\end{array}$ & $\begin{array}{l}\frac{\mathrm{a}}{\mathrm{a}} \text { ' } \\
\frac{\mathrm{a}}{\mathrm{a}} \mathrm{l} \\
\frac{\mathrm{a}}{\mathrm{a}} \mathrm{l}\end{array}$ & 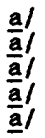 & $\begin{array}{l}\frac{\mathrm{a} /}{\mathrm{a}} / \\
\frac{\mathrm{a}}{\mathrm{a}} / \\
\frac{\mathrm{a}}{\mathrm{a}} /\end{array}$ \\
\hline $\begin{array}{l}21 \\
22 \\
23 \\
24 \\
25\end{array}$ & $\begin{array}{l}--- \\
--- \\
--- \\
--- \\
---\end{array}$ & $\begin{array}{l}--- \\
--- \\
--- \\
--- \\
---\end{array}$ & $\begin{array}{l}=- \\
-- \\
--\end{array}$ & $\begin{array}{l}--- \\
-- \\
--- \\
--- \\
---\end{array}$ & $\begin{array}{l}--- \\
--- \\
--- \\
--- \\
---\end{array}$ & $\begin{array}{l}-- \\
-- \\
--- \\
--- \\
---\end{array}$ & $\begin{array}{l}--- \\
--- \\
--- \\
-- \\
---\end{array}$ & $\begin{array}{l}692 \\
689 \\
692 \\
693 \\
690\end{array}$ & $\begin{array}{l}--- \\
-- \\
-- \\
-- \\
--\end{array}$ & $\begin{array}{l}\frac{\mathrm{a}}{\mathrm{a}} / \\
\frac{\mathrm{a}}{\mathrm{a}} / \\
\frac{\mathrm{a}}{\mathrm{a}} /\end{array}$ & 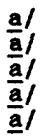 & $\begin{array}{l}\frac{\mathrm{a} /}{\mathrm{a}} / \\
\frac{\mathrm{a}}{\mathrm{a}} / \\
\frac{\mathrm{a}}{\mathrm{a}} /\end{array}$ \\
\hline $\begin{array}{l}26 \\
27 \\
28 \\
29 \\
30 \\
31\end{array}$ & $\begin{array}{l}-- \\
=-- \\
--- \\
--- \\
-- \\
--\end{array}$ & $\begin{array}{l}--- \\
--- \\
--- \\
--- \\
---\end{array}$ & $\begin{array}{l}--- \\
--- \\
--- \\
--- \\
---\end{array}$ & $\begin{array}{l}--- \\
-- \\
--- \\
-- \\
--\end{array}$ & $\begin{array}{l}--- \\
--- \\
--\infty\end{array}$ & 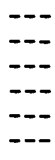 & $\begin{array}{l}-- \\
-- \\
-- \\
--\end{array}$ & $\begin{array}{l}691 \\
687 \\
689 \\
-- \\
-\end{array}$ & $\begin{array}{l}\text { al } \\
=- \\
\frac{a}{a} / \\
\frac{a}{a} /\end{array}$ & $\begin{array}{l}\frac{\mathrm{a}}{\mathrm{a}} \text { ' } \\
\frac{\mathrm{a}}{\mathrm{a}} \mathrm{l} \\
\frac{\mathrm{a}}{\mathrm{a}} \text { ' } \\
\frac{\mathrm{a}}{\mathrm{a}} \mathrm{l}\end{array}$ & 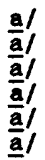 & $\begin{array}{l}\frac{\mathrm{a}}{\mathrm{a}} / \\
\frac{\mathrm{a}}{\mathrm{a}} / \\
\frac{\mathrm{a}}{\mathrm{a}} /\end{array}$ \\
\hline
\end{tabular}

a/ No discharge 

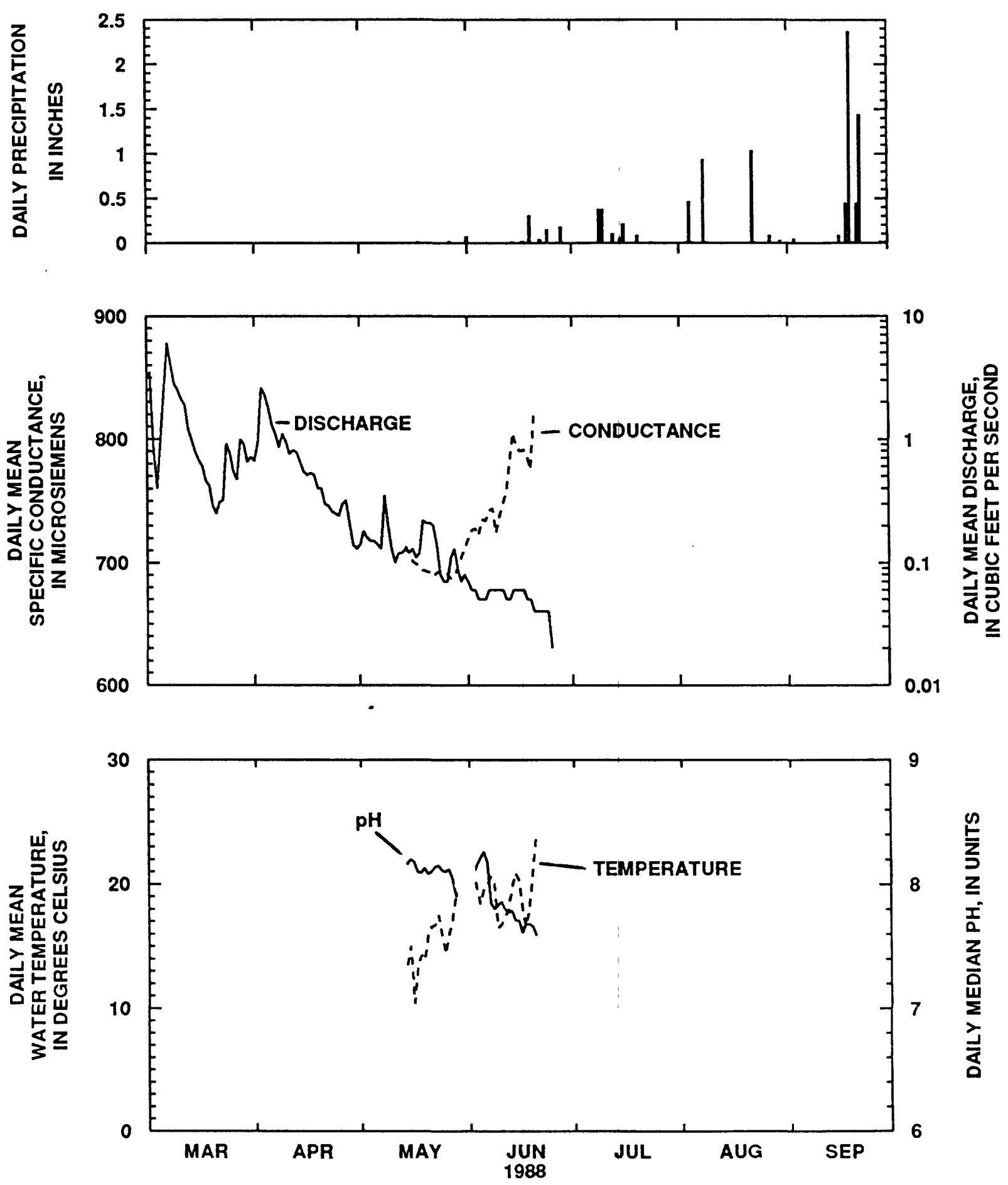

Figure 2.--Daily precipitation, daily mean specific conductance, discharge, water temperature, and daily median $\mathrm{pH}$ at site BOOGD, Unnamed Creek near Luana,

March-September, 1988. 
Table 7.--Daily mean water temperature and median pH at site BOOGD, Unnamed Creek near Luana Iowa

Water year October 1987 to September 1988

[--, data not available to calculate mean values ]

\begin{tabular}{|c|c|c|c|c|c|c|c|c|c|c|c|c|}
\hline Day & oct & Nov & Dec & Jan & Feb & Mar & Apr & May & June & July & Aug & Sep \\
\hline & & & & ily n & water & perat & \multicolumn{6}{|c|}{ in degrees Celcius } \\
\hline $\begin{array}{l}1 \\
2 \\
3 \\
4 \\
5\end{array}$ & $\begin{array}{l}--- \\
--- \\
--- \\
---\end{array}$ & $\begin{array}{l}--- \\
--- \\
--- \\
---\end{array}$ & $\begin{array}{l}--- \\
--- \\
--- \\
--- \\
---\end{array}$ & $\begin{array}{l}--- \\
--- \\
--- \\
--- \\
---\end{array}$ & $\begin{array}{l}--- \\
--- \\
--- \\
--- \\
---\end{array}$ & $\begin{array}{l}--- \\
--- \\
--- \\
--- \\
---\end{array}$ & $\begin{array}{l}--- \\
--- \\
--- \\
--- \\
---\end{array}$ & $\begin{array}{l}--- \\
--- \\
--- \\
--- \\
---\end{array}$ & $\begin{array}{r}--- \\
21.4 \\
19.7 \\
18.4 \\
19.6\end{array}$ & $\begin{array}{l}\frac{\mathrm{a} /}{\mathrm{a}} \text { ! } \\
\frac{\mathrm{a}}{\mathrm{a}} \text { ! } \\
\frac{\mathrm{a}}{} \text { l }\end{array}$ & $\begin{array}{l}\frac{a}{\mathrm{a}} \text { ' } \\
\frac{\mathrm{a}}{\mathrm{a}} \text { ! } \\
\frac{\mathrm{a}}{\mathrm{a}} \text { ' }\end{array}$ & $\begin{array}{l}\frac{\mathrm{a} /}{\mathrm{a}} \text { ' } \\
\frac{\mathrm{a}}{\mathrm{a}} \text { ' } \\
\frac{\mathrm{a}}{\mathrm{a}} /\end{array}$ \\
\hline $\begin{array}{r}6 \\
7 \\
8 \\
9 \\
10\end{array}$ & $\begin{array}{l}--- \\
--- \\
--- \\
---\end{array}$ & $\begin{array}{l}--- \\
--- \\
--- \\
---\end{array}$ & $\begin{array}{l}--- \\
--- \\
--- \\
--- \\
---\end{array}$ & $\begin{array}{l}--- \\
--- \\
--- \\
--- \\
---\end{array}$ & $\begin{array}{l}--- \\
--- \\
--- \\
--- \\
---\end{array}$ & $\begin{array}{l}--- \\
--- \\
--- \\
--- \\
---\end{array}$ & $\begin{array}{l}--- \\
--- \\
--- \\
--- \\
---\end{array}$ & $\begin{array}{l}--- \\
--- \\
--- \\
--- \\
---\end{array}$ & $\begin{array}{l}20.0 \\
20.6 \\
20.1 \\
16.5 \\
16.7\end{array}$ & $\begin{array}{l}\frac{\mathrm{a}}{\mathrm{a}} \text { ! } \\
\frac{\mathrm{a}}{\mathrm{a}} \text { ! } \\
\frac{\mathrm{a}}{\mathrm{a}} \text { ! }\end{array}$ & $\begin{array}{l}\frac{a}{a} / \\
\frac{a}{a} / \\
\frac{a}{a} \text { l } \\
\underline{a} /\end{array}$ & $\begin{array}{l}\frac{\mathrm{a} /}{\mathrm{a}} / \\
\overline{\mathrm{a}} / \\
\frac{\mathrm{a}}{\mathrm{a}} /\end{array}$ \\
\hline $\begin{array}{l}11 \\
12 \\
13 \\
14 \\
15\end{array}$ & $\begin{array}{l}--- \\
--- \\
--- \\
--- \\
---\end{array}$ & $\begin{array}{l}--- \\
--- \\
--- \\
---\end{array}$ & $\begin{array}{l}--- \\
--- \\
--- \\
--- \\
---\end{array}$ & $\begin{array}{l}--- \\
--- \\
--- \\
--- \\
---\end{array}$ & $\begin{array}{l}--- \\
--- \\
--- \\
--- \\
---\end{array}$ & $\begin{array}{l}--- \\
--- \\
--- \\
--- \\
--\end{array}$ & $\begin{array}{l}--- \\
--- \\
--- \\
--- \\
---\end{array}$ & $\begin{array}{r}--- \\
--- \\
--- \\
13.5 \\
15.0\end{array}$ & $\begin{array}{l}17.4 \\
17.9 \\
19.6 \\
20.9 \\
20.4\end{array}$ & $\begin{array}{l}\frac{\mathrm{a}}{\mathrm{a}} \text { ' } \\
\frac{\mathrm{a}}{\mathrm{a}} \text { ! } \\
\frac{\mathrm{a}}{\mathrm{a}} \text { ! }\end{array}$ & $\begin{array}{l}\frac{\mathrm{a}}{\mathrm{a}} \text { l } \\
\frac{\mathrm{a}}{\mathrm{a}} \text { ! } \\
\frac{\mathrm{a}}{\mathrm{a}} \text { l }\end{array}$ & $\begin{array}{l}\frac{a}{a} \prime \\
\frac{a}{a} / \\
\frac{a}{a} /\end{array}$ \\
\hline $\begin{array}{l}16 \\
17 \\
18 \\
19 \\
20\end{array}$ & $\begin{array}{l}--- \\
--- \\
--- \\
---\end{array}$ & $\begin{array}{l}--- \\
--- \\
--- \\
---\end{array}$ & $\begin{array}{l}--- \\
--- \\
--- \\
--- \\
---\end{array}$ & $\begin{array}{l}--- \\
--- \\
--- \\
--- \\
---\end{array}$ & $\begin{array}{l}--- \\
--- \\
--- \\
--- \\
---\end{array}$ & $\begin{array}{l}--- \\
--- \\
--- \\
--- \\
---\end{array}$ & $\begin{array}{l}--- \\
--- \\
--- \\
--- \\
---\end{array}$ & $\begin{array}{l}10.4 \\
13.4 \\
14.3 \\
13.9 \\
16.0\end{array}$ & $\begin{array}{l}18.3 \\
16.6 \\
17.9 \\
20.8 \\
23.8\end{array}$ & $\begin{array}{l}\frac{\mathrm{a} /}{\mathrm{a}} \text { l } \\
\overline{\mathrm{a}} / \\
\frac{\mathrm{a}}{\mathrm{a}} \text { l }\end{array}$ & $\begin{array}{l}\frac{a}{\mathrm{a}} \text { l } \\
\frac{\mathrm{a}}{\mathrm{a}} \text { ! } \\
\underline{\mathrm{a}} \text { ! }\end{array}$ & $\begin{array}{l}\frac{\mathrm{a}}{\mathbf{a}} / \\
\frac{\mathrm{a}}{\mathrm{a}} / \\
\frac{\mathrm{a}}{\mathrm{a}} /\end{array}$ \\
\hline $\begin{array}{l}21 \\
22 \\
23 \\
24 \\
25\end{array}$ & $\begin{array}{l}--- \\
--- \\
--- \\
---\end{array}$ & $\begin{array}{l}--- \\
-- \\
-- \\
--\end{array}$ & $\begin{array}{l}--- \\
--- \\
--- \\
--- \\
---\end{array}$ & $\begin{array}{l}--- \\
--- \\
--- \\
--- \\
---\end{array}$ & $\begin{array}{l}--- \\
--- \\
--- \\
--- \\
---\end{array}$ & $\begin{array}{l}--- \\
--- \\
--- \\
--- \\
---\end{array}$ & $\begin{array}{l}--- \\
--- \\
--- \\
--- \\
---\end{array}$ & $\begin{array}{l}16.6 \\
16.7 \\
17.5 \\
15.8 \\
14.4\end{array}$ & $\begin{array}{l}--- \\
--- \\
--- \\
--- \\
---\end{array}$ & $\begin{array}{l}\frac{\mathrm{a}}{\mathrm{a}} \text { ' } \\
\frac{\mathrm{a}}{\mathrm{a}} \text { ' } \\
\frac{\mathrm{a}}{\mathrm{a}} \text { ' }\end{array}$ & $\begin{array}{l}\frac{a}{\mathrm{a}} \text { ' } \\
\frac{\mathrm{a}}{\mathrm{a}} \text { ! } \\
\frac{\mathrm{a}}{\mathrm{a}} \text { ' }\end{array}$ & $\begin{array}{l}\frac{a}{a} / \\
\frac{a}{a} / \\
\frac{a}{a} /\end{array}$ \\
\hline $\begin{array}{l}26 \\
27 \\
28 \\
29 \\
30 \\
31\end{array}$ & $\begin{array}{l}--- \\
-- \\
-- \\
-- \\
---\end{array}$ & $\begin{array}{l}--- \\
--- \\
--- \\
---\end{array}$ & $\begin{array}{l}--- \\
--- \\
--- \\
--- \\
--- \\
---\end{array}$ & $\begin{array}{l}--- \\
--- \\
--- \\
--- \\
--- \\
---\end{array}$ & $\begin{array}{l}--- \\
--- \\
--- \\
---\end{array}$ & $\begin{array}{l}--- \\
--- \\
--- \\
--- \\
---\end{array}$ & $\begin{array}{l}--- \\
--- \\
--- \\
---\end{array}$ & $\begin{array}{r}16.1 \\
17.2 \\
19.2 \\
--- \\
--- \\
---\end{array}$ & $\begin{array}{l}\text { al } \\
=- \\
\frac{a}{a} / \\
\frac{a}{a} /\end{array}$ & $\begin{array}{l}\frac{a}{\mathrm{a}} \text { l } \\
\frac{\mathrm{a}}{\mathrm{a}} \text { ! } \\
\frac{\mathrm{a}}{\mathrm{a}} \text { ! } \\
\frac{\mathrm{a}}{\mathrm{a}} \text { l }\end{array}$ & $\begin{array}{l}\frac{a}{a} \text { l } \\
\frac{a}{a} \text { l } \\
\frac{a}{a} \text { l } \\
\frac{a}{a} \text { l }\end{array}$ & $\begin{array}{l}\frac{a}{a} / \\
\frac{a}{a} / \\
\frac{a}{a} /\end{array}$ \\
\hline
\end{tabular}

Daily median $\mathrm{pH}$, in units

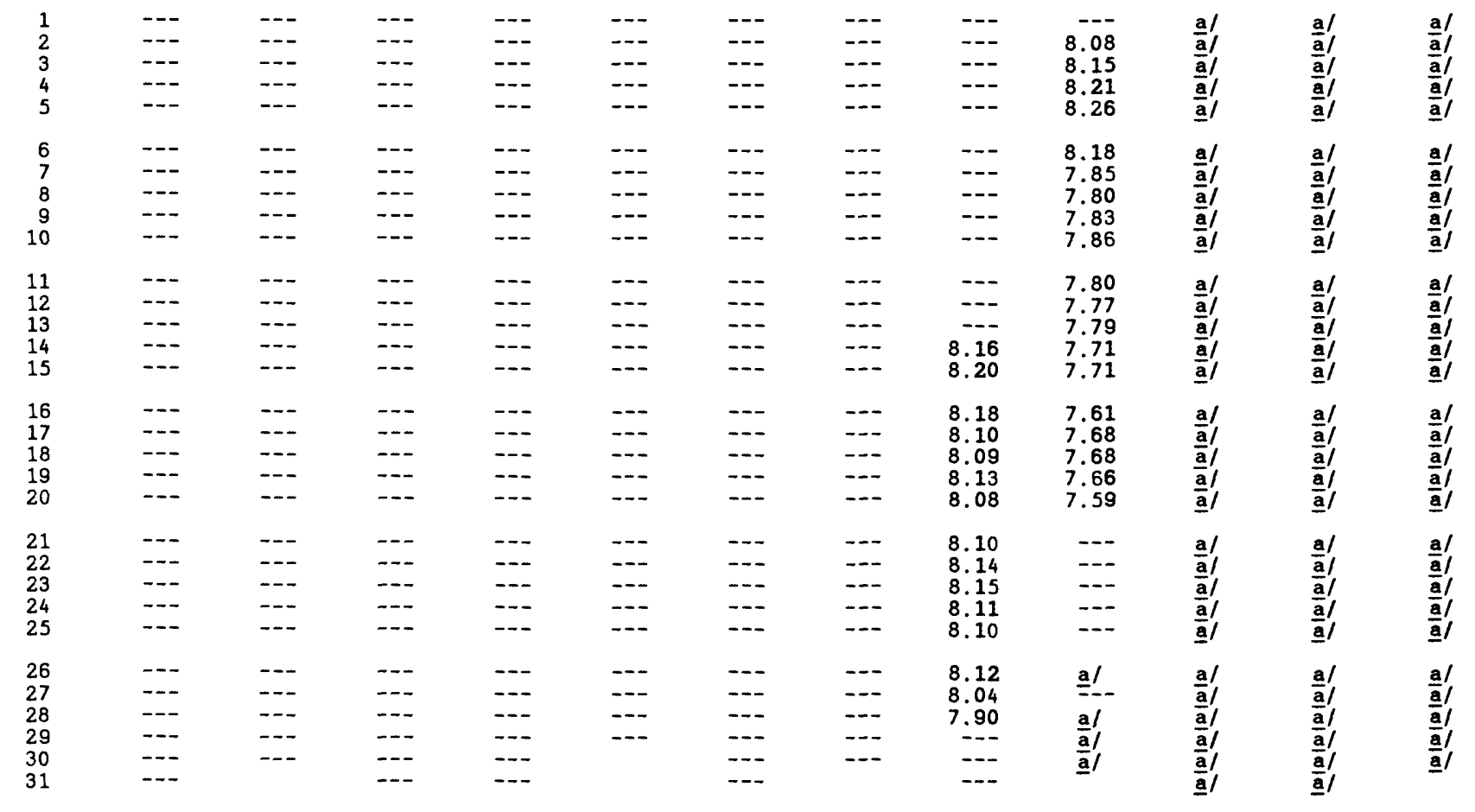


Table 8.-Field measurements at monthly monitoring sites in the Big Spring Basin

$\left[\mathrm{ft}^{3} / \mathrm{s}\right.$, cubic feet per second; $\mu \mathrm{S} / \mathrm{cm}$, microsiemens per centimeter; ${ }^{\circ} \mathrm{C}$,degrees celsius; mg/L, milligrams per liter; --, missing data; $>$, greater than]

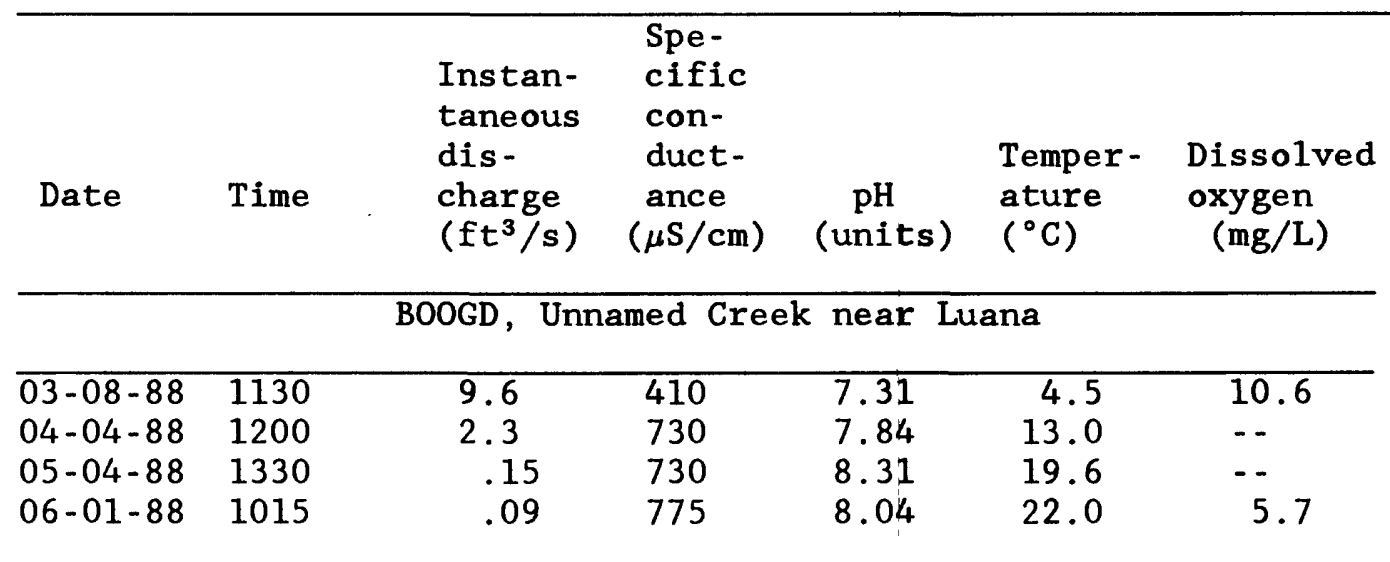

RC2, Roberts Creek above St. Olaf

\begin{tabular}{rrrrrrr}
\hline $03-08-88$ & 1330 & 173 & 480 & 7.72 & 2.0 & 12.0 \\
$04-05-88$ & 0900 & 80 & 650 & 8.30 & 13.0 & 9.8 \\
$05-04-88$ & 1455 & 15 & 650 & 8.85 & 20.5 & -- \\
$06-01-88$ & 1510 & 5.7 & 700 & 8.60 & 28.0 & 14.2 \\
$07-01-88$ & 1100 & 2.9 & 580 & 8.90 & 27.0 & 10.6 \\
& & & & & & \\
$08-05-88$ & 0810 & .97 & 590 & 7.74 & 23.0 & 7.5 \\
$09-06-88$ & 1000 & .08 & 540 & 8.20 & 12.5 & 18.2 \\
$09-12-88$ & 1350 & .04 & 365 & 9.40 & 26.0 & $>20.0$ \\
$09-20-88$ & 0950 & 22 & 450 & 8.10 & 16.0 & $\ldots$ \\
$09-22-88$ & 1340 & 78 & 504 & 7.64 & 17.5 & $\cdots$ \\
\hline
\end{tabular}

Big Spring

\begin{tabular}{lllllll}
\hline $03-08-88$ & 0930 & -- & 550 & 7.20 & 8.0 & 9.3 \\
$04-04-88$ & 1330 & -- & 730 & 7.24 & 9.0 & 9.2 \\
$05-04-88$ & 1055 & -- & 740 & 7.23 & 10.0 & -- \\
$06-01-88$ & 1230 & -- & 780 & 7.24 & 10.0 & 8.9 \\
$07-01-88$ & 1330 & -- & 750 & 7.10 & 10.0 & 9.6 \\
& & & & & & \\
$08-04-88$ & 1545 & -- & 750 & 7.22 & 11.0 & 10.2 \\
$09-06-88$ & 1200 & -- & 690 & 7.21 & 10.5 & 9.6 \\
$09-20-88$ & 1230 & -- & -- & -- & -- & -- \\
\hline
\end{tabular}


Table 9.--Concentrations of major ions at the monthly monitoring sites in the Big Spring Basin [Dissolved constituents are in milligrams per liter; - - data not collected]

\begin{tabular}{|c|c|c|c|c|c|c|c|c|c|c|}
\hline Date & Time & Calcium & $\begin{array}{l}\text { Mag- } \\
\text { ne- } \\
\text { sium }\end{array}$ & $\begin{array}{l}\text { So- } \\
\text { dium }\end{array}$ & $\begin{array}{l}\text { Pot- } \\
\text { tas- } \\
\text { sium }\end{array}$ & $\begin{array}{l}\text { Bi- } \\
\text { carbo- } \\
\text { nate }\end{array}$ & $\begin{array}{l}\text { Car- } \\
\text { bo- } \\
\text { nate }\end{array}$ & $\begin{array}{l}\text { Sul- } \\
\text { fate }\end{array}$ & $\begin{array}{l}\text { Chlo- } \\
\text { ride }\end{array}$ & Silica \\
\hline & \multicolumn{10}{|c|}{ BOOGD, Unnamed Creek near Luana } \\
\hline $\begin{array}{l}03-08-88 \\
04-04-88 \\
06-01-88\end{array}$ & $\begin{array}{l}1130 \\
1200 \\
1015\end{array}$ & $\begin{array}{l}44 \\
78 \\
79\end{array}$ & $\begin{array}{l}19 \\
29 \\
33\end{array}$ & $\begin{array}{l}5.4 \\
9.8 \\
8.4\end{array}$ & $\begin{array}{l}5.1 \\
4.4 \\
2.2\end{array}$ & $\begin{array}{l}182 \\
305 \\
366\end{array}$ & $\begin{array}{l}0 \\
0 \\
0\end{array}$ & $\begin{array}{l}22 \\
31 \\
32\end{array}$ & $\begin{array}{l}12 \\
26 \\
24\end{array}$ & $\begin{array}{l}10 \\
13 \\
18\end{array}$ \\
\hline \multicolumn{11}{|c|}{ RC2, Roberts Creek above St. Olaf } \\
\hline $\begin{array}{l}03-08-88 \\
04-05-88 \\
06-23-88 \\
06-24-88 \\
07-01-88\end{array}$ & $\begin{array}{l}1330 \\
0900 \\
1800 \\
0600 \\
1100\end{array}$ & $\begin{array}{l}53 \\
76 \\
52 \\
54 \\
51\end{array}$ & $\begin{array}{l}22 \\
33 \\
34 \\
33 \\
36\end{array}$ & $\begin{array}{c}7.5 \\
7.2 \\
10 \\
9.5 \\
10\end{array}$ & $\begin{array}{l}5.1 \\
3.6 \\
3.8 \\
3.5 \\
4.0\end{array}$ & $\begin{array}{l}220 \\
237 \\
-- \\
-- \\
251\end{array}$ & $\begin{array}{r}0 \\
36 \\
-- \\
\cdots \\
30\end{array}$ & $\begin{array}{l}26 \\
26 \\
37 \\
33 \\
38\end{array}$ & $\begin{array}{l}16 \\
22 \\
23 \\
22 \\
26\end{array}$ & $\begin{array}{r}11 \\
15 \\
9.0 \\
9.0 \\
7.5\end{array}$ \\
\hline $\begin{array}{l}08-05-88 \\
09-06-88 \\
09-20-88\end{array}$ & $\begin{array}{l}0810 \\
1000 \\
0950\end{array}$ & $\begin{array}{l}57 \\
85 \\
--\end{array}$ & $\begin{array}{l}29 \\
30 \\
--\end{array}$ & $\begin{array}{c}10 \\
8.2 \\
--\end{array}$ & $\begin{array}{l}5.0 \\
4.8 \\
--\end{array}$ & $\begin{array}{l}305 \\
354 \\
--\end{array}$ & $\begin{array}{l}0 \\
0 \\
\cdots\end{array}$ & $\begin{array}{l}30 \\
24 \\
40\end{array}$ & $\begin{array}{l}24 \\
23 \\
20\end{array}$ & $\begin{array}{l}8.3 \\
-- \\
--\end{array}$ \\
\hline \multicolumn{11}{|c|}{ Big Spring } \\
\hline $\begin{array}{l}03-08-88 \\
04-04-88 \\
07-01-88 \\
09-06-88 \\
09-20-88\end{array}$ & $\begin{array}{l}0930 \\
1330 \\
1330 \\
1200 \\
1230\end{array}$ & $\begin{array}{l}64 \\
84 \\
83 \\
96 \\
--\end{array}$ & $\begin{array}{l}25 \\
34 \\
37 \\
39 \\
--\end{array}$ & $\begin{array}{l}6.5 \\
7.1 \\
13 \\
12 \\
--\end{array}$ & $\begin{array}{l}4.7 \\
3.0 \\
2.6 \\
3.9 \\
--\end{array}$ & $\begin{array}{l}264 \\
384 \\
384 \\
390 \\
--\end{array}$ & $\begin{array}{l}0 \\
0 \\
0 \\
0 \\
-\end{array}$ & $\begin{array}{l}23 \\
24 \\
35 \\
32 \\
37\end{array}$ & $\begin{array}{l}14 \\
19 \\
24 \\
24 \\
23\end{array}$ & $\begin{array}{c}13 \\
17 \\
5.8 \\
\cdots \\
--\end{array}$ \\
\hline
\end{tabular}


Table 10.--Selected nitrogen, phosphorus and carbon species at month1y monitoring sites in the Big Spring Basin

[Dissolved constituents in milligrams per liter; $\mathrm{N}$, nitrogen; $\mathrm{P}$, phosphorus; $C$, carbon; --, data not collected; <, less than]

\begin{tabular}{|c|c|c|c|c|c|c|}
\hline Date & Time & $\begin{array}{l}\text { Nitrate } \\
\text { plus } \\
\text { nitrite, } \\
\text { (as N) }\end{array}$ & $\begin{array}{l}\text { Ammonia, } \\
\text { (as N) }\end{array}$ & $\begin{array}{l}\text { Organic } \\
\text { nitrogen, } \\
\text { (as N) }\end{array}$ & $\begin{array}{l}\text { Ortho- } \\
\text { phos- } \\
\text { phorus, } \\
\text { (as P) }\end{array}$ & $\begin{array}{l}\text { Total } \\
\text { Organic } \\
\text { carbon } \\
(\text { as } \mathrm{C})\end{array}$ \\
\hline
\end{tabular}

BO0GD Unnamed Creek near Luana

\begin{tabular}{rrrrrrl}
\hline $03-08-88$ & 1130 & 7.6 & 0.2 & 0.8 & -- & 3.4 \\
$04-05-88$ & 1200 & 11 & .1 & .7 & -- & 2.8 \\
$05-04-88$ & 1330 & 14 & $<.1$ & .1 & $<0.1$ & -- \\
$06-01-88$ & 1015 & 13 & .5 & .4 & .1 & --
\end{tabular}

RC2 Roberts Creek above St. Olaf

\begin{tabular}{|c|c|c|c|c|c|c|}
\hline $03-08-88$ & 1330 & 7.2 & 0.4 & 1.1 & -- & 3.1 \\
\hline $04-05-88$ & 0900 & 15 & $<.1$ & .3 & -- & 1.7 \\
\hline $05-04-88$ & 1455 & 6.1 & $<.1$ & .4 & 0.2 & - - \\
\hline $06-01-88$ & 1510 & 3.3 & $<.1$ & $<.1$ & - & - \\
\hline $07-01-88$ & 1100 & .2 & .2 & .5 & -- & 4.2 \\
\hline $08-05-88$ & 0810 & 1.5 & $<.1$ & .4 & - - & 4.1 \\
\hline $09-06-88$ & 1000 & 2.2 & $<.1$ & $<.1$ & $<.1$ & -- \\
\hline $09-20-88$ & 0950 & .5 & .2 & .7 & .2 & -- \\
\hline
\end{tabular}

Big Spring

\begin{tabular}{rrrrrrl}
\hline $03-08-88$ & 0930 & 8.6 & 0.1 & 0.6 & -- & 2.6 \\
$04-04-88$ & 1330 & 11 & .3 & .3 &.- & 1.2 \\
$05-04-88$ & 1055 & 10 & $<.1$ & $<.1$ & $<0.1$ &.- \\
$06-01-88$ & 1230 & 9.7 & $<.1$ & .2 & -- &.- \\
$07-01-88$ & 1330 & 9.0 & $<.1$ & .2 & -- & 1.2 \\
& & & & & & \\
$08-04-88$ & 1545 & 8.5 & $<.1$ & $<.1$ &.- & 1.1 \\
$09-06-88$ & 1200 & 8.1 & $<.1$ & $<.1$ & .2 &.- \\
$09-20-88$ & 1230 & 7.5 & $<.1$ & .2 & .2 &.- \\
\hline
\end{tabular}


Two pesticides were detected in samples from Unnamed Creek near Luana (table 11). Atrazine was detected in four samples and alachlor was detected in one sample. Total recoverable atrazine concentrations ranged from 0.16 to $0.34 \mu \mathrm{g} / \mathrm{L}$. The greatest concentration occurred on March 8 . The total recoverable alachlor concentration was $0.11 \mu \mathrm{g} / \mathrm{L}$ in a sample collected on June 1 .

Sediment concentrations ranged from $22 \mathrm{mg} / \mathrm{L}$ on March 23 to $1,160 \mathrm{mg} / \mathrm{L}$ on May 8. During rainfall on May 8, sampled suspended-sediment concentrations increased from $76 \mathrm{mg} / \mathrm{L}$ at 0705 hours to $1,160 \mathrm{mg} / \mathrm{L}$ at 1740 hours (table 12).

\section{Discharge}

Roberts Creek

Daily mean discharge for site RC2, Roberts Creek above Saint 0laf, is listed in table 13 and illustrated graphically from March 1 to September 30 in figure 3. Maximum daily discharge $\left(190 \mathrm{ft}^{3} / \mathrm{s}\right)$ occurred on March 2 and generally decreased through spring and summer reaching a minimum of $0.02 \mathrm{ft}^{3} / \mathrm{s}$ on September 17 and 18. The median discharge at site RC2 for the 1988 water year was $7.7 \mathrm{ft}^{3} / \mathrm{s}$.

\section{Water quality}

Maximum specific conductance $(691 \mu \mathrm{S} / \mathrm{cm})$ was recorded the first day of monitor operation (June 1) at site RC2 and the minimum mean daily specific conductance $(393 \mu \mathrm{S} / \mathrm{cm}$ ) was on September 13 (table 13). The daily median $\mathrm{pH}$ at site RC2 varied from 7.23 to 8.82 units and daily mean water temperature varied from 15.3 to 30.2 degrees Celsius (table 14, fig. 3).

Analyses of water quality samples from site RC2 showed that calcium and magnesium were the predominant cations and bicarbonate was the predominant anion in solution (table 9). Sodium and potassium concentrations were $10 \mathrm{mg} / \mathrm{L}$ or less. Sulfate concentrations ranged from 24 to $40 \mathrm{mg} / \mathrm{L}$ and chloride concentrations ranged from 16 to $26 \mathrm{mg} / \mathrm{L}$. Silica concentrations were $15 \mathrm{mg} / \mathrm{L}$ or less. Nitrate plus nitrite was the major nitrogen species present at site RC2 (table 10) and ranged from 0.2 to $15 \mathrm{mg} / \mathrm{L}$ as nitrogen. Ammonia nitrogen was detected in three of eight samples. The maximum concentration was 0.4 $\mathrm{mg} / \mathrm{L}$. Organic nitrogen was detected in six of eight samples and had a maximum concentration of $1.1 \mathrm{mg} / \mathrm{L}$ as nitrogen.

Atrazine was found in concentrations greater than the detection limit in seven of eight samples (table 11 ). Total recoverable atrazine concentrations ranged from 0.11 to $0.72 \mu \mathrm{g} / \mathrm{L}$. Total recoverable cyanazine concentrations exceeded the detection limits in June to September. The maximum concentration $(0.72 \mu \mathrm{g} / \mathrm{L})$ was in July. Total recoverable metolachlor and alachlor concentrations both exceeded the detection limit in one sample. Total recoverable metribuzin, butylate, and trifluralin were not detected.

Suspended sediment concentrations at site RC2 ranged from less than 10.0 $\mathrm{mg} / \mathrm{L}$ on April 23 and 24 to $810 \mathrm{mg} / \mathrm{L}$ on September 22 during a rainfall event (table 15). From April 1 to August 31, suspended sediment concentration generally were less than $100 \mathrm{mg} / \mathrm{L}$ (fig. 4). Occasionally concentrations exceeded $100 \mathrm{mg} / \mathrm{L}$ during this period of declining stream discharge. During September, suspended sediment concentrations generally exceeded $100 \mathrm{mg} / \mathrm{L}$. 
Table 11.--Selected pesticides at monthly monitoring sites in the Big Spring Basin

[Total recoverable constituents in micrograms per liter; <, less than]

\begin{tabular}{lrrrrrrrr}
\hline Date & Time & $\begin{array}{c}\text { Atra- } \\
\text { zine }\end{array}$ & $\begin{array}{c}\text { Cyana- } \\
\text { zine }\end{array}$ & $\begin{array}{c}\text { Metola- Ala- } \\
\text { chlor } \\
\text { chlor }\end{array}$ & $\begin{array}{c}\text { Metri- } \\
\text { buzin }\end{array}$ & $\begin{array}{c}\text { Buty- } \\
\text { late } \\
\text { flur- } \\
\text { alin }\end{array}$ \\
\hline \multicolumn{7}{c}{ BOOGD, Unnamed Creek near Luana } \\
\hline $03-08-88$ & 1130 & 0.34 & $<0.1$ & $<0.1$ & $<0.1$ & $<0.1$ & $<0.1$ & $<0.1$ \\
$04-05-88$ & 1200 & .27 & $<.1$ & $<.1$ & $<.1$ & $<.1$ & $<.1$ & $<.1$ \\
$05-04-88$ & 1015 & .16 & $<.1$ & $<.1$ & $<.1$ & $<.1$ & $<.1$ & $<.1$ \\
$06-01-88$ & 1015 & .22 & $<.1$ & $<.1$ & .11 & $<.1$ & $<.1$ & $<.1$
\end{tabular}

RC2, Roberts Creek above St. Olaf

\begin{tabular}{|c|c|c|c|c|c|c|c|c|}
\hline $03-08-88$ & 1330 & 0.24 & $<0.1$ & $<0.1$ & $<0.1$ & $<0.1$ & $<0.1$ & $<0.1$ \\
\hline $04-05-88$ & 0900 & .11 & $<.1$ & $<.1$ & $<.1$ & $<.1$ & $<.1$ & $<.1$ \\
\hline $05-04-88$ & 1455 & .40 & $<.1$ & $<.1$ & $<.1$ & $<.1$ & & \\
\hline $06-01-88$ & 1510 & .72 & .23 & $<.1$ & $<.1$ & $<.1$ & $<.1$ & \\
\hline $07-01-88$ & 1100 & .41 & .72 & $<.1$ & $<.1$ & $<.1$ & $<.1$ & $<.1$ \\
\hline $08-05-88$ & 0810 & .42 & .51 & $<.1$ & .55 & $<.1$ & & \\
\hline $09-06-88$ & 1000 & .19 & $<.1$ & $<.1$ & $<.1$ & $<.1$ & $<.1$ & $<.1$ \\
\hline $09-20-88$ & 0950 & $<.1$ & $<.1$ & $<.1$ & $<.1$ & $<.1$ & $<.1$ & $<.1$ \\
\hline $09-22-88$ & 1340 & .51 & .12 & .11 & $<.1$ & $<.1$ & $<.1$ & $<.1$ \\
\hline & \multicolumn{8}{|c|}{ Big Spring } \\
\hline $03-08-88$ & 0930 & 0.19 & $<0.1$ & $<0.1$ & $<0.1$ & $<0.1$ & $<0.1$ & $<0.1$ \\
\hline $04-05-88$ & 1330 & .11 & $<.1$ & $<.1$ & $<.1$ & $<.1$ & $<.1$ & $<.1$ \\
\hline $05-04-88$ & 1055 & .26 & $<.1$ & $<.1$ & $<.1$ & $<.1$ & & $<.1$ \\
\hline $06-01-88$ & 1230 & .24 & $<.1$ & $<.1$ & $<.1$ & $<.1$ & & $<.1$ \\
\hline $07-01-88$ & 1330 & .12 & $<.1$ & $<.1$ & $<.1$ & $<.1$ & $<.1$ & $<.1$ \\
\hline $08-04-88$ & 1545 & $<.1$ & $<.1$ & $<.1$ & & $<.1$ & & $<.1$ \\
\hline $09-06-88$ & 1200 & .12 & $<.1$ & $<.1$ & $<.1$ & $<.1$ & $<.1$ & $<.1$ \\
\hline
\end{tabular}


Table 12. - Instantaneous discharge and suspended sediment concentrations

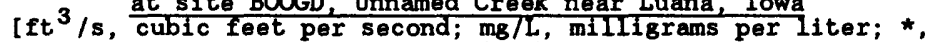

\begin{tabular}{|c|c|c|c|c|c|c|c|}
\hline Date & Time & $\begin{array}{l}\text { Instan- } \\
\text { taneous } \\
\text { discharge } \\
\text { (ft } 3 / \mathrm{s})\end{array}$ & $\begin{array}{l}\text { Suspended } \\
\text { sediment } \\
\text { (mg/L) }\end{array}$ & Date & Time & $\begin{array}{l}\text { Instan- } \\
\text { taneous } \\
\text { discharge } \\
\left(\mathrm{ft}^{3} / \mathrm{s}\right)\end{array}$ & $\begin{array}{l}\text { Suspended } \\
\text { sediment } \\
\text { (mg/L) }\end{array}$ \\
\hline $\begin{array}{l}3-01-88 \\
3-08-88 \\
3-23-88 \\
3-29-88 \\
3-31-88\end{array}$ & $\begin{array}{l}1135 \\
1130 \\
1200 \\
1100 \\
1230\end{array}$ & $\begin{array}{c}3.6 \\
8.8 \\
.27 \\
.91 \\
.64\end{array}$ & $\begin{array}{r}30 \\
129 \\
22 \\
38 \\
43\end{array}$ & $\begin{array}{l}5-08-88 \\
5-10-88 \\
5-13-88 \\
5-14-88 \\
5-17-88\end{array}$ & $\begin{array}{l}1740 \\
0903 \\
0915 \\
0735 \\
0925\end{array}$ & $\begin{array}{l}1.8 \\
.12 \\
.13 \\
.18 \\
.10\end{array}$ & $\begin{array}{r}1,160 \\
74 \\
69 \\
62 \\
59\end{array}$ \\
\hline $\begin{array}{l}4-04-88 \\
4-06-88 \\
4-13-88 \\
4-14-88 \\
4-16-88\end{array}$ & $\begin{array}{l}1145 \\
1230 \\
1345 \\
0950 \\
0930\end{array}$ & $\begin{array}{r}* 2.3 \\
\star 1.3 \\
.84 \\
.64 \\
.42\end{array}$ & $\begin{array}{l}50 \\
37 \\
58 \\
32 \\
32\end{array}$ & $\begin{array}{l}5-19-88 \\
5-21-88 \\
5-23-88 \\
5-25-88 \\
5-27-88\end{array}$ & $\begin{array}{l}0720 \\
0755 \\
0725 \\
0710 \\
0740\end{array}$ & $\begin{array}{l}.24 \\
.21 \\
.17 \\
.07 \\
.11\end{array}$ & $\begin{array}{r}78 \\
104 \\
78 \\
69 \\
199\end{array}$ \\
\hline $\begin{array}{l}4-18-88 \\
4-20-88 \\
4-22-88 \\
4-25-88 \\
4-26-88\end{array}$ & $\begin{array}{l}0840 \\
0845 \\
0825 \\
0905 \\
1650\end{array}$ & $\begin{array}{l}.58 \\
.53 \\
.27 \\
.24 \\
.46\end{array}$ & $\begin{array}{r}43 \\
89 \\
27 \\
28 \\
302\end{array}$ & $\begin{array}{l}5-31-88 \\
6-02-88 \\
6-04-88 \\
6-06-88 \\
6-08-88\end{array}$ & $\begin{array}{l}0825 \\
0712 \\
0705 \\
0715 \\
0730\end{array}$ & $\begin{array}{l}.10 \\
.06 \\
.05 \\
.05 \\
.06\end{array}$ & $\begin{array}{l}116 \\
139 \\
278 \\
133 \\
114\end{array}$ \\
\hline $\begin{array}{l}4-27-88 \\
4-29-88 \\
5-03-88 \\
5-05-88 \\
5-07-88\end{array}$ & $\begin{array}{l}0800 \\
0919 \\
0843 \\
0835 \\
0817\end{array}$ & $\begin{array}{l}.29 \\
.13 \\
.15 \\
.13 \\
.11\end{array}$ & $\begin{array}{l}37 \\
32 \\
54 \\
33 \\
60\end{array}$ & $\begin{array}{l}6-10-88 \\
6-14-88 \\
6-16-88 \\
6-18-88 \\
6-20-88\end{array}$ & $\begin{array}{l}0725 \\
0700 \\
0720 \\
0835 \\
0850\end{array}$ & $\begin{array}{l}.06 \\
.07 \\
.06 \\
.05 \\
.05\end{array}$ & $\begin{array}{r}278 \\
244 \\
228 \\
47 \\
95\end{array}$ \\
\hline $\begin{array}{l}5-08-88 \\
5-08-88\end{array}$ & $\begin{array}{l}0705 \\
1614\end{array}$ & $\begin{array}{l}.10 \\
.72\end{array}$ & $\begin{array}{r}76 \\
424\end{array}$ & $\begin{array}{l}6-22-88 \\
6-25-88\end{array}$ & $\begin{array}{l}0835 \\
0825\end{array}$ & $\begin{array}{l}.05 \\
.03\end{array}$ & $\begin{array}{r}118 \\
90\end{array}$ \\
\hline
\end{tabular}



Table 13. - - $\frac{\text { Daily mean discharge and specific conductance at site RC2, }}{\text { Roberts Creek above Saint Olaf Iowa }}$

[--- . data not available to calculate mean values]

\begin{tabular}{|c|c|c|c|c|c|c|c|c|c|c|c|c|}
\hline Day & Oct & Nov & Dec & Jan & Feb & Mar & Apr & May & June & July & Aug & Sept \\
\hline & \multicolumn{12}{|c|}{ Daily mean discharge, in cubic feet per second } \\
\hline $\begin{array}{l}1 \\
2 \\
3 \\
4 \\
5\end{array}$ & $\begin{array}{l}13 \\
12 \\
11 \\
11 \\
11\end{array}$ & $\begin{array}{l}8.5 \\
9.1 \\
8.6 \\
7.9 \\
6.8\end{array}$ & $\begin{array}{r}15 \\
14 \\
13 \\
9.0 \\
9.7\end{array}$ & $\begin{array}{l}5.7 \\
6.0 \\
6.2 \\
6.3 \\
5.2\end{array}$ & $\begin{array}{l}50 \\
37 \\
44 \\
24 \\
16\end{array}$ & $\begin{array}{r}160 \\
190 \\
70 \\
45 \\
25\end{array}$ & $\begin{array}{l}45 \\
42 \\
99 \\
79 \\
65\end{array}$ & $\begin{array}{l}17 \\
16 \\
16 \\
15 \\
14\end{array}$ & $\begin{array}{l}6.0 \\
6.4 \\
6.0 \\
5.6 \\
5.2\end{array}$ & $\begin{array}{l}2.7 \\
2.7 \\
2.6 \\
2.4 \\
2.1\end{array}$ & $\begin{array}{l}.94 \\
.64 \\
.54 \\
.56 \\
1.4\end{array}$ & $\begin{array}{l}.11 \\
.05 \\
.03 \\
.05 \\
.08\end{array}$ \\
\hline $\begin{array}{r}6 \\
7 \\
8 \\
9 \\
10\end{array}$ & $\begin{array}{c}11 \\
10 \\
10 \\
10 \\
9.7\end{array}$ & $\begin{array}{l}6.4 \\
7.2 \\
9.3 \\
8.2 \\
7.1\end{array}$ & $\begin{array}{l}11 \\
12 \\
12 \\
20 \\
21\end{array}$ & $\begin{array}{l}4.8 \\
6.3 \\
7.4 \\
6.6 \\
5.8\end{array}$ & $\begin{array}{c}13 \\
9.9 \\
9.6 \\
9.4 \\
7.4\end{array}$ & $\begin{array}{r}50 \\
100 \\
130 \\
110 \\
70\end{array}$ & $\begin{array}{l}56 \\
46 \\
41 \\
37 \\
35\end{array}$ & $\begin{array}{l}13 \\
13 \\
16 \\
45 \\
27\end{array}$ & $\begin{array}{l}4.9 \\
4.7 \\
4.6 \\
4.1 \\
4.0\end{array}$ & $\begin{array}{l}1.9 \\
1.8 \\
1.8 \\
1.9 \\
3.4\end{array}$ & $\begin{array}{l}1.6 \\
.98 \\
2.4 \\
3.5 \\
2.3\end{array}$ & $\begin{array}{l}.10 \\
.08 \\
.07 \\
.06 \\
.06\end{array}$ \\
\hline $\begin{array}{l}11 \\
12 \\
13 \\
14 \\
15\end{array}$ & $\begin{array}{l}9.3 \\
9.4 \\
9.8 \\
9.4 \\
9.5\end{array}$ & $\begin{array}{l}7.0 \\
7.3 \\
7.7 \\
7.4 \\
7.1\end{array}$ & $\begin{array}{l}18 \\
17 \\
15 \\
10 \\
7.3\end{array}$ & $\begin{array}{l}6.0 \\
7.0 \\
6.6 \\
6.2 \\
7.0\end{array}$ & $\begin{array}{l}6.8 \\
6.4 \\
6.2 \\
6.4 \\
6.2\end{array}$ & $\begin{array}{l}45 \\
36 \\
32 \\
27 \\
35\end{array}$ & $\begin{array}{l}33 \\
32 \\
30 \\
28 \\
26\end{array}$ & $\begin{array}{l}20 \\
19 \\
17 \\
15 \\
15\end{array}$ & $\begin{array}{l}4.0 \\
3.9 \\
3.6 \\
2.9 \\
3.0\end{array}$ & $\begin{array}{l}4.8 \\
3.1 \\
2.6 \\
2.5 \\
2.6\end{array}$ & $\begin{array}{c}1.4 \\
1.1 \\
.83 \\
.73 \\
.80\end{array}$ & $\begin{array}{l}.05 \\
.04 \\
.04 \\
.03 \\
.03\end{array}$ \\
\hline $\begin{array}{l}16 \\
17 \\
18 \\
19 \\
20\end{array}$ & $\begin{array}{c}9.7 \\
12 \\
11 \\
9.3 \\
8.6\end{array}$ & $\begin{array}{c}8.1 \\
12 \\
18 \\
11 \\
9.1\end{array}$ & $\begin{array}{l}9.0 \\
12 \\
11 \\
12 \\
15\end{array}$ & $\begin{array}{l}7.2 \\
7.0 \\
6.8 \\
6.7 \\
6.6\end{array}$ & $\begin{array}{l}6.2 \\
6.4 \\
6.4 \\
6.2 \\
6.0\end{array}$ & $\begin{array}{l}33 \\
37 \\
36 \\
35 \\
34\end{array}$ & $\begin{array}{l}25 \\
23 \\
22 \\
20 \\
21\end{array}$ & $\begin{array}{l}14 \\
13 \\
12 \\
11 \\
11\end{array}$ & $\begin{array}{l}2.9 \\
3.0 \\
3.3 \\
3.7 \\
3.7\end{array}$ & $\begin{array}{l}2.2 \\
2.5 \\
2.6 \\
1.9 \\
1.9\end{array}$ & $\begin{array}{l}.65 \\
.55 \\
.39 \\
.34 \\
.31\end{array}$ & $\begin{array}{c}.03 \\
.02 \\
.02 \\
6.1 \\
15\end{array}$ \\
\hline $\begin{array}{l}21 \\
22 \\
23 \\
24 \\
25\end{array}$ & $\begin{array}{l}8.8 \\
8.7 \\
8.7 \\
8.4 \\
8.1\end{array}$ & $\begin{array}{l}8.1 \\
9.1 \\
9.5 \\
9.0 \\
8.5\end{array}$ & $\begin{array}{l}13 \\
12 \\
11 \\
9.6 \\
8.6\end{array}$ & $\begin{array}{l}6.4 \\
6.4 \\
6.0 \\
6.0 \\
6.3\end{array}$ & $\begin{array}{l}5.6 \\
5.8 \\
5.8 \\
5.4 \\
5.5\end{array}$ & $\begin{array}{l}30 \\
30 \\
30 \\
30 \\
75\end{array}$ & $\begin{array}{l}20 \\
21 \\
22 \\
22 \\
19\end{array}$ & $\begin{array}{r}11 \\
9.9 \\
9.0 \\
8.3 \\
7.9\end{array}$ & $\begin{array}{l}3.3 \\
2.9 \\
2.5 \\
2.4 \\
2.6\end{array}$ & $\begin{array}{l}2.2 \\
2.0 \\
1.8 \\
1.7 \\
1.8\end{array}$ & $\begin{array}{c}.26 \\
.46 \\
.86 \\
1.2 \\
.72\end{array}$ & $\begin{array}{l}5.6 \\
25 \\
23 \\
6.9 \\
3.2\end{array}$ \\
\hline $\begin{array}{l}26 \\
27 \\
28 \\
29 \\
30 \\
31\end{array}$ & $\begin{array}{l}8.2 \\
8.1 \\
8.1 \\
7.9 \\
7.8 \\
7.6\end{array}$ & $\begin{array}{c}8.2 \\
7.9 \\
10 \\
30 \\
19\end{array}$ & $\begin{array}{l}7.8 \\
8.6 \\
7.6 \\
7.0 \\
9.6 \\
7.3\end{array}$ & $\begin{array}{r}5.5 \\
5.8 \\
6.2 \\
6.2 \\
10.2 \\
7.2\end{array}$ & $\begin{array}{r}5.6 \\
6.2 \\
8.4 \\
82\end{array}$ & $\begin{array}{l}53 \\
40 \\
45 \\
77 \\
60 \\
50\end{array}$ & $\begin{array}{l}18 \\
31 \\
25 \\
20 \\
18\end{array}$ & $\begin{array}{l}7.8 \\
7.8 \\
7.8 \\
7.1 \\
6.5 \\
6.3\end{array}$ & $\begin{array}{l}2.4 \\
2.1 \\
2.1 \\
2.3 \\
2.8\end{array}$ & $\begin{array}{l}1.8 \\
1.4 \\
1.4 \\
1.4 \\
1.3 \\
1.2\end{array}$ & $\begin{array}{l}.32 \\
.20 \\
.18 \\
.15 \\
.12 \\
.11\end{array}$ & $\begin{array}{c}2.0 \\
1.1 \\
.57 \\
.78 \\
.83\end{array}$ \\
\hline
\end{tabular}

Daily mean specific conductance, in microsiemens per centimeter at 25 degrees Celsius

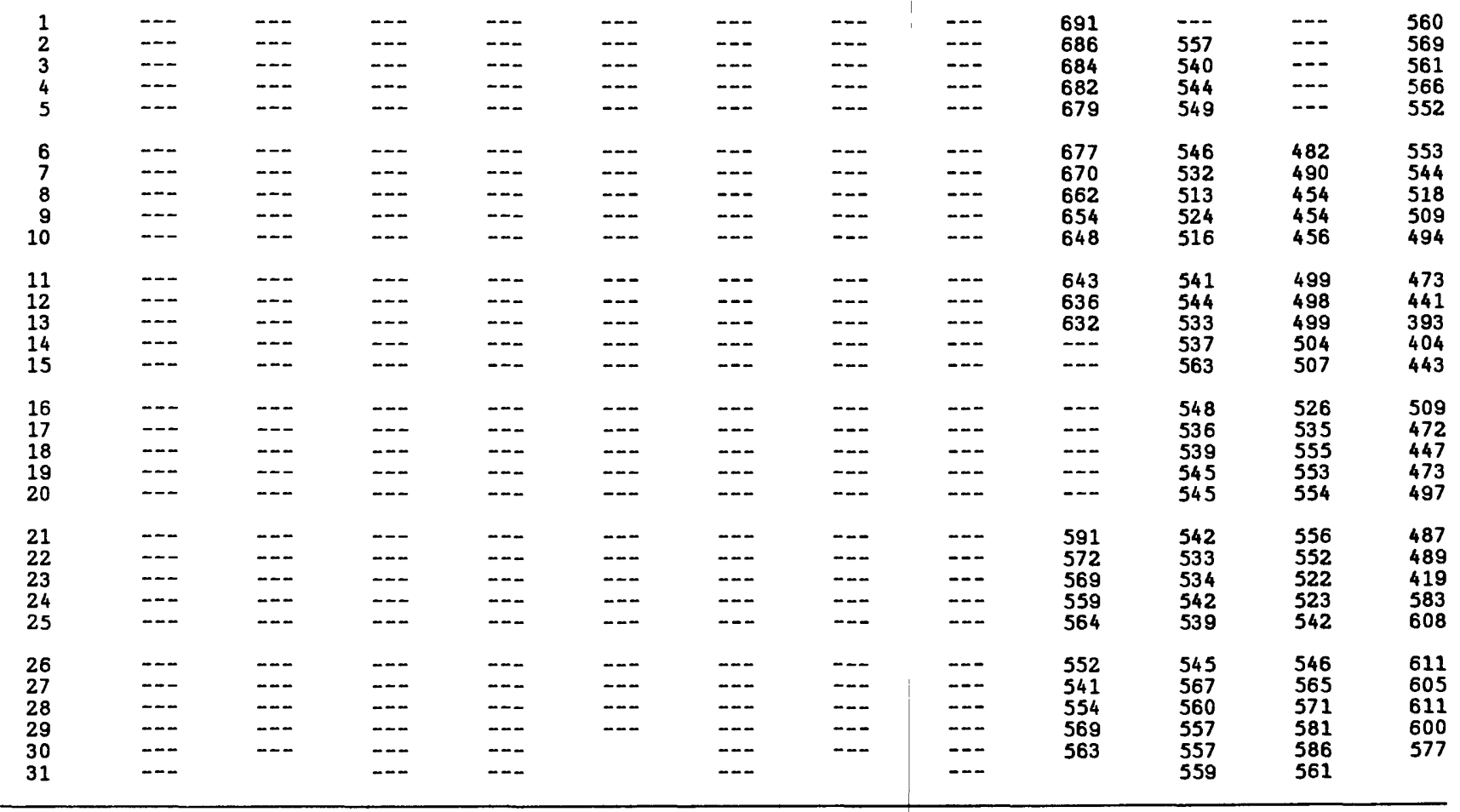



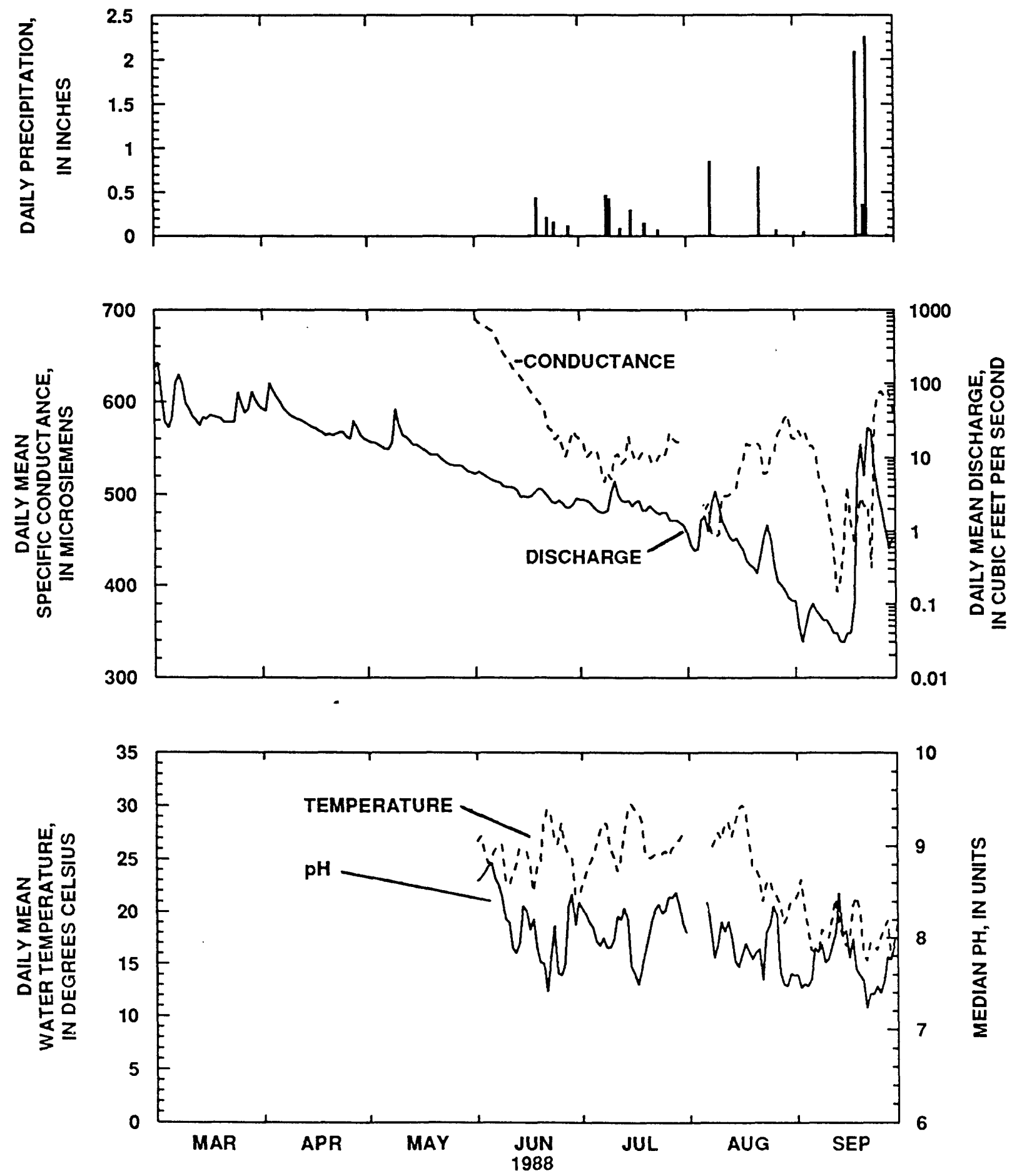

Figure 3.--Daily precipitation, daily mean specific conductance, discharge, water temperature, and daily median $\mathrm{pH}$ at site RC2, Roberts Creek above Saint 0laf, Iowa, March-September, 1988. 
Table 14.--Daily mean water temperature and median pH at site RC2, Roberts Creek above Saint Olaf lowa

Water year October 1987 to September 1988

[---, data not available to calculate mean or median values]

\begin{tabular}{|c|c|c|c|c|c|c|c|c|c|c|c|c|}
\hline Day & Oct & Nov & Dec & Jan & Feb & Mar & Apr & May & June & July & Aug & Sept \\
\hline & & & & ily & rater & perat & \multicolumn{6}{|c|}{ in degrees Celcius } \\
\hline $\begin{array}{l}1 \\
2 \\
3 \\
4 \\
5\end{array}$ & $\begin{array}{l}--- \\
--- \\
--- \\
--- \\
---\end{array}$ & $\begin{array}{l}--- \\
--- \\
--- \\
--- \\
---\end{array}$ & $\begin{array}{l}--- \\
--- \\
--- \\
--- \\
---\end{array}$ & $\begin{array}{l}--- \\
--- \\
--- \\
--- \\
---\end{array}$ & $\begin{array}{l}--- \\
--- \\
--- \\
--- \\
---\end{array}$ & $\begin{array}{l}--- \\
--- \\
--- \\
--- \\
---\end{array}$ & $\begin{array}{l}--- \\
--- \\
--- \\
--- \\
---\end{array}$ & $\begin{array}{l}--- \\
--- \\
--- \\
--- \\
---\end{array}$ & $\begin{array}{l}26.7 \\
27.2 \\
25.8 \\
24.6 \\
25.2\end{array}$ & $\begin{array}{r}--- \\
23.6 \\
24.5 \\
25.2 \\
26.2\end{array}$ & $\begin{array}{l}--- \\
--- \\
--- \\
--- \\
---\end{array}$ & $\begin{array}{l}21.6 \\
23.1 \\
20.8 \\
18.3 \\
16.3\end{array}$ \\
\hline $\begin{array}{r}6 \\
7 \\
8 \\
9 \\
10\end{array}$ & $\begin{array}{l}--- \\
--- \\
--- \\
--- \\
---\end{array}$ & $\begin{array}{l}--- \\
--- \\
--- \\
--- \\
---\end{array}$ & $\begin{array}{l}--- \\
--- \\
--- \\
--- \\
---\end{array}$ & $\begin{array}{l}--- \\
--- \\
--- \\
--- \\
---\end{array}$ & $\begin{array}{l}--- \\
--- \\
--- \\
--- \\
---\end{array}$ & $\begin{array}{l}--- \\
--- \\
--- \\
--- \\
---\end{array}$ & $\begin{array}{l}--- \\
--- \\
--- \\
--- \\
---\end{array}$ & $\begin{array}{l}--- \\
--- \\
--- \\
--- \\
---\end{array}$ & $\begin{array}{l}25.9 \\
26.3 \\
26.4 \\
23.0 \\
22.5\end{array}$ & $\begin{array}{l}27.5 \\
28.4 \\
28.3 \\
26.1 \\
25.3\end{array}$ & $\begin{array}{l}26.1 \\
25.9 \\
26.6 \\
27.6 \\
26.9\end{array}$ & $\begin{array}{l}16.2 \\
16.1 \\
18.3 \\
18.0 \\
17.5\end{array}$ \\
\hline $\begin{array}{l}11 \\
12 \\
13 \\
14 \\
15\end{array}$ & $\begin{array}{l}--- \\
--- \\
--- \\
--- \\
---\end{array}$ & $\begin{array}{l}--- \\
--- \\
--- \\
--- \\
---\end{array}$ & $\begin{array}{l}--- \\
--- \\
--- \\
--- \\
---\end{array}$ & $\begin{array}{l}--- \\
--- \\
--- \\
--- \\
---\end{array}$ & $\begin{array}{l}--- \\
--- \\
--- \\
--- \\
---\end{array}$ & $\begin{array}{l}--- \\
--- \\
--- \\
--- \\
---\end{array}$ & $\begin{array}{l}--- \\
--- \\
--- \\
--- \\
---\end{array}$ & $\begin{array}{l}--- \\
--- \\
--- \\
--- \\
---\end{array}$ & $\begin{array}{l}23.5 \\
24.5 \\
26.0 \\
26.0 \\
25.7\end{array}$ & $\begin{array}{l}23.9 \\
25.0 \\
27.2 \\
29.6 \\
30.2\end{array}$ & $\begin{array}{l}28.1 \\
28.6 \\
27.1 \\
28.3 \\
29.7\end{array}$ & $\begin{array}{l}19.4 \\
21.3 \\
18.9 \\
17.6 \\
16.7\end{array}$ \\
\hline $\begin{array}{l}16 \\
17 \\
18 \\
19 \\
20\end{array}$ & $\begin{array}{l}--- \\
--- \\
--- \\
--- \\
\end{array}$ & $\begin{array}{l}--- \\
--- \\
--- \\
--- \\
---\end{array}$ & $\begin{array}{l}--- \\
--- \\
--- \\
--- \\
--\end{array}$ & $\begin{array}{l}--- \\
--- \\
--- \\
--- \\
--\end{array}$ & $\begin{array}{l}--- \\
--- \\
--- \\
--- \\
---\end{array}$ & $\begin{array}{l}--- \\
--- \\
--- \\
--- \\
---\end{array}$ & $\begin{array}{l}--- \\
--- \\
--- \\
--- \\
---\end{array}$ & $\begin{array}{l}--- \\
--- \\
--- \\
--- \\
---\end{array}$ & $\begin{array}{l}24.5 \\
21.9 \\
24.2 \\
24.4 \\
28.5\end{array}$ & $\begin{array}{l}29.7 \\
29.3 \\
28.8 \\
25.8 \\
25.1\end{array}$ & $\begin{array}{l}30.1 \\
29.7 \\
26.9 \\
24.2 \\
24.1\end{array}$ & $\begin{array}{l}17.4 \\
20.6 \\
21.5 \\
20.3 \\
16.3\end{array}$ \\
\hline $\begin{array}{l}21 \\
22 \\
23 \\
24 \\
25\end{array}$ & $\begin{array}{l}--- \\
--- \\
--- \\
--- \\
---\end{array}$ & $\begin{array}{l}--- \\
--- \\
--- \\
--- \\
---\end{array}$ & $\begin{array}{l}--- \\
--- \\
--- \\
--- \\
---\end{array}$ & $\begin{array}{l}--- \\
--- \\
--- \\
--- \\
--\end{array}$ & $\begin{array}{l}--- \\
--- \\
--- \\
--- \\
---\end{array}$ & $\begin{array}{l}--- \\
--- \\
--- \\
--- \\
---\end{array}$ & $\begin{array}{l}--- \\
--- \\
--- \\
--- \\
---\end{array}$ & $\begin{array}{l}--- \\
--- \\
--- \\
--- \\
---\end{array}$ & $\begin{array}{l}29.7 \\
29.2 \\
27.0 \\
26.4 \\
28.4\end{array}$ & $\begin{array}{l}25.2 \\
25.5 \\
25.6 \\
25.5 \\
25.8\end{array}$ & $\begin{array}{l}23.3 \\
21.0 \\
22.7 \\
23.1 \\
21.7\end{array}$ & $\begin{array}{l}15.3 \\
17.0 \\
16.9 \\
16.3 \\
17.5\end{array}$ \\
\hline $\begin{array}{l}26 \\
27 \\
28 \\
29 \\
30 \\
31\end{array}$ & $\begin{array}{l}--- \\
--- \\
--- \\
--- \\
--- \\
---\end{array}$ & $\begin{array}{l}--- \\
--- \\
--- \\
--- \\
---\end{array}$ & $\begin{array}{l}--- \\
--- \\
--- \\
--- \\
--- \\
---\end{array}$ & $\begin{array}{l}--- \\
--- \\
--- \\
--- \\
--- \\
---\end{array}$ & $\begin{array}{l}--- \\
--- \\
--- \\
---\end{array}$ & $\begin{array}{l}--- \\
--- \\
--- \\
--- \\
--- \\
---\end{array}$ & $\begin{array}{l}--- \\
--- \\
--- \\
--- \\
---\end{array}$ & $\begin{array}{l}--- \\
--- \\
--- \\
--- \\
--- \\
---\end{array}$ & $\begin{array}{l}26.3 \\
25.5 \\
25.1 \\
21.7 \\
21.5\end{array}$ & $\begin{array}{l}25.3 \\
26.1 \\
26.7 \\
26.8 \\
27.4 \\
27.2\end{array}$ & $\begin{array}{l}21.5 \\
20.7 \\
18.9 \\
19.6 \\
21.0 \\
21.1\end{array}$ & $\begin{array}{l}18.4 \\
19.2 \\
15.5 \\
17.2 \\
19.1\end{array}$ \\
\hline
\end{tabular}

Daily median ph, in units

\begin{tabular}{|c|c|c|c|c|c|c|c|c|c|c|c|c|}
\hline $\begin{array}{l}1 \\
2 \\
3 \\
4 \\
5\end{array}$ & $\begin{array}{l}--- \\
--- \\
--- \\
--- \\
---\end{array}$ & $\begin{array}{l}--- \\
--- \\
--- \\
--- \\
---\end{array}$ & $\begin{array}{l}--- \\
--- \\
--- \\
--- \\
---\end{array}$ & $\begin{array}{l}--- \\
--- \\
--- \\
--- \\
---\end{array}$ & $\begin{array}{l}--- \\
--- \\
--- \\
--- \\
---\end{array}$ & $\begin{array}{l}--- \\
--- \\
--- \\
--- \\
---\end{array}$ & $\begin{array}{l}--- \\
--- \\
--- \\
--- \\
---\end{array}$ & $\begin{array}{l}--- \\
--- \\
--- \\
--- \\
---\end{array}$ & $\begin{array}{l}8.62 \\
8.66 \\
8.71 \\
8.78 \\
8.82\end{array}$ & $\begin{array}{l}--- \\
8.26 \\
8.17 \\
8.11 \\
7.96\end{array}$ & $\begin{array}{l}--- \\
--- \\
--- \\
--- \\
---\end{array}$ & $\begin{array}{l}7.60 \\
7.45 \\
7.50 \\
7.47 \\
7.55\end{array}$ \\
\hline $\begin{array}{r}6 \\
7 \\
8 \\
9 \\
10\end{array}$ & $\begin{array}{l}--- \\
--- \\
--- \\
--- \\
---\end{array}$ & $\begin{array}{l}--- \\
--- \\
--- \\
--- \\
---\end{array}$ & $\begin{array}{l}--- \\
--- \\
--- \\
--- \\
---\end{array}$ & $\begin{array}{l}--- \\
--- \\
--- \\
--- \\
---\end{array}$ & $\begin{array}{l}--- \\
--- \\
--- \\
--- \\
---\end{array}$ & $\begin{array}{l}--- \\
--- \\
--- \\
--- \\
---\end{array}$ & $\begin{array}{l}--- \\
--- \\
--- \\
--- \\
---\end{array}$ & $\begin{array}{l}--- \\
--- \\
--- \\
--- \\
---\end{array}$ & $\begin{array}{l}8.65 \\
8.58 \\
8.45 \\
8.21 \\
8.17\end{array}$ & $\begin{array}{l}7.91 \\
8.00 \\
7.89 \\
7.89 \\
7.98\end{array}$ & $\begin{array}{l}8.39 \\
8.11 \\
7.78 \\
7.92 \\
8.18\end{array}$ & $\begin{array}{l}7.89 \\
7.84 \\
7.93 \\
7.73 \\
7.77\end{array}$ \\
\hline $\begin{array}{l}11 \\
12 \\
13 \\
14 \\
15\end{array}$ & $\begin{array}{l}--- \\
--- \\
--- \\
---\end{array}$ & $\begin{array}{l}--- \\
--- \\
--- \\
--- \\
---\end{array}$ & $\begin{array}{l}--- \\
--- \\
--- \\
--- \\
---\end{array}$ & $\begin{array}{l}--- \\
--- \\
--- \\
--- \\
---\end{array}$ & $\begin{array}{l}--- \\
--- \\
--- \\
--- \\
---\end{array}$ & $\begin{array}{l}--- \\
--- \\
--- \\
--- \\
---\end{array}$ & $\begin{array}{l}--- \\
--- \\
--- \\
--- \\
---\end{array}$ & $\begin{array}{l}--- \\
--- \\
--- \\
--- \\
---\end{array}$ & $\begin{array}{l}7.89 \\
7.83 \\
7.94 \\
8.35 \\
8.30\end{array}$ & $\begin{array}{l}8.24 \\
8.20 \\
8.33 \\
8.20 \\
7.68\end{array}$ & $\begin{array}{l}8.07 \\
8.18 \\
8.01 \\
7.75 \\
7.68\end{array}$ & $\begin{array}{l}7.91 \\
8.05 \\
8.49 \\
8.03 \\
8.08\end{array}$ \\
\hline $\begin{array}{l}16 \\
17 \\
18 \\
19 \\
20\end{array}$ & $\begin{array}{l}--- \\
--- \\
--- \\
---\end{array}$ & $\begin{array}{l}--- \\
--- \\
--- \\
--- \\
---\end{array}$ & $\begin{array}{l}--- \\
--- \\
--- \\
--- \\
---\end{array}$ & $\begin{array}{l}--- \\
--- \\
--- \\
--- \\
---\end{array}$ & $\begin{array}{l}--- \\
--- \\
--- \\
--- \\
---\end{array}$ & $\begin{array}{l}--- \\
--- \\
--- \\
--- \\
---\end{array}$ & $\begin{array}{l}--- \\
--- \\
--- \\
--- \\
---\end{array}$ & $\begin{array}{l}--- \\
--- \\
--- \\
--- \\
---\end{array}$ & $\begin{array}{l}8.09 \\
8.21 \\
7.89 \\
7.73 \\
7.72\end{array}$ & $\begin{array}{r}7.60 \\
7.49 \\
- \\
- \\
8.01\end{array}$ & $\begin{array}{l}7.81 \\
7.94 \\
7.85 \\
7.77 \\
7.84\end{array}$ & $\begin{array}{l}7.78 \\
7.99 \\
7.65 \\
7.59 \\
7.53\end{array}$ \\
\hline $\begin{array}{l}21 \\
22 \\
23 \\
24 \\
25\end{array}$ & $\begin{array}{l}--- \\
--- \\
--- \\
--- \\
--\end{array}$ & $\begin{array}{l}--- \\
--- \\
--- \\
--- \\
---\end{array}$ & $\begin{array}{l}--- \\
--- \\
--- \\
--- \\
---\end{array}$ & $\begin{array}{l}--- \\
--- \\
--- \\
--- \\
---\end{array}$ & $\begin{array}{l}--- \\
--- \\
--- \\
--- \\
---\end{array}$ & $\begin{array}{l}--- \\
--- \\
--- \\
--- \\
---\end{array}$ & $\begin{array}{l}--- \\
--- \\
--- \\
--- \\
---\end{array}$ & $\begin{array}{l}--- \\
--- \\
--- \\
--- \\
---\end{array}$ & $\begin{array}{l}7.41 \\
-.- \\
8.13 \\
7.61 \\
7.59\end{array}$ & $\begin{array}{l}8.20 \\
8.31 \\
8.37 \\
8.27 \\
8.30\end{array}$ & $\begin{array}{l}7.88 \\
7.54 \\
8.06 \\
8.15 \\
8.35\end{array}$ & $\begin{array}{l}7.23 \\
7.39 \\
7.38 \\
7.47 \\
7.39\end{array}$ \\
\hline $\begin{array}{l}26 \\
27 \\
28 \\
29 \\
30 \\
31\end{array}$ & $\begin{array}{l}--- \\
--- \\
--- \\
--- \\
--- \\
---\end{array}$ & $\begin{array}{l}--- \\
--- \\
--- \\
--- \\
--\end{array}$ & $\begin{array}{l}--- \\
--- \\
--- \\
--- \\
--- \\
---\end{array}$ & $\begin{array}{l}--- \\
--- \\
--- \\
--- \\
--- \\
---\end{array}$ & $\begin{array}{l}--- \\
--- \\
--- \\
---\end{array}$ & $\begin{array}{l}--- \\
--- \\
--- \\
--- \\
--- \\
---\end{array}$ & $\begin{array}{l}--- \\
--- \\
--- \\
--- \\
---\end{array}$ & $\begin{array}{l}--- \\
--- \\
--- \\
--- \\
--- \\
---\end{array}$ & $\begin{array}{l}7.71 \\
8.34 \\
8.48 \\
8.14 \\
8.39\end{array}$ & $\begin{array}{l}8.45 \\
8.44 \\
8.50 \\
8.36 \\
8.16 \\
8.06\end{array}$ & $\begin{array}{l}8.25 \\
7.62 \\
7.49 \\
7.47 \\
7.61 \\
7.59\end{array}$ & $\begin{array}{r}7.53 \\
7.79 \\
7.76 \\
7.91 \\
-\end{array}$ \\
\hline
\end{tabular}


Table 15. - Instantaneous discharge and suspended sediment concentrations Ift site RC2, Roberts creek above Saint Olaf, lowa

[ $\mathrm{ft}^{3} / \mathrm{s}$, cublc feet per second; mg/L, milligrams per liter]

\begin{tabular}{|c|c|c|c|c|c|c|c|}
\hline Date & Time & $\begin{array}{l}\text { Instan- } \\
\text { taneous } \\
\text { disgharge } \\
\left(\mathrm{ft}^{3} / \mathrm{s}\right)\end{array}$ & $\begin{array}{l}\text { Suspended } \\
\text { sediment } \\
(\mathrm{mg} / \mathrm{L})\end{array}$ & Date & Time & $\begin{array}{l}\text { Instan- } \\
\text { taneous } \\
\text { discharge } \\
\left(\mathrm{ft}^{3} / \mathrm{s}\right)\end{array}$ & $\begin{array}{l}\text { Suspended } \\
\text { sediment } \\
(\mathrm{mg} / \mathrm{L})\end{array}$ \\
\hline $\begin{array}{l}3-08-88 \\
3-23-88 \\
3-23-88 \\
3-25-88 \\
3-29-88\end{array}$ & $\begin{array}{l}1320 \\
1115 \\
1415 \\
1330 \\
1030\end{array}$ & $\begin{array}{r}232 \\
31 \\
31 \\
105 \\
80\end{array}$ & $\begin{array}{r}458 \\
60 \\
40 \\
458 \\
145\end{array}$ & $\begin{array}{l}6-25-88 \\
6-26-88 \\
6-28-88 \\
6-29-88 \\
7-01-88\end{array}$ & $\begin{array}{l}1635 \\
1620 \\
1645 \\
1645 \\
1615\end{array}$ & $\begin{array}{l}2.7 \\
2.4 \\
2.0 \\
2.4 \\
2.4\end{array}$ & $\begin{array}{r}29 \\
39 \\
34 \\
80 \\
201\end{array}$ \\
\hline $\begin{array}{l}3-31-88 \\
4-13-88 \\
4-14-88 \\
4-15-88 \\
4-16-88\end{array}$ & $\begin{array}{l}1330 \\
1630 \\
1645 \\
1710 \\
1645\end{array}$ & $\begin{array}{l}50 \\
30 \\
28 \\
26 \\
25\end{array}$ & $\begin{array}{l}42 \\
54 \\
70 \\
72 \\
69\end{array}$ & $\begin{array}{l}7-03-88 \\
7-04-88 \\
7-06-88 \\
7-07-88 \\
7-08-88\end{array}$ & $\begin{array}{l}1640 \\
1640 \\
1840 \\
1645 \\
1640\end{array}$ & $\begin{array}{l}2.7 \\
2.4 \\
1.9 \\
1.7 \\
1.8\end{array}$ & $\begin{array}{r}51 \\
47 \\
68 \\
64 \\
101\end{array}$ \\
\hline $\begin{array}{l}4-17-88 \\
4-19-88 \\
4-20-88 \\
4-21-88 \\
4-23-88\end{array}$ & $\begin{array}{l}1645 \\
1635 \\
1655 \\
1645 \\
1305\end{array}$ & $\begin{array}{l}22 \\
21 \\
21 \\
21 \\
22\end{array}$ & $\begin{array}{l}70 \\
20 \\
49 \\
23 \\
5.8\end{array}$ & $\begin{array}{l}7-09-88 \\
7-10-88 \\
7-10-88 \\
7-10-88 \\
7-11-88\end{array}$ & $\begin{array}{l}1635 \\
1845 \\
2045 \\
2245 \\
0045\end{array}$ & $\begin{array}{l}2.0 \\
4.1 \\
4.1 \\
5.3 \\
5.7\end{array}$ & $\begin{array}{r}139 \\
11 \\
19 \\
71 \\
42\end{array}$ \\
\hline $\begin{array}{l}4-24-88 \\
4-25-88 \\
4-26-88 \\
4-27-88 \\
4-28-88\end{array}$ & $\begin{array}{l}1630 \\
1650 \\
1650 \\
1640 \\
1650\end{array}$ & $\begin{array}{l}21 \\
18 \\
20 \\
37 \\
22\end{array}$ & $\begin{array}{l}4.2 \\
18 \\
39 \\
57 \\
20\end{array}$ & $\begin{array}{l}7-11-88 \\
7-11-88 \\
7-11-88 \\
7-11-88 \\
7-11-88\end{array}$ & $\begin{array}{l}0245 \\
0445 \\
0645 \\
0845 \\
1045\end{array}$ & $\begin{array}{l}5.7 \\
5.7 \\
5.3 \\
5.1 \\
4.9\end{array}$ & $\begin{array}{r}42 \\
112 \\
30 \\
12 \\
13\end{array}$ \\
\hline $\begin{array}{l}4-29-88 \\
5-01-88 \\
5-03-88 \\
5-04-88 \\
5-06-88\end{array}$ & $\begin{array}{l}1655 \\
1705 \\
1640 \\
1455 \\
1600\end{array}$ & $\begin{array}{l}19 \\
17 \\
16 \\
15 \\
13\end{array}$ & $\begin{array}{l}13 \\
24 \\
24 \\
41 \\
28\end{array}$ & $\begin{array}{l}7-12-88 \\
7-13-88 \\
7-15-88 \\
7-16-88 \\
7-17-88\end{array}$ & $\begin{array}{l}1641 \\
1720 \\
1505 \\
1530 \\
1705\end{array}$ & $\begin{array}{l}2.8 \\
2.5 \\
2.8 \\
2.4 \\
2.8\end{array}$ & $\begin{array}{r}21 \\
27 \\
52 \\
142 \\
28\end{array}$ \\
\hline $\begin{array}{l}5-07-88 \\
5-08-88 \\
5-10-88 \\
5-11-88 \\
5-13-88\end{array}$ & $\begin{array}{l}1650 \\
1750 \\
1640 \\
1635 \\
1710\end{array}$ & $\begin{array}{l}14 \\
19 \\
25 \\
20 \\
16\end{array}$ & $\begin{array}{l}29 \\
29 \\
46 \\
16 \\
51\end{array}$ & $\begin{array}{l}7-19-88 \\
7-20-88 \\
7-21-88 \\
7-22-88 \\
7-23-88\end{array}$ & $\begin{array}{l}1720 \\
1640 \\
1640 \\
1635 \\
1635\end{array}$ & $\begin{array}{l}1.8 \\
1.8 \\
2.1 \\
1.9 \\
1.7\end{array}$ & $\begin{array}{r}170 \\
83 \\
31 \\
60 \\
37\end{array}$ \\
\hline $\begin{array}{l}5-14-88 \\
5-15-88 \\
5-17-88 \\
5-18-88 \\
5-20-88\end{array}$ & $\begin{array}{l}1640 \\
1605 \\
1645 \\
1630 \\
1930\end{array}$ & $\begin{array}{l}15 \\
15 \\
13 \\
12 \\
11\end{array}$ & $\begin{array}{l}66 \\
16 \\
24 \\
35 \\
70\end{array}$ & $\begin{array}{l}7-24-88 \\
7-25-88 \\
7-26-88 \\
7-29-88 \\
8-01-88\end{array}$ & $\begin{array}{l}1630 \\
1828 \\
1753 \\
1100 \\
1115\end{array}$ & $\begin{array}{r}1.7 \\
1.7 \\
1.8 \\
1.4 \\
.82\end{array}$ & $\begin{array}{r}52 \\
176 \\
40 \\
12 \\
44\end{array}$ \\
\hline $\begin{array}{l}5-21-88 \\
5-22-88 \\
5-24-88 \\
5-25-88 \\
5-27-88\end{array}$ & $\begin{array}{l}1835 \\
1840 \\
1815 \\
1830 \\
1825\end{array}$ & $\begin{array}{r}11 \\
9.6 \\
8.2 \\
8.2 \\
7.9\end{array}$ & $\begin{array}{l}46 \\
62 \\
49 \\
83 \\
79\end{array}$ & $\begin{array}{l}8-05-88 \\
8-08-88 \\
8-09-88 \\
8-10-88 \\
8-10-88\end{array}$ & $\begin{array}{r}750 \\
1832 \\
1842 \\
1330 \\
1746\end{array}$ & $\begin{array}{r}.97 \\
1.2 \\
2.5 \\
2.2 \\
1.8\end{array}$ & $\begin{array}{l}23 \\
84 \\
75 \\
24 \\
70\end{array}$ \\
\hline $\begin{array}{l}5-28-88 \\
5-31-88 \\
6-01-88 \\
6-02-88 \\
6-04-88\end{array}$ & $\begin{array}{l}1820 \\
1820 \\
1820 \\
1150 \\
1720\end{array}$ & $\begin{array}{l}7.9 \\
6.2 \\
6.0 \\
6.4 \\
5.3\end{array}$ & $\begin{array}{l}50 \\
81 \\
81 \\
93 \\
58\end{array}$ & $\begin{array}{l}8-11-88 \\
8-12-88 \\
8-13-88 \\
8-15-88 \\
8-16-88\end{array}$ & $\begin{array}{l}1740 \\
1837 \\
1505 \\
1747 \\
1100\end{array}$ & $\begin{array}{r}1.2 \\
.97 \\
.89 \\
.75 \\
.54\end{array}$ & $\begin{array}{c}39 \\
56 \\
43 \\
62 \\
9.9\end{array}$ \\
\hline $\begin{array}{l}6-05-88 \\
6-07-88 \\
6-10-88 \\
6-11-88 \\
6-12-88\end{array}$ & $\begin{array}{l}1650 \\
1645 \\
1745 \\
1800 \\
1655\end{array}$ & $\begin{array}{l}4.9 \\
4.3 \\
3.9 \\
3.9 \\
3.7\end{array}$ & $\begin{array}{l}50 \\
28 \\
31 \\
50 \\
35\end{array}$ & $\begin{array}{l}8-17-88 \\
8-19-88 \\
8-20-88 \\
8-21-88 \\
8-22-88\end{array}$ & $\begin{array}{l}1747 \\
1632 \\
1810 \\
1745 \\
1315\end{array}$ & $\begin{array}{l}.48 \\
.35 \\
.30 \\
.25 \\
.60\end{array}$ & $\begin{array}{r}75 \\
149 \\
118 \\
80 \\
36\end{array}$ \\
\hline $\begin{array}{l}6-14-88 \\
6-15-88 \\
6-17-88 \\
6-18-88 \\
6-19-88\end{array}$ & $\begin{array}{l}1720 \\
1825 \\
1650 \\
1645 \\
1645\end{array}$ & $\begin{array}{l}2.4 \\
2.9 \\
3.1 \\
3.4 \\
3.9\end{array}$ & $\begin{array}{r}69 \\
27 \\
22 \\
29 \\
110\end{array}$ & $\begin{array}{l}8-22-88 \\
8-23-88 \\
8-24-88 \\
8-25-88 \\
8-25-88\end{array}$ & $\begin{array}{l}1637 \\
1750 \\
1812 \\
1222 \\
1640\end{array}$ & $\begin{array}{l}.60 \\
.75 \\
1.3 \\
.67 \\
.41\end{array}$ & $\begin{array}{r}133 \\
70 \\
44 \\
83 \\
30\end{array}$ \\
\hline $\begin{array}{l}6-21-88 \\
6-22-88 \\
6-23-88 \\
6-24-88 \\
6-24-88\end{array}$ & $\begin{array}{r}1635 \\
1640 \\
1600 \\
830 \\
1930\end{array}$ & $\begin{array}{l}2.9 \\
3.1 \\
2.4 \\
2.3 \\
2.7\end{array}$ & $\begin{array}{r}26 \\
32 \\
20 \\
165 \\
54\end{array}$ & $\begin{array}{l}8-26-88 \\
8-28-88 \\
8-29-88 \\
8-30-88 \\
8-31-88\end{array}$ & $\begin{array}{l}1615 \\
1625 \\
1645 \\
1635 \\
1630\end{array}$ & $\begin{array}{l}.30 \\
.12 \\
.01 \\
.17 \\
.12\end{array}$ & $\begin{array}{r}28 \\
90 \\
115 \\
75 \\
64\end{array}$ \\
\hline
\end{tabular}


Table 15.--Instantaneous discharge and suspended sediment concentrations at sjte RC2, Roberts Creek above saint olaf, Iowa--continued

\begin{tabular}{|c|c|c|c|c|c|c|c|}
\hline Date & Time & $\begin{array}{l}\text { Instan- } \\
\text { taneous } \\
\text { disçharge } \\
\text { (ft } 3 / \mathrm{s})\end{array}$ & $\begin{array}{l}\text { Suspended } \\
\text { s ediment } \\
\text { (mg/L) }\end{array}$ & Date & Time & $\begin{array}{l}\text { Instan- } \\
\text { taneous } \\
\text { discharge } \\
\left(\mathrm{ft}^{3} / \mathrm{s}\right)\end{array}$ & $\begin{array}{l}\text { Suspended } \\
\text { sediment } \\
(\mathrm{mg} / \mathrm{L})\end{array}$ \\
\hline $\begin{array}{l}9-01-88 \\
9-02-88 \\
9-04-88 \\
9-05-88 \\
9-06-88\end{array}$ & $\begin{array}{l}1630 \\
1630 \\
1635 \\
1630 \\
1000\end{array}$ & $\begin{array}{l}.12 \\
.04 \\
.04 \\
.08 \\
.08\end{array}$ & $\begin{array}{r}103 \\
62 \\
244 \\
106 \\
29\end{array}$ & $\begin{array}{l}9-21-88 \\
9-21-88 \\
9-21-88 \\
9-22-88 \\
9-22-88\end{array}$ & $\begin{array}{r}1100 \\
1300 \\
1625 \\
715 \\
920\end{array}$ & $\begin{array}{l}5.3 \\
4.9 \\
4.1 \\
30 \\
12\end{array}$ & $\begin{array}{r}30 \\
22 \\
84 \\
152 \\
263\end{array}$ \\
\hline $\begin{array}{l}9-06-88 \\
9-07-88 \\
9-09-88 \\
9-10-88 \\
9-11-88\end{array}$ & $\begin{array}{l}1635 \\
1625 \\
1625 \\
1630 \\
1700\end{array}$ & $\begin{array}{l}.12 \\
.17 \\
.12 \\
.17 \\
.12\end{array}$ & $\begin{array}{r}47 \\
129 \\
135 \\
181 \\
113\end{array}$ & $\begin{array}{l}9-22-88 \\
9-22-88 \\
9-22-88 \\
9-22-88 \\
9-22-88\end{array}$ & $\begin{array}{l}1115 \\
1145 \\
1215 \\
1245 \\
1307\end{array}$ & $\begin{array}{l}15 \\
20 \\
22 \\
24 \\
39\end{array}$ & $\begin{array}{l}256 \\
347 \\
442 \\
418 \\
683\end{array}$ \\
\hline $\begin{array}{l}9-12-88 \\
9-16-88 \\
9-17-88 \\
9-19-88 \\
9-19-88\end{array}$ & $\begin{array}{r}1630 \\
1625 \\
1700 \\
945 \\
1635\end{array}$ & $\begin{array}{l}.04 \\
.12 \\
.17 \\
1.9 \\
1.3\end{array}$ & $\begin{array}{r}120 \\
220 \\
182 \\
90 \\
109\end{array}$ & $\begin{array}{l}9-22-88 \\
9-22-88 \\
9-22-88 \\
9-22-88 \\
9-22-88\end{array}$ & $\begin{array}{l}1315 \\
1325 \\
1335 \\
1345 \\
1400\end{array}$ & $\begin{array}{l}52 \\
66 \\
76 \\
80 \\
83\end{array}$ & $\begin{array}{l}810 \\
692 \\
598 \\
556 \\
482\end{array}$ \\
\hline $\begin{array}{l}9-19-88 \\
9-19-88 \\
9-19-88 \\
9-20-88 \\
9-20-88\end{array}$ & $\begin{array}{r}1700 \\
1900 \\
2300 \\
100 \\
300\end{array}$ & $\begin{array}{l}2.2 \\
25 \\
14 \\
13 \\
12\end{array}$ & $\begin{array}{l}116 \\
333 \\
176 \\
244 \\
271\end{array}$ & $\begin{array}{l}9-22-88 \\
9-22-88 \\
9-22-88 \\
9-22-88 \\
9-22-88\end{array}$ & $\begin{array}{l}1415 \\
1445 \\
1515 \\
1545 \\
1615\end{array}$ & $\begin{array}{l}81 \\
73 \\
65 \\
57 \\
51\end{array}$ & $\begin{array}{l}407 \\
339 \\
275 \\
230 \\
266\end{array}$ \\
\hline $\begin{array}{l}9-20-88 \\
9-20-88 \\
9-20-88 \\
9-20-88 \\
9-20-88\end{array}$ & $\begin{array}{r}500 \\
700 \\
900 \\
935 \\
1100\end{array}$ & $\begin{array}{l}11 \\
14 \\
22 \\
22 \\
22\end{array}$ & $\begin{array}{l}298 \\
206 \\
164 \\
123 \\
163\end{array}$ & $\begin{array}{l}9-22-88 \\
9-22-88 \\
9-22-88 \\
9-22-88 \\
9-22-88\end{array}$ & $\begin{array}{l}1630 \\
1645 \\
1715 \\
1745 \\
1815\end{array}$ & $\begin{array}{l}48 \\
45 \\
40 \\
36 \\
32\end{array}$ & $\begin{array}{l}779 \\
231 \\
256 \\
280 \\
293\end{array}$ \\
\hline $\begin{array}{l}9-20-88 \\
9-20-88 \\
9-20-88 \\
9-20-88 \\
9-20-88\end{array}$ & $\begin{array}{l}1300 \\
1500 \\
1625 \\
1700 \\
1900\end{array}$ & $\begin{array}{l}21 \\
18 \\
16 \\
16 \\
13\end{array}$ & $\begin{array}{l}118 \\
123 \\
227 \\
127 \\
138\end{array}$ & $\begin{array}{l}9-22-88 \\
9-22-88 \\
9-22-88 \\
9-22-88 \\
9-22-88\end{array}$ & $\begin{array}{l}1845 \\
1915 \\
1945 \\
2015 \\
2045\end{array}$ & $\begin{array}{l}27 \\
28 \\
27 \\
25 \\
23\end{array}$ & $\begin{array}{l}303 \\
311 \\
302 \\
279 \\
266\end{array}$ \\
\hline $\begin{array}{l}9-20-88 \\
9-20-88 \\
9-21-88 \\
9-21-88 \\
9-21-88\end{array}$ & $\begin{array}{r}2100 \\
2300 \\
100 \\
300 \\
500\end{array}$ & $\begin{array}{r}11 \\
9.6 \\
8.5 \\
7.9 \\
7.0\end{array}$ & $\begin{array}{r}148 \\
116 \\
99 \\
90 \\
74\end{array}$ & $\begin{array}{l}9-22-88 \\
9-22-88 \\
9-22-88 \\
9-22-88 \\
9-23-88\end{array}$ & $\begin{array}{l}2115 \\
2145 \\
2215 \\
2245 \\
1635\end{array}$ & $\begin{array}{l}23 \\
23 \\
23 \\
23 \\
17\end{array}$ & $\begin{array}{l}246 \\
234 \\
211 \\
213 \\
194\end{array}$ \\
\hline $\begin{array}{l}9-21-88 \\
9-21-88\end{array}$ & $\begin{array}{l}700 \\
900\end{array}$ & $\begin{array}{l}6.8 \\
6.0\end{array}$ & $\begin{array}{l}55 \\
56\end{array}$ & $\begin{array}{l}9-24-88 \\
9-26-88\end{array}$ & $\begin{array}{l}1625 \\
1820\end{array}$ & $\begin{array}{l}5.3 \\
1.8\end{array}$ & $\begin{array}{l}106 \\
106\end{array}$ \\
\hline
\end{tabular}




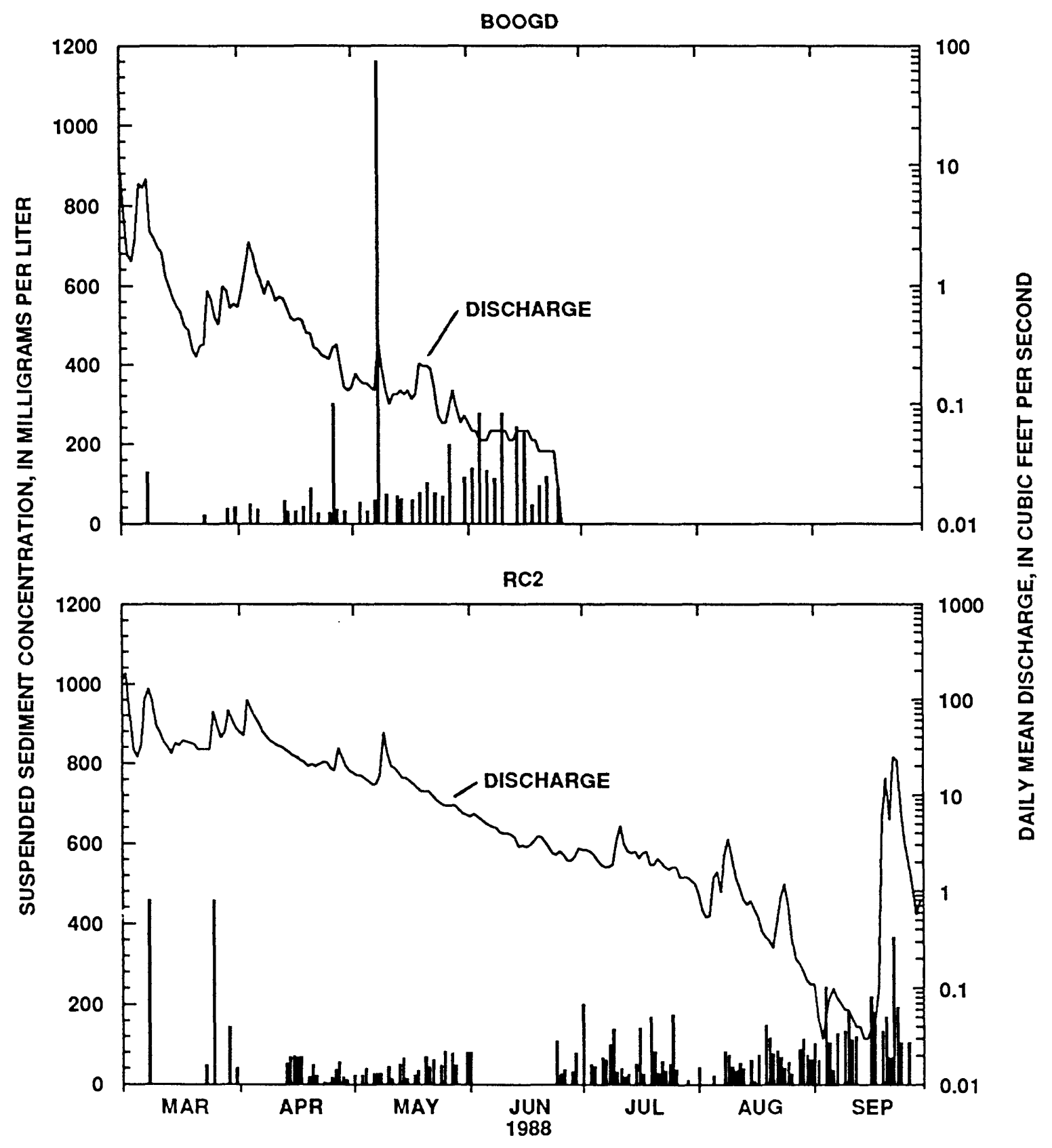

Figure 4.--Discharge and suspended sediment concentrations at sites BOOGD, Unnamed Creek near Luana, and RC2,

Roberts Creek above Saint Olaf, Iowa, March-September 1988. 
To define the diurnal variation in water quality at site RC2 during a period of low streamflow, water-quality samples were collected at three hour intervals from 0900 hours on June 23 to 0600 hours on June 24, 1988.

Concentrations of selected constituents are listed in table 16 and are shown in figure 5. Stream discharge varied slightly from 2.1 to $2.5 \mathrm{ft}^{3} / \mathrm{s}$.

Specific conductance was lowest, $540 \mu \mathrm{S} / \mathrm{cm}$, in the afternoon of June 23 and greatest, $600 \mu \mathrm{S} / \mathrm{cm}$, the following morning. Water temperature, $\mathrm{pH}$, and dissolved oxygen varied inversely in relation to specific conductance. Water temperature and $\mathrm{pH}$ reached maximum values during mid-afternoon and were at minimum values in mid-morning. The maximum $\mathrm{pH}$ and water temperature were 8.80 units and $31.3^{\circ} \mathrm{C}$, respectively and the minimum $\mathrm{pH}$ and water temperature were 7.34 units and $21.9{ }^{\circ} \mathrm{C}$, respectively. Dissolved-oxygen concentrations increased through the morning and early afternoon and peaked at $14.0 \mathrm{mg} / \mathrm{L}$ at mid-afternoon. Dissolved-oxygen concentrations are supersaturated at this point. Concentrations decrease during the late afternoon and evening and reached a minimum of $4.0 \mathrm{mg} / \mathrm{L}$ during the middle of the night.

Concentrations of most dissolved nutrients were constant during this 24-hour period (table 16). Nitrite plus nitrate concentrations were 0.5 to $0.6 \mathrm{mg} / \mathrm{L}$ as nitrogen. Ammonia was detected $(0.2 \mathrm{mg} / \mathrm{L}$ as nitrogen) only at 0600 hours on June 24. Ortho-phosphorus concentrations were constant at 0.3 $\mathrm{mg} / \mathrm{L}$. Organic nitrogen concentrations generally were lowest from 1200 hours to 1800 hours and greatest during the night.

Four samples for pesticide analyses were collected during the diurnal sampling. Two pesticides were detected (table 16). Atrazine was detected in all samples and cyanazine in one sample. The total recoverable atrazine concentration was $0.25 \mu \mathrm{g} / \mathrm{L}$ in one sample and $0.26 \mu \mathrm{g} / \mathrm{L}$ in the other three samples. The total recoverable cyanazine concentration was $0.12 \mu \mathrm{g} / \mathrm{L}$ at 1800 hours on June 23. 
Table 16.--Diurnal variation of selected physical and chemical 3 constituents at site RC2, Creek above St. Olaf, Iowa

$\mathrm{ft}^{3} / \mathrm{s}$, cubic feet per second; $\mu \mathrm{S} / \mathrm{cm}$, microsiemens per centimeter

$C$, degrees celsius; mg/L, milligrams per liter; N, nitrogen;

$P$, phosphorus; C, carbon; $\mu_{8} / L$, micrograms per liter;

- , data not collected]

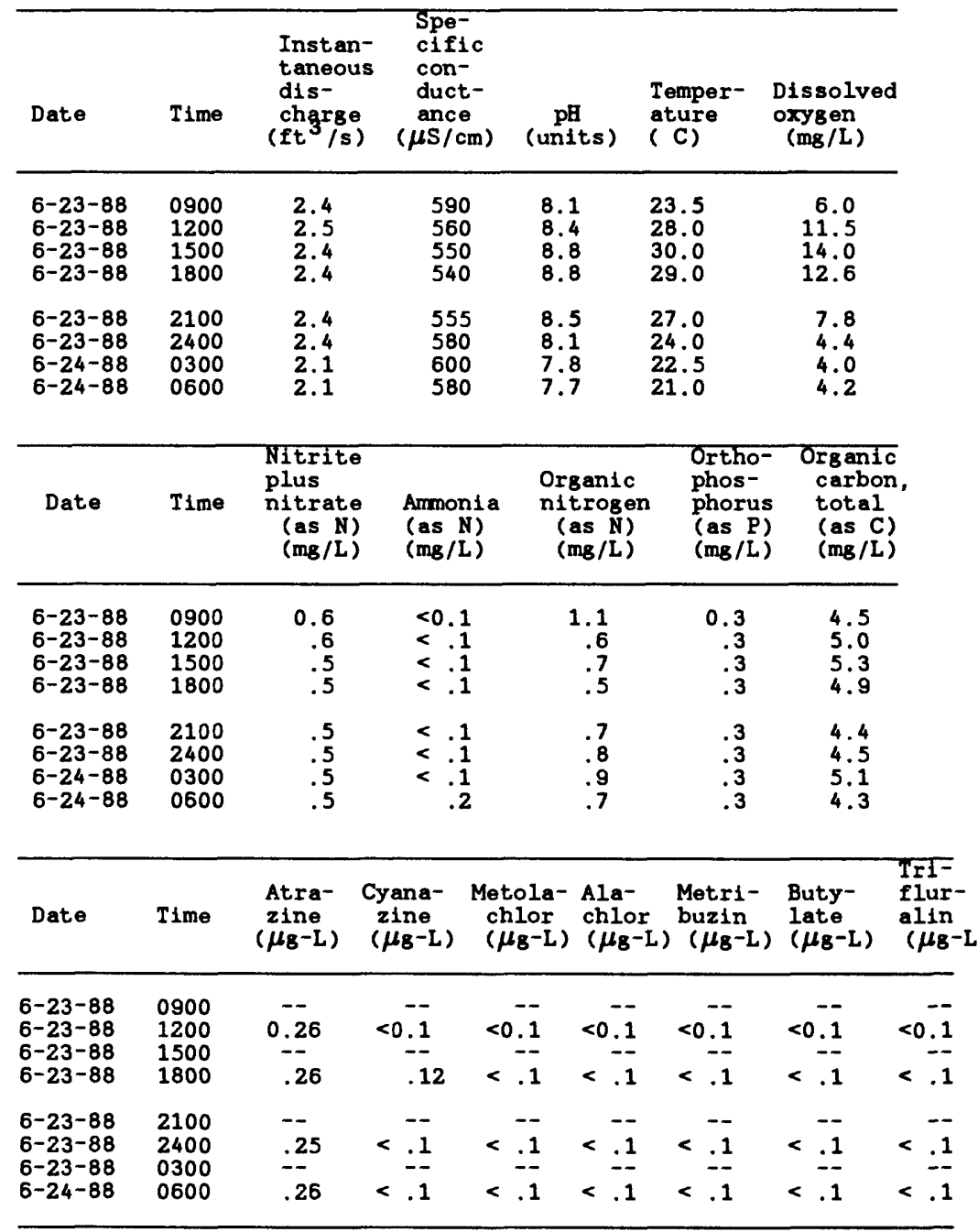



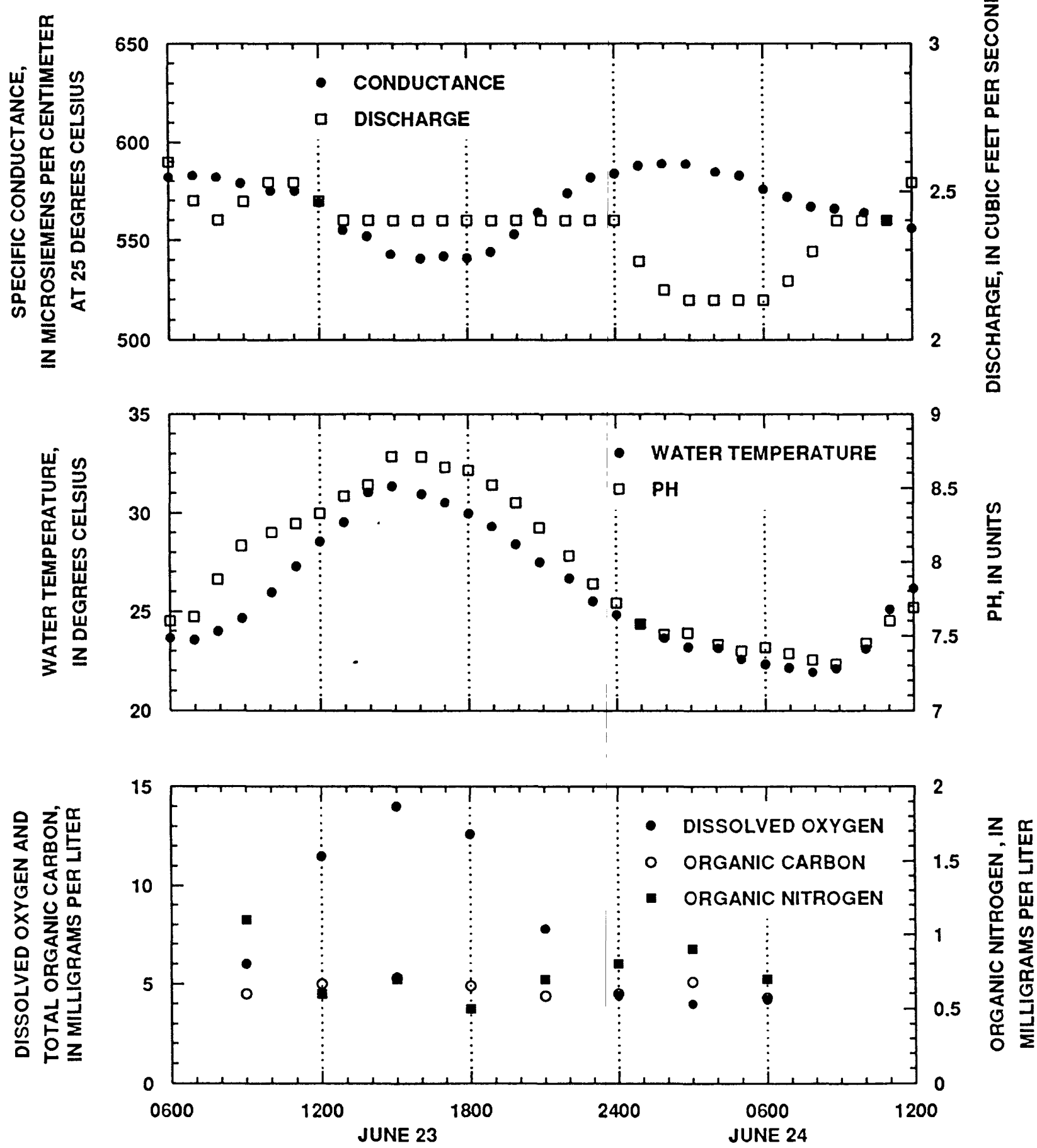

Figure 5.--Diurnal variation of selected physical and chemical constituents at site RC2, Roberts Creek above Saint Olaf, Iowa, June 23-24, 1988. 


\section{Ground Water}

Big Spring

Continuous water quality data, as daily mean and median values, collected at Big Spring are shown in tables 17 and 18 and in figure 6 . After installation of the monitoring equipment, it was determined that water quality varied from the top to the bottom of the spring pool. To obtain more representative values of water discharging from the spring, the water-quality-monitoring probes were lowered to within one foot of the bottom of the spring pool on May 4. The following discussion of water temperature, specific conductance, and $\mathrm{pH}$ deal only with values observed after May 4 . Daily mean specific conductance values ranged from $690 \mu \mathrm{S} / \mathrm{cm}$ on June 7 and 9 to $735 \mu \mathrm{S} / \mathrm{cm}$ on September 21 and 24. The maximum daily median $\mathrm{pH}$ was 7.10 units on May 5 and the minimum daily median pH was on 6.65 units on July 8 . Daily mean temperatures varied from 9.7 to $10.6{ }^{\circ} \mathrm{C}$ from May 5 to September 30 .

The predominant cations in solution were calcium and magnesium (table 9). Calcium concentrations ranged from 64 to $96 \mathrm{mg} / \mathrm{L}$ and magnesium concentrations ranged from 25 to $39 \mathrm{mg} / \mathrm{L}$. Sodium and potassium concentrations were less than $15 \mathrm{mg} / \mathrm{L}$. The predominant anion was bicarbonate. Bicarbonate concentrations ranged from 264 to $390 \mathrm{mg} / \mathrm{L}$. Sulfate concentrations ranged from 23 to $37 \mathrm{mg} / \mathrm{L}$ and chloride concentrations ranged from 14 to $24 \mathrm{mg} / \mathrm{L}$. Silica concentrations were $17 \mathrm{mg} / \mathrm{L}$ or less. Dissolved nitrate plus nitrite was the predominant nitrogen species in solution (table 10). Nitrate plus nitrite concentrations ranged from 7.5 to $11 \mathrm{mg} / \mathrm{L}$ as nitrogen. Two of eight samples had nitrate concentrations equal to or greater than the $10 \mathrm{mg} / \mathrm{L}$ maximum contaminant level (U.S. Environmental Protection Agency, 1986). Ammonia concentrations exceeded the detection limits in two samples. Organic nitrogen concentrations were less than the detection limits in three samples. The maximum organic nitrogen concentration in the remaining samples was $0.6 \mathrm{mg} / \mathrm{L}$ as nitrogen.

Atrazine was the only pesticide detected in Big Spring (table 11). Total recoverable atrazine concentrations ranged from less than the detection limit to $0.26 \mu \mathrm{g} / \mathrm{L}$ in a sample collected on May 4. Concentrations of cyanazine, metolachlor, alachlor, metribuzin, butylate, and trifluralin were less than the detection limit in all samples. 
Table 17. - - Daily mean specific conductance and water temperature at Big Springa

Water year October 1987 to September 1988

[Data before May 4 from a corresponding spring pool gage height of

approximately 8.5 feet, and after May 4 from approximately 10 feet; , data not available to calculate mean values]

\begin{tabular}{|c|c|c|c|c|c|c|c|c|c|c|c|c|}
\hline Day & Oct & Kov & Dec & Jan & Feb & Mar & Apr & May & June & JuIy & Aug & Sept \\
\hline & & ily m & peci & zono & $\theta$ & rosi & $s$ per & imet & \multicolumn{4}{|c|}{ degrees Celsius } \\
\hline $\begin{array}{l}1 \\
2 \\
3 \\
4 \\
5\end{array}$ & $\begin{array}{l}--- \\
--- \\
--- \\
---\end{array}$ & $\begin{array}{l}--- \\
--- \\
--- \\
---\end{array}$ & $\begin{array}{l}--- \\
--- \\
--- \\
--- \\
---\end{array}$ & $\begin{array}{l}--- \\
--- \\
--\end{array}$ & $\begin{array}{l}--- \\
--- \\
--- \\
---\end{array}$ & $\begin{array}{l}--- \\
--- \\
--- \\
---\end{array}$ & $\begin{array}{l}717 \\
717 \\
717 \\
716 \\
707\end{array}$ & $\begin{array}{l}726 \\
726 \\
724 \\
723 \\
722\end{array}$ & $\begin{array}{l}--- \\
705 \\
705 \\
703 \\
704\end{array}$ & $\begin{array}{l}711 \\
708 \\
708 \\
709 \\
711\end{array}$ & $\begin{array}{l}703 \\
704 \\
703 \\
703 \\
701\end{array}$ & $\begin{array}{l}727 \\
728 \\
729 \\
728 \\
726\end{array}$ \\
\hline $\begin{array}{r}6 \\
7 \\
8 \\
9 \\
10\end{array}$ & $\begin{array}{l}--- \\
--- \\
--- \\
--\end{array}$ & $\begin{array}{l}-- \\
-- \\
=- \\
-- \\
--\end{array}$ & $\begin{array}{l}--- \\
--- \\
--- \\
---\end{array}$ & $\begin{array}{l}-\cdots \\
-- \\
-- \\
--\end{array}$ & $\begin{array}{l}--- \\
--- \\
--- \\
--\end{array}$ & $\begin{array}{l}--- \\
--- \\
--- \\
---\end{array}$ & $\begin{array}{l}706 \\
712 \\
716 \\
718 \\
720\end{array}$ & $\begin{array}{l}723 \\
723 \\
724 \\
724 \\
725\end{array}$ & $\begin{array}{l}699 \\
690 \\
691 \\
690 \\
696\end{array}$ & $\begin{array}{l}713 \\
715 \\
714 \\
716 \\
717\end{array}$ & $\begin{array}{l}704 \\
704 \\
705 \\
705 \\
708\end{array}$ & $\begin{array}{l}726 \\
726 \\
726 \\
--- \\
---\end{array}$ \\
\hline $\begin{array}{l}11 \\
12 \\
13 \\
14 \\
15\end{array}$ & $\begin{array}{l}--- \\
--- \\
--- \\
---\end{array}$ & $\begin{array}{l}--- \\
--- \\
--- \\
--- \\
---\end{array}$ & $\begin{array}{l}--- \\
--- \\
--- \\
--- \\
---\end{array}$ & $\begin{array}{l}--- \\
--- \\
--- \\
---\end{array}$ & $\begin{array}{l}--- \\
--- \\
--- \\
---\end{array}$ & $\begin{array}{l}--- \\
--- \\
--- \\
--- \\
--\end{array}$ & \begin{tabular}{l}
721 \\
\hdashline-- \\
723 \\
724 \\
724
\end{tabular} & $\begin{array}{l}726 \\
--- \\
715 \\
716\end{array}$ & $\begin{array}{l}699 \\
700 \\
700 \\
701 \\
702\end{array}$ & $\begin{array}{l}719 \\
719 \\
717 \\
712 \\
709\end{array}$ & $\begin{array}{l}709 \\
--- \\
--- \\
---\end{array}$ & $\begin{array}{l}--- \\
--- \\
722 \\
722 \\
727\end{array}$ \\
\hline $\begin{array}{l}16 \\
17 \\
18 \\
19 \\
20\end{array}$ & $\begin{array}{l}--- \\
--- \\
--- \\
---\end{array}$ & $\begin{array}{l}--- \\
--- \\
--- \\
---\end{array}$ & $\begin{array}{l}--- \\
--- \\
--- \\
--- \\
---\end{array}$ & $\begin{array}{l}--- \\
--- \\
--- \\
--\end{array}$ & $\begin{array}{l}--- \\
--- \\
--- \\
--- \\
--\end{array}$ & $\begin{array}{l}--- \\
--- \\
--- \\
--- \\
---\end{array}$ & $\begin{array}{l}725 \\
726 \\
726 \\
--- \\
726\end{array}$ & $\begin{array}{l}718 \\
722 \\
722 \\
721 \\
722\end{array}$ & $\begin{array}{l}701 \\
700 \\
700 \\
701 \\
701\end{array}$ & $\begin{array}{l}707 \\
704 \\
703 \\
702 \\
703\end{array}$ & $\begin{array}{l}--- \\
--- \\
708 \\
708\end{array}$ & $\begin{array}{l}731 \\
732 \\
734 \\
732 \\
728\end{array}$ \\
\hline $\begin{array}{l}21 \\
22 \\
23 \\
24 \\
25\end{array}$ & $\begin{array}{l}--- \\
--- \\
--- \\
--- \\
---\end{array}$ & $\begin{array}{l}-- \\
-- \\
-- \\
-- \\
--\end{array}$ & $\begin{array}{l}-- \\
-- \\
-- \\
--- \\
---\end{array}$ & $\begin{array}{l}-- \\
-- \\
-- \\
-- \\
--\end{array}$ & $\begin{array}{l}--- \\
--- \\
--- \\
--- \\
---\end{array}$ & $\begin{array}{l}--- \\
\cdots- \\
--- \\
---\end{array}$ & $\begin{array}{l}727 \\
727 \\
728 \\
728 \\
727\end{array}$ & $\begin{array}{l}723 \\
724 \\
720 \\
720 \\
722\end{array}$ & $\begin{array}{l}700 \\
703 \\
700 \\
699 \\
698\end{array}$ & $\begin{array}{l}705 \\
706 \\
704 \\
703 \\
704\end{array}$ & $\begin{array}{l}708 \\
708 \\
709 \\
710 \\
717\end{array}$ & $\begin{array}{l}735 \\
729 \\
730 \\
735 \\
726\end{array}$ \\
\hline $\begin{array}{l}26 \\
27 \\
28 \\
29 \\
30 \\
31\end{array}$ & $\begin{array}{l}--- \\
--- \\
--- \\
--- \\
--\end{array}$ & $\begin{array}{l}--- \\
--- \\
--- \\
--- \\
---\end{array}$ & $\begin{array}{l}--- \\
--- \\
--- \\
--- \\
-- \\
---\end{array}$ & $\begin{array}{l}--- \\
--- \\
--- \\
--- \\
--\end{array}$ & $\begin{array}{l}-- \\
-- \\
--\end{array}$ & $\begin{array}{l}718 \\
712 \\
712 \\
713 \\
717 \\
717\end{array}$ & $\begin{array}{l}727 \\
727 \\
727 \\
727 \\
726\end{array}$ & $\begin{array}{l}722 \\
722 \\
722 \\
724 \\
724 \\
--\end{array}$ & $\begin{array}{l}698 \\
701 \\
711 \\
730 \\
717\end{array}$ & $\begin{array}{l}705 \\
707 \\
710 \\
708 \\
707 \\
705\end{array}$ & $\begin{array}{l}722 \\
721 \\
722 \\
724 \\
726 \\
725\end{array}$ & $\begin{array}{l}717 \\
715 \\
717 \\
721 \\
718\end{array}$ \\
\hline
\end{tabular}

Daily mean water temperature, in degrees Celsius

\begin{tabular}{|c|c|c|c|c|c|c|c|c|c|c|c|}
\hline $\begin{array}{l}--- \\
--- \\
--- \\
--- \\
---\end{array}$ & $\begin{array}{l}-- \\
--- \\
--- \\
---\end{array}$ & $\begin{array}{l}--- \\
--- \\
--- \\
--- \\
---\end{array}$ & $\begin{array}{l}--- \\
--- \\
--- \\
--- \\
---\end{array}$ & $\begin{array}{l}--- \\
--- \\
-- \\
---\end{array}$ & $\begin{array}{l}--- \\
--- \\
--- \\
---\end{array}$ & $\begin{array}{l}9.3 \\
9.3 \\
9.3 \\
9.3 \\
9.5\end{array}$ & $\begin{array}{l}9.6 \\
9.6 \\
9.7 \\
9.7 \\
9.7\end{array}$ & $\begin{array}{r}--- \\
10.3 \\
10.3 \\
10.3 \\
10.4\end{array}$ & $\begin{array}{l}10.6 \\
10.6 \\
10.6 \\
10.6 \\
10.6\end{array}$ & $\begin{array}{l}10.4 \\
10.4 \\
10.4 \\
10.4 \\
10.4\end{array}$ & $\begin{array}{l}10.4 \\
10.4 \\
10.4 \\
10.4 \\
10.4\end{array}$ \\
\hline $\begin{array}{l}--- \\
--- \\
--- \\
--- \\
---\end{array}$ & $\begin{array}{l}--- \\
-- \\
-- \\
--\end{array}$ & $\begin{array}{l}--- \\
--- \\
--- \\
--- \\
---\end{array}$ & $\begin{array}{l}--- \\
--- \\
--- \\
---\end{array}$ & $\begin{array}{l}--- \\
--- \\
--- \\
---\end{array}$ & $\begin{array}{l}--- \\
--- \\
--- \\
---\end{array}$ & $\begin{array}{l}9.4 \\
9.4 \\
9.5 \\
9.5 \\
9.5\end{array}$ & $\begin{array}{l}9.8 \\
9.8 \\
9.8 \\
9.9 \\
9.9\end{array}$ & $\begin{array}{l}10.4 \\
10.4 \\
10.4 \\
10.4 \\
10.4\end{array}$ & $\begin{array}{l}10.6 \\
10.6 \\
10.6 \\
10.6 \\
10.6\end{array}$ & $\begin{array}{l}10.4 \\
10.4 \\
10.4 \\
10.4 \\
10.4\end{array}$ & $\begin{array}{l}10.4 \\
10.4 \\
10.4 \\
10.4 \\
10.3\end{array}$ \\
\hline $\begin{array}{l}--- \\
--- \\
--- \\
--- \\
---\end{array}$ & $\begin{array}{l}--- \\
--- \\
-- \\
--\end{array}$ & $\begin{array}{l}--- \\
--- \\
--- \\
--- \\
---\end{array}$ & $\begin{array}{l}--- \\
--- \\
--- \\
--- \\
---\end{array}$ & $\begin{array}{l}--- \\
--- \\
--- \\
---\end{array}$ & $\begin{array}{l}--- \\
--- \\
--- \\
---\end{array}$ & $\begin{array}{l}9.5 \\
9.6 \\
9.6 \\
9.6\end{array}$ & $\begin{array}{r}9.9 \\
9.9 \\
10.0\end{array}$ & $\begin{array}{l}10.4 \\
10.4 \\
10.4 \\
10.4 \\
10.5\end{array}$ & $\begin{array}{l}10.6 \\
10.5 \\
10.5 \\
10.5 \\
10.5\end{array}$ & $\begin{array}{r}10.4 \\
- \\
- \\
- \\
-\end{array}$ & $\begin{array}{l}10.4 \\
10.4 \\
10.4 \\
10.4 \\
10.4\end{array}$ \\
\hline $\begin{array}{l}--- \\
--- \\
--- \\
--- \\
---\end{array}$ & $\begin{array}{l}--- \\
--- \\
--- \\
---\end{array}$ & $\begin{array}{l}--- \\
--- \\
--- \\
--- \\
---\end{array}$ & $\begin{array}{l}--- \\
--- \\
--- \\
--- \\
---\end{array}$ & $\begin{array}{l}--- \\
--- \\
--- \\
---\end{array}$ & $\begin{array}{l}--- \\
--- \\
--- \\
--- \\
---\end{array}$ & $\begin{array}{l}9.6 \\
9.6 \\
9.6 \\
9.6\end{array}$ & $\begin{array}{l}10.0 \\
10.1 \\
10.1 \\
10.1 \\
10.1\end{array}$ & $\begin{array}{l}10.5 \\
10.5 \\
10.5 \\
10.6 \\
10.6\end{array}$ & $\begin{array}{l}10.5 \\
10.5 \\
10.5 \\
10.5 \\
10.5\end{array}$ & $\begin{array}{c}-.- \\
10.5 \\
10.4\end{array}$ & $\begin{array}{l}10.3 \\
10.3 \\
10.3 \\
10.3 \\
10.3\end{array}$ \\
\hline $\begin{array}{l}--- \\
--- \\
--- \\
--- \\
---\end{array}$ & $\begin{array}{l}--- \\
--- \\
--- \\
--- \\
---\end{array}$ & $\begin{array}{l}--- \\
--- \\
--- \\
--- \\
---\end{array}$ & $\begin{array}{l}--- \\
--- \\
--- \\
--- \\
---\end{array}$ & $\begin{array}{l}--- \\
-- \\
-- \\
--\end{array}$ & $\begin{array}{l}--- \\
--- \\
--- \\
---\end{array}$ & $\begin{array}{l}9.6 \\
9.6 \\
9.6 \\
9.6 \\
9.6\end{array}$ & $\begin{array}{l}10.1 \\
10.1 \\
10.2 \\
10.2 \\
10.2\end{array}$ & $\begin{array}{l}10.6 \\
10.6 \\
10.5 \\
10.6 \\
10.6\end{array}$ & $\begin{array}{l}10.5 \\
10.5 \\
10.5 \\
10.5 \\
10.5\end{array}$ & $\begin{array}{l}10.4 \\
10.4 \\
10.5 \\
10.5 \\
10.5\end{array}$ & $\begin{array}{l}10.3 \\
10.3 \\
10.3 \\
10.3 \\
10.3\end{array}$ \\
\hline $\begin{array}{l}--- \\
--- \\
--- \\
--- \\
--- \\
---\end{array}$ & $\begin{array}{l}-- \\
-- \\
-- \\
-- \\
--\end{array}$ & $\begin{array}{l}--- \\
--- \\
--- \\
--- \\
--- \\
---\end{array}$ & $\begin{array}{l}--- \\
--- \\
--- \\
--- \\
--- \\
--\end{array}$ & $\begin{array}{l}--- \\
--- \\
---\end{array}$ & $\begin{array}{l}9.1 \\
9.2 \\
9.2 \\
9.2 \\
9.2 \\
9.3\end{array}$ & $\begin{array}{l}9.5 \\
9.5 \\
9.6 \\
9.6 \\
9.6\end{array}$ & $\begin{array}{r}10.2 \\
10.3 \\
10.3 \\
10.3 \\
10.3 \\
\end{array}$ & $\begin{array}{l}10.6 \\
10.6 \\
10.6 \\
10.6 \\
10.6\end{array}$ & $\begin{array}{l}10.6 \\
10.5 \\
10.5 \\
10.4 \\
10.4 \\
10.4\end{array}$ & $\begin{array}{l}10.5 \\
10.5 \\
10.5 \\
10.5 \\
10.4 \\
10.4\end{array}$ & $\begin{array}{l}10.3 \\
10.3 \\
10.3 \\
10.3 \\
10.3\end{array}$ \\
\hline
\end{tabular}


Table $18 .--$ Daily median $\mathrm{pH}$ at Big Spring

[Data before May 4 from a corresponding spring pool 8 age height of

approximately 8.5 feet, and after May 4 from approximately 10 feet;

---, data not available to calculate median values]

\begin{tabular}{|c|c|c|c|c|c|c|c|c|c|c|c|c|}
\hline Day & Oct & Nov & Dec & $\operatorname{Jan}$ & Feb & Mar & Apr & May & June & July & Aus & sept \\
\hline \multicolumn{13}{|c|}{ Daily median ph, in units } \\
\hline $\begin{array}{l}1 \\
2 \\
3 \\
4 \\
5\end{array}$ & $\begin{array}{l}--- \\
--- \\
--- \\
---\end{array}$ & $\begin{array}{l}--- \\
--- \\
--- \\
---\end{array}$ & $\begin{array}{l}--- \\
--- \\
--- \\
---\end{array}$ & $\begin{array}{l}--- \\
--- \\
--- \\
--- \\
---\end{array}$ & $\begin{array}{l}--- \\
--- \\
--- \\
--\end{array}$ & $\begin{array}{l}--- \\
--- \\
--- \\
---\end{array}$ & $\begin{array}{r}7.41 \\
7.42 \\
7.42 \\
7.42 \\
-\end{array}$ & $\begin{array}{l}7.61 \\
7.60 \\
7.58 \\
7.14 \\
7.10\end{array}$ & $\begin{array}{l}--- \\
6.76 \\
6.77 \\
6.82 \\
6.87\end{array}$ & $\begin{array}{l}6.92 \\
6.92 \\
6.91 \\
6.90 \\
6.88\end{array}$ & $\begin{array}{l}6.75 \\
6.74 \\
6.74 \\
6.75 \\
6.75\end{array}$ & $\begin{array}{l}6.75 \\
6.75 \\
6.76 \\
6.76 \\
6.77\end{array}$ \\
\hline $\begin{array}{r}6 \\
7 \\
8 \\
9 \\
10\end{array}$ & $\begin{array}{l}--- \\
--- \\
-- \\
--\end{array}$ & $\begin{array}{l}-- \\
--- \\
-- \\
--\end{array}$ & $\begin{array}{l}-- \\
--- \\
--- \\
---\end{array}$ & $\begin{array}{l}--1 \\
--1 \\
--- \\
---\end{array}$ & $\begin{array}{l}--- \\
--- \\
-- \\
--\end{array}$ & $\begin{array}{l}-- \\
=- \\
-- \\
-- \\
--\end{array}$ & $\begin{array}{l}--- \\
--- \\
--- \\
---\end{array}$ & $\begin{array}{l}7.09 \\
7.08 \\
7.07 \\
7.07 \\
7.05\end{array}$ & $\begin{array}{l}6.91 \\
6.83 \\
6.87 \\
6.92 \\
6.99\end{array}$ & $\begin{array}{l}6.88 \\
6.86 \\
6.65 \\
6.67 \\
6.75\end{array}$ & $\begin{array}{l}6.75 \\
6.74 \\
6.75 \\
6.75 \\
6.75\end{array}$ & $\begin{array}{l}6.77 \\
6.77 \\
6.76 \\
6.77 \\
6.76\end{array}$ \\
\hline $\begin{array}{l}11 \\
12 \\
13 \\
14 \\
15\end{array}$ & $\begin{array}{l}--- \\
-- \\
-- \\
--\end{array}$ & $\begin{array}{l}--- \\
--- \\
--- \\
--\end{array}$ & $\begin{array}{l}--- \\
--- \\
--- \\
--\end{array}$ & $\begin{array}{l}=- \\
--- \\
--- \\
--\end{array}$ & $\begin{array}{l}--- \\
-- \\
--- \\
--\end{array}$ & $\begin{array}{l}--- \\
=- \\
--- \\
---\end{array}$ & $\begin{array}{l}--0 \\
=-1 \\
--2 \\
7.47\end{array}$ & $\begin{array}{r}7.04 \\
- \\
6.99 \\
6.98\end{array}$ & $\begin{array}{l}7.02 \\
7.01 \\
7.00 \\
6.99 \\
6.97\end{array}$ & $\begin{array}{l}6.82 \\
6.94 \\
6.92 \\
6.85 \\
6.80\end{array}$ & $\begin{array}{c}6.75 \\
=- \\
- \\
-\end{array}$ & $\begin{array}{l}6.76 \\
6.77 \\
6.78 \\
6.76 \\
6.77\end{array}$ \\
\hline $\begin{array}{l}16 \\
17 \\
18 \\
19 \\
20\end{array}$ & $\begin{array}{l}--- \\
-- \\
--- \\
--\end{array}$ & $\begin{array}{l}--- \\
--- \\
--- \\
--\end{array}$ & $\begin{array}{l}--- \\
--- \\
--- \\
--\end{array}$ & $\begin{array}{l}-- \\
-- \\
-- \\
--\end{array}$ & $\begin{array}{l}--- \\
--- \\
--- \\
--\end{array}$ & $\begin{array}{l}--- \\
--- \\
--- \\
---\end{array}$ & $\begin{array}{r}7.48 \\
7.47 \\
7.50 \\
-2 \\
7.52\end{array}$ & $\begin{array}{l}7.00 \\
6.99 \\
6.99 \\
6.98 \\
6.95\end{array}$ & $\begin{array}{l}6.97 \\
6.97 \\
6.96 \\
6.96 \\
6.95\end{array}$ & $\begin{array}{l}6.80 \\
6.80 \\
6.81 \\
6.81 \\
6.79\end{array}$ & $\begin{array}{r}--1 \\
--- \\
6.81 \\
6.81\end{array}$ & $\begin{array}{l}6.76 \\
6.74 \\
6.74 \\
6.75 \\
6.77\end{array}$ \\
\hline $\begin{array}{l}21 \\
22 \\
23 \\
24 \\
25\end{array}$ & $\begin{array}{l}--- \\
--- \\
--- \\
---\end{array}$ & $\begin{array}{l}--- \\
--- \\
--- \\
--\end{array}$ & $\begin{array}{l}--- \\
--- \\
--- \\
--- \\
---\end{array}$ & $\begin{array}{l}--- \\
--- \\
--- \\
---\end{array}$ & $\begin{array}{l}-- \\
-- \\
-- \\
-- \\
--\end{array}$ & 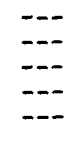 & $\begin{array}{l}7.55 \\
7.56 \\
7.57 \\
7.59 \\
7.59\end{array}$ & $\begin{array}{l}6.96 \\
6.97 \\
6.96 \\
6.94 \\
6.93\end{array}$ & $\begin{array}{l}6.95 \\
6.94 \\
6.95 \\
6.95 \\
6.94\end{array}$ & $\begin{array}{l}6.78 \\
6.77 \\
6.77 \\
6.77 \\
6.77\end{array}$ & $\begin{array}{l}6.81 \\
6.81 \\
6.82 \\
6.81 \\
6.82\end{array}$ & $\begin{array}{l}6.75 \\
6.76 \\
6.87 \\
6.86 \\
6.85\end{array}$ \\
\hline $\begin{array}{l}26 \\
27 \\
28 \\
29 \\
30 \\
31\end{array}$ & $\begin{array}{l}--- \\
--- \\
--- \\
--- \\
---\end{array}$ & $\begin{array}{l}--- \\
--- \\
--- \\
---\end{array}$ & $\begin{array}{l}-- \\
-- \\
-- \\
-- \\
--\end{array}$ & $\begin{array}{l}--- \\
--\overline{-} \\
--- \\
--- \\
---\end{array}$ & $\overline{-}$ & $\begin{array}{l}7.38 \\
7.39 \\
7.38 \\
7.39 \\
7.40 \\
7.41\end{array}$ & $\begin{array}{l}7.60 \\
7.61 \\
7.60 \\
7.61 \\
7.61\end{array}$ & $\begin{array}{l}6.94 \\
6.95 \\
6.96 \\
6.96 \\
6.96 \\
-\end{array}$ & $\begin{array}{l}6.94 \\
6.92 \\
6.91 \\
6.92 \\
6.91\end{array}$ & $\begin{array}{l}6.76 \\
6.75 \\
6.75 \\
6.74 \\
6.75 \\
6.74\end{array}$ & $\begin{array}{l}6.81 \\
6.79 \\
6.79 \\
6.77 \\
6.77 \\
6.75\end{array}$ & $\begin{array}{l}6.84 \\
6.85 \\
6.86 \\
6.82 \\
6.83\end{array}$ \\
\hline
\end{tabular}



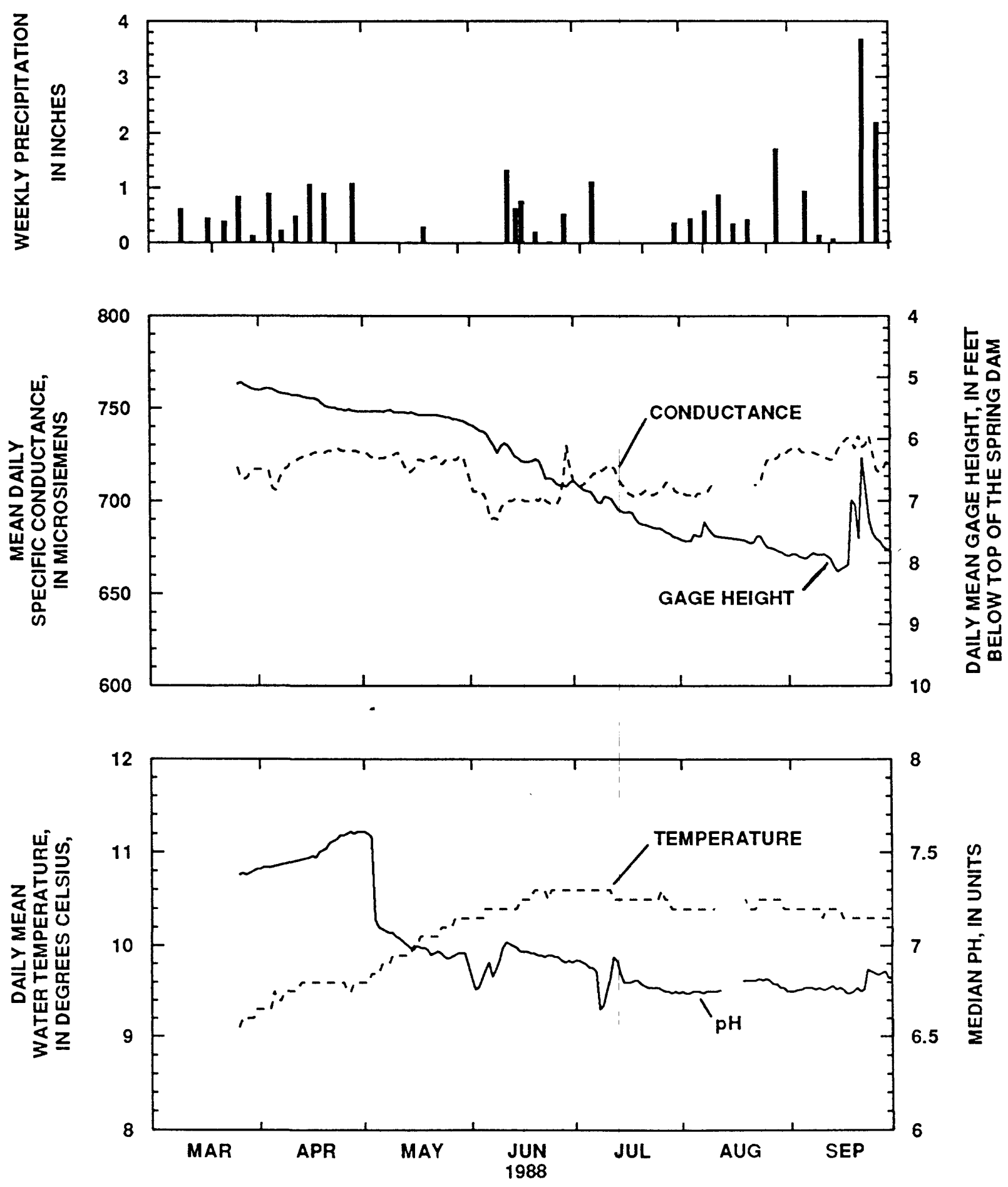

Figure 6.--Weekly precipitation, daily mean specific conductance, gage height, water temperature, and daily median $\mathrm{pH}$ at Big Spring, Clayton County, Iowa, March-September, 1988. 


\section{Seepage study}

Stream discharge was measured at sites(fig. 1) in the study area after an extended period without rain (June 28-29) to determine areas where streamflow was being lost to the ground-water system through seepage and to determine areas where water was flowing into the streams from shallow aquifers. Measurements were also made to quantify the gain or loss to the stream. Water samples were collected concurrently to document nutrient inflow from ground water and outflow into the underlying aquifer. The results of the study are presented in table 19 and figures 7 and 8 . Discussion and interpretation of the results is beyond the scope of this report and will be presented in future reports. The results of discharge measurements and chemical analyses of samples from sites on Roberts Creek and a stretch of Silver Creek are summarized here.

\section{Discharge}

Discharge increased in Roberts Creek from $0.10 \mathrm{ft}^{3} / \mathrm{s}$ at site $\mathrm{RC13}$, the most upstream site, to $5.17 \mathrm{ft}^{3} / \mathrm{s}$ at $\mathrm{RC18}$ approximately 17 miles downstream (fig. 7). Streamflow in this stretch partially originated from three tributaries, West Branch Roberts Creek, Deer Creek, and Silver Creek. West Branch Roberts Creek added $0.82 \mathrm{ft}^{3} / \mathrm{s}$, Deer Creek added approximately 0.77 $\mathrm{ft}^{3} / \mathrm{s}$ and Silver Creek added approximately $0.36 \mathrm{ft}^{3} / \mathrm{s}$. Discharge decreased in a 9.5 mile stretch downstream of site $\mathrm{RCl} 8 \mathrm{from} 5.17 \mathrm{ft}^{3} / \mathrm{s}$ to $2.11 \mathrm{ft}^{3} / \mathrm{s}$ at site RC2. Total discharge lost from Roberts Creek during the seepage study was $3.25 \mathrm{ft}^{3} / \mathrm{s}$. This includes $0.33 \mathrm{ft}^{3} / \mathrm{s}$ lost from sites $\mathrm{RC1} 6$ to $\mathrm{RC18}$ and $3.06 \mathrm{ft}^{3} / \mathrm{s}$ lost from sites $\mathrm{RC} 18$ to $\mathrm{RC} 2$. During subsequent measurements (table 20), Roberts Creek lost from approximately 1.5 to $3.1 \mathrm{ft}^{3} / \mathrm{s}$ from site RC18 to RC2. The losses are from 31 to 99 percent of the discharge measured at site RC18.

Determination of seepage in Silver Creek is complicated by non-uniform point-source discharges in the headwaters of the stream. A municipality discharges upstream of site SC10 and an industrial user discharges waste water upstream of site SC13. Discharges for sites SC10 on East Fork Silver Creek to $\mathrm{SCl}$ on Silver Creek are shown in figure 8. Discharges were 0.12 and 1.19 $\mathrm{ft}^{3} / \mathrm{s}$ at sites $\mathrm{SClO}$ and SC13, respectively. The combined flow of East Fork and the main stem of Silver Creek (SC6 plus SC5) was $2.12 \mathrm{ft}^{3} / \mathrm{s}$. Downstream at site $\mathrm{SCl}$ discharge had decreased to $0.81 \mathrm{ft}^{3} / \mathrm{s}$. A measurement also was made at site SC14, on a tributary of Silver Creek, however, this water did not reach the main stem. The total loss of discharge in silver Creek was approximately $1.46 \mathrm{ft}^{3} / \mathrm{s}$. The discharge loss downstream of site $\mathrm{SCl}$ on Silver Creek was not used in calculating loss from the stream because it is not known whether discharge is representative of the intermittent point source discharge or of natural flow. 
Table 19. - Field measurements and chemical analyses of samples from streams in the Big Spring Basin during low-flow conditions [Dissolved chemical constituents in milligrams per liter]

[ - missing data; $\mathrm{ft}^{3} / \mathrm{s}$, cubic feet per second: $\mu \mathrm{s} / \mathrm{cm}$, microsiemens per centimeter; C, degrees celsius; N, nitrogen; $P$, phosphorus; C, carbon; <, less than]

\begin{tabular}{|c|c|c|c|c|c|c|c|c|c|c|c|c|}
\hline $\begin{array}{l}\text { Station } \\
\text { number }\end{array}$ & Date & Time & $\begin{array}{l}\text { Stream- } \\
\text { flgh }^{-} \\
\left.\text {(ft }^{3} / \mathrm{s}\right)\end{array}$ & $\begin{array}{l}\text { Spe- } \\
\text { cific } \\
\text { con- } \\
\text { duct- } \\
\text { ance } \\
(\mu S / \mathrm{cm})\end{array}$ & $\underset{\text { (units) }}{\mathrm{pH}}$ & $\begin{array}{l}\text { Temper- } \\
\text { ature } \\
\text { ( C) }\end{array}$ & $\begin{array}{l}\text { Chlo- } \\
\text { ride }\end{array}$ & $\begin{array}{l}\text { Nitrite } \\
\text { plus } \\
\text { nitrate } \\
\text { (as N) }\end{array}$ & $\begin{array}{c}\text { Ammonia } \\
\text { (as N) }\end{array}$ & $\begin{array}{l}\text { Organic } \\
\text { nitrogen } \\
\text { (as N) }\end{array}$ & $\begin{array}{l}\text { Ortho- } \\
\text { phos- } \\
\text { phorus } \\
\text { (as P) }\end{array}$ & $\begin{array}{l}\text { Total } \\
\text { organic } \\
\text { carbon } \\
\text { (as C) }\end{array}$ \\
\hline $\begin{array}{l}\text { HS5 } \\
\text { HS6 } \\
\text { HS4 } \\
\text { HS2 } \\
\text { HS3 }\end{array}$ & $\begin{array}{l}6-29-88 \\
6-29-88 \\
6-29-88 \\
6-29-88 \\
6-29-88\end{array}$ & $\begin{array}{l}1345 \\
1500 \\
1600 \\
1700 \\
1730\end{array}$ & $\begin{array}{r}0.20 \\
.29 \\
.36 \\
.27 \\
.71\end{array}$ & $\begin{array}{l}750 \\
700 \\
695 \\
690 \\
625\end{array}$ & $\begin{array}{l}8.0 \\
8.1 \\
7.9 \\
-. . \\
8.3\end{array}$ & $\begin{array}{l}17.0 \\
17.0 \\
15.0 \\
16.5 \\
18.0\end{array}$ & $\begin{array}{l}29 \\
19 \\
17 \\
18 \\
18\end{array}$ & $\begin{array}{l}0.2 \\
5.7 \\
9.0 \\
8.0 \\
5.7\end{array}$ & $\begin{array}{l}<0.1 \\
<.1 \\
<.1 \\
<.3\end{array}$ & $\begin{array}{r}0.7 \\
3.3 \\
.6 \\
.6 \\
.4\end{array}$ & $\begin{array}{r}0.3 \\
.2 \\
<.1 \\
.3 \\
.1\end{array}$ & $\begin{array}{l}2.4 \\
3.3 \\
1.8 \\
7.5 \\
2.6\end{array}$ \\
\hline $\begin{array}{l}\text { RS1 } \\
\text { RC13 } \\
\text { RC21 } \\
\text { RC20 } \\
\text { RC11 }\end{array}$ & $\begin{array}{l}6-29-88 \\
6-29-88 \\
6-29-88 \\
6-29-88 \\
6-29-88\end{array}$ & $\begin{array}{l}1700 \\
1530 \\
1430 \\
1345 \\
1145\end{array}$ & $\begin{array}{r}.30 \\
.10 \\
.82 \\
2.54 \\
2.79\end{array}$ & $\begin{array}{l}600 \\
680 \\
619 \\
621 \\
640\end{array}$ & $\begin{array}{l}8.3 \\
8.3 \\
8.5 \\
8.4 \\
8.7\end{array}$ & $\begin{array}{l}20.0 \\
18.0 \\
18.5 \\
19.0 \\
18.5\end{array}$ & $\begin{array}{l}16 \\
23 \\
22 \\
18 \\
18\end{array}$ & $\begin{array}{l}4.7 \\
4.3 \\
7.3 \\
4.9 \\
4.7\end{array}$ & $\begin{array}{r}<.1 \\
.3 \\
.1 \\
.1 \\
<.1\end{array}$ & $\begin{array}{r}.8 \\
1.0 \\
.3 \\
.3 \\
.5\end{array}$ & $\begin{array}{r}.2 \\
.7 \\
<.1 \\
.3 \\
.2\end{array}$ & $\begin{array}{l}3.7 \\
4.5 \\
2.2 \\
2.8 \\
2.8\end{array}$ \\
\hline $\begin{array}{l}\mathrm{RC} 10 \\
\mathrm{RC} 15 \\
\mathrm{DC} 4 \\
\mathrm{DC} 2 \\
\mathrm{~F} 45\end{array}$ & $\begin{array}{l}6-29-88 \\
6-28-88 \\
6-28-88 \\
6-28-88 \\
6-28-88\end{array}$ & $\begin{array}{l}0915 \\
1215 \\
1845 \\
1345 \\
1530\end{array}$ & $\begin{array}{r}3.59 \\
3.44 \\
.42 \\
.77 \\
4.37\end{array}$ & $\begin{array}{l}630 \\
615 \\
561 \\
680 \\
598\end{array}$ & $\begin{array}{l}8.2 \\
8.1 \\
8.4 \\
8.3 \\
8.6\end{array}$ & $\begin{array}{l}19.0 \\
22.5 \\
26.5 \\
22.0 \\
22.0\end{array}$ & $\begin{array}{l}21 \\
20 \\
15 \\
16 \\
20\end{array}$ & $\begin{array}{l}3.4 \\
1.5 \\
4.4 \\
5.4 \\
2.2\end{array}$ & 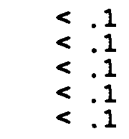 & $\begin{array}{r}.3 \\
1.0 \\
.5 \\
1.6 \\
.6\end{array}$ & $\begin{array}{r}.1 \\
.2 \\
.1 \\
.1 \\
.1\end{array}$ & $\begin{array}{r}3.7 \\
11 \\
2.4 \\
2.2 \\
4.1\end{array}$ \\
\hline $\begin{array}{l}\mathrm{RC} 16 \\
\mathrm{RC} 17 \\
\mathrm{SC} 10 \\
\mathrm{SC} 13 \\
\mathrm{SC} 3\end{array}$ & $\begin{array}{l}6-28-88 \\
6-28-88 \\
6-29-88 \\
6-29-88 \\
6-29-88\end{array}$ & $\begin{array}{l}1715 \\
1130 \\
0815 \\
0900 \\
1135\end{array}$ & $\begin{array}{r}4.86 \\
4.67 \\
.12 \\
1.19 \\
1.24\end{array}$ & $\begin{array}{r}574 \\
595 \\
1,280 \\
1,000 \\
1,135\end{array}$ & $\begin{array}{l}8.7 \\
-- \\
-- \\
-- \\
--\end{array}$ & $\begin{array}{l}28.5 \\
23.5 \\
12.0 \\
30.0 \\
22.0\end{array}$ & $\begin{array}{r}21 \\
22 \\
200 \\
64 \\
80\end{array}$ & $\begin{array}{r}1.2 \\
2.2 \\
27 \\
<.1 \\
1.4\end{array}$ & $\begin{array}{l}<.1 \\
<.1 \\
<.1 \\
3.1 \\
4.9\end{array}$ & $\begin{array}{r}.4 \\
.4 \\
1.2 \\
5.2 \\
2.0\end{array}$ & $\begin{array}{r}.1 \\
.3 \\
6.2 \\
3.8 \\
4.4\end{array}$ & $\begin{array}{l}4.1 \\
4.2 \\
6.1 \\
2.4 \\
1.6\end{array}$ \\
\hline $\begin{array}{l}\text { SC6 } \\
\text { SCU1 } \\
\text { SCU2 } \\
\text { L23 } \\
\text { SC5 }\end{array}$ & $\begin{array}{l}6-29-88 \\
6-29-88 \\
6-29-88 \\
6-29-88 \\
6-28-88\end{array}$ & $\begin{array}{l}1515 \\
1650 \\
1730 \\
1805 \\
1905\end{array}$ & $\begin{array}{r}1.43 \\
.12 \\
-. \\
.52 \\
.69\end{array}$ & $\begin{array}{r}1,090 \\
630 \\
710 \\
680 \\
665\end{array}$ & $\begin{array}{l}-- \\
-- \\
-- \\
--\end{array}$ & $\begin{array}{l}19.0 \\
26.5 \\
18.5 \\
27.5 \\
25.5\end{array}$ & $\begin{array}{l}90 \\
18 \\
20 \\
24 \\
23\end{array}$ & $\begin{array}{r}4.4 \\
15 \\
7.6 \\
8.2\end{array}$ & $\begin{array}{r}7.8 \\
. .2 \\
<.1 \\
<.8 \\
.1\end{array}$ & $\begin{array}{r}3.0 \\
1.9 \\
.3 \\
2.8 \\
.5\end{array}$ & $\begin{array}{r}4.3 \\
<.1 \\
<.1 \\
<.1\end{array}$ & $\begin{array}{l}1.7 \\
5.1 \\
1.3 \\
6.2 \\
2.8\end{array}$ \\
\hline $\begin{array}{l}\mathrm{SC} 1 \\
\mathrm{SC} 14 \\
\mathrm{SC2} \\
\mathrm{SC} 4 \\
\mathrm{RC} 18\end{array}$ & $\begin{array}{l}6-29-88 \\
6-29-88 \\
6-28-88 \\
6-28-88 \\
6-28-88\end{array}$ & $\begin{array}{l}1900 \\
1030 \\
1445 \\
1240 \\
1350\end{array}$ & $\begin{array}{r}.81 \\
.15 \\
.25 \\
.36 \\
5.17\end{array}$ & $\begin{array}{r}1,130 \\
710 \\
1,310 \\
1,490 \\
600\end{array}$ & $\begin{array}{l}-- \\
-- \\
-- \\
--\end{array}$ & $\begin{array}{l}19.5 \\
15.5 \\
29.0 \\
22.0 \\
24.0\end{array}$ & $\begin{array}{r}100 \\
34 \\
160 \\
250 \\
23\end{array}$ & $\begin{array}{r}.5 \\
1.7 \\
1.0 \\
3.0 \\
2.5\end{array}$ & $\begin{array}{r}9.5 \\
<\quad .1 \\
<.1 \\
1.2 \\
3.9\end{array}$ & $\begin{array}{r}.5 \\
.2 \\
.5 \\
.8 \\
1.2\end{array}$ & $\begin{array}{r}4.9 \\
.1 \\
.2 \\
2.2 \\
3.2\end{array}$ & $\begin{array}{l}6.5 \\
2.1 \\
4.2 \\
5.5 \\
8.7\end{array}$ \\
\hline $\begin{array}{l}F 47 \\
R C 19 \\
R C 19 \\
R C 24 \\
R C 23\end{array}$ & $\begin{array}{l}6-28-88 \\
6-28-88 \\
6-29-88 \\
6-29-88 \\
6-29-88\end{array}$ & $\begin{array}{l}1200 \\
1315 \\
0900 \\
1100\end{array}$ & $\begin{array}{l}4.02 \\
3.38 \\
4.24 \\
3.84 \\
3.49\end{array}$ & $\begin{array}{r}670 \\
630 \\
-- \\
630 \\
590\end{array}$ & $\begin{array}{l}8.3 \\
8.4 \\
-- \\
8.0 \\
8.4\end{array}$ & $\begin{array}{l}23.0 \\
24.0 \\
-- \\
21.0 \\
21.0\end{array}$ & $\begin{array}{l}24 \\
26 \\
-- \\
32 \\
24\end{array}$ & $\begin{array}{r}.7 \\
.8 \\
.5 \\
.2\end{array}$ & $\begin{array}{l}<.1 \\
<.1 \\
<.1 \\
<.1\end{array}$ & $\begin{array}{r}.7 \\
.9 \\
-1.1 \\
.5\end{array}$ & $\begin{array}{c}.3 \\
-3 \\
.3 \\
.3\end{array}$ & $\begin{array}{l}5.6 \\
7.1 \\
-. \\
5.0 \\
4.9\end{array}$ \\
\hline $\begin{array}{l}\mathrm{RC22} \\
\mathrm{RC2} \\
\mathrm{HC} 1 \\
\mathrm{HC2}\end{array}$ & $\begin{array}{l}6-28-88 \\
6-28-88 \\
6-28-88 \\
6-28-88\end{array}$ & $\begin{array}{l}1430 \\
1530 \\
1700 \\
1800\end{array}$ & $\begin{array}{r}2.63 \\
2.11 \\
.59 \\
.52\end{array}$ & $\begin{array}{l}580 \\
533 \\
700 \\
710\end{array}$ & $\begin{array}{l}8.9 \\
9.0 \\
8.4 \\
8.1\end{array}$ & $\begin{array}{l}27.0 \\
30.0 \\
24.0 \\
26.0\end{array}$ & $\begin{array}{l}24 \\
23 \\
18 \\
18\end{array}$ & $\begin{array}{r}<.1 \\
.2 \\
5.2 \\
4.4\end{array}$ & $\begin{array}{l}<.1 \\
<.1 \\
<.1 \\
<.1\end{array}$ & $\begin{array}{r}.6 \\
.7 \\
1.5 \\
.4\end{array}$ & $\begin{array}{r}.3 \\
<.2 \\
<.1 \\
<.1\end{array}$ & $\begin{array}{l}6.6 \\
6.4 \\
5.0 \\
2.3\end{array}$ \\
\hline
\end{tabular}



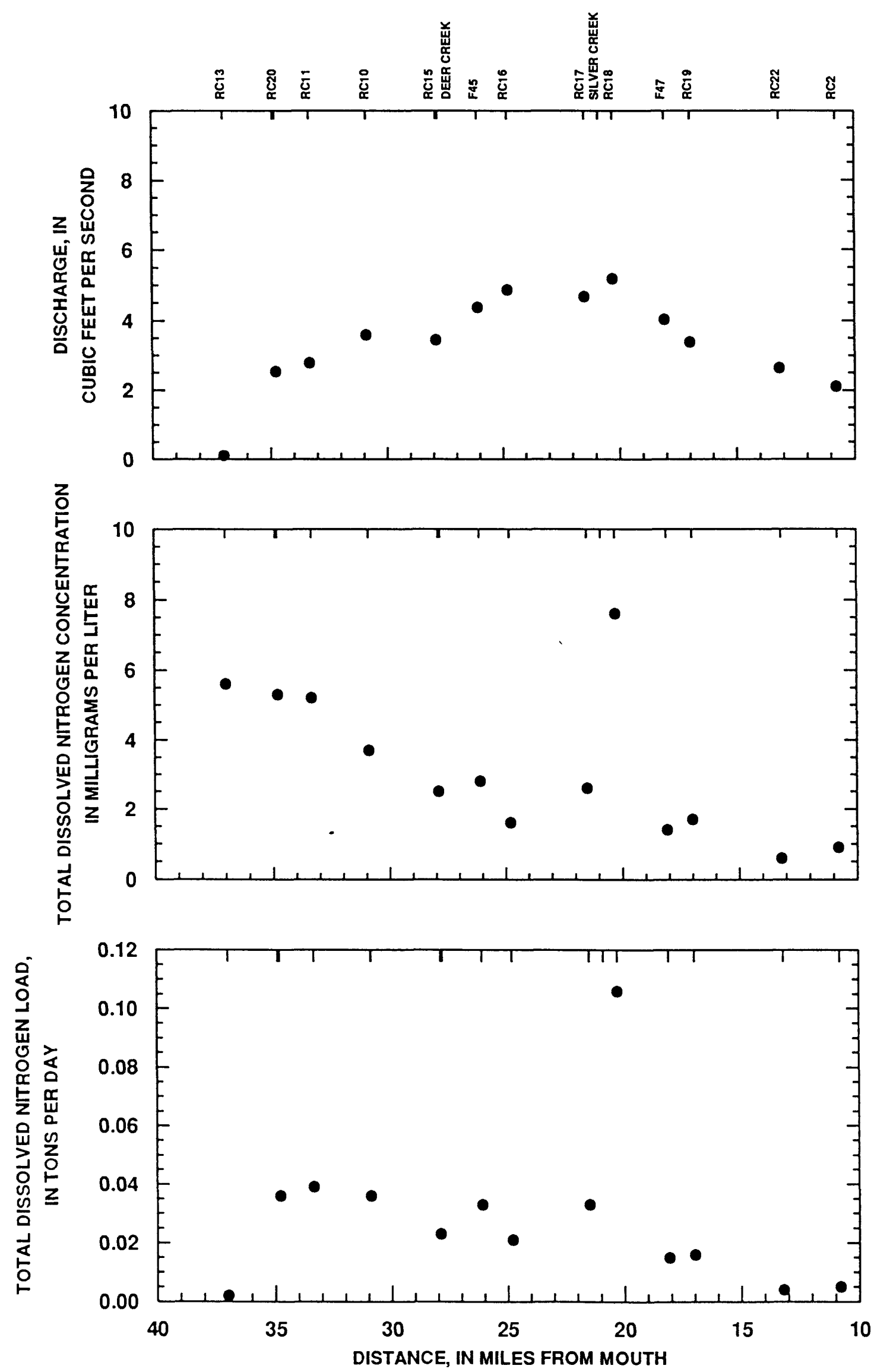

Figure 7.--Discharge, total dissolved nitrogen concentration, and total dissolved nitrogen load in Roberts Creek, June 28-29, 1988. 

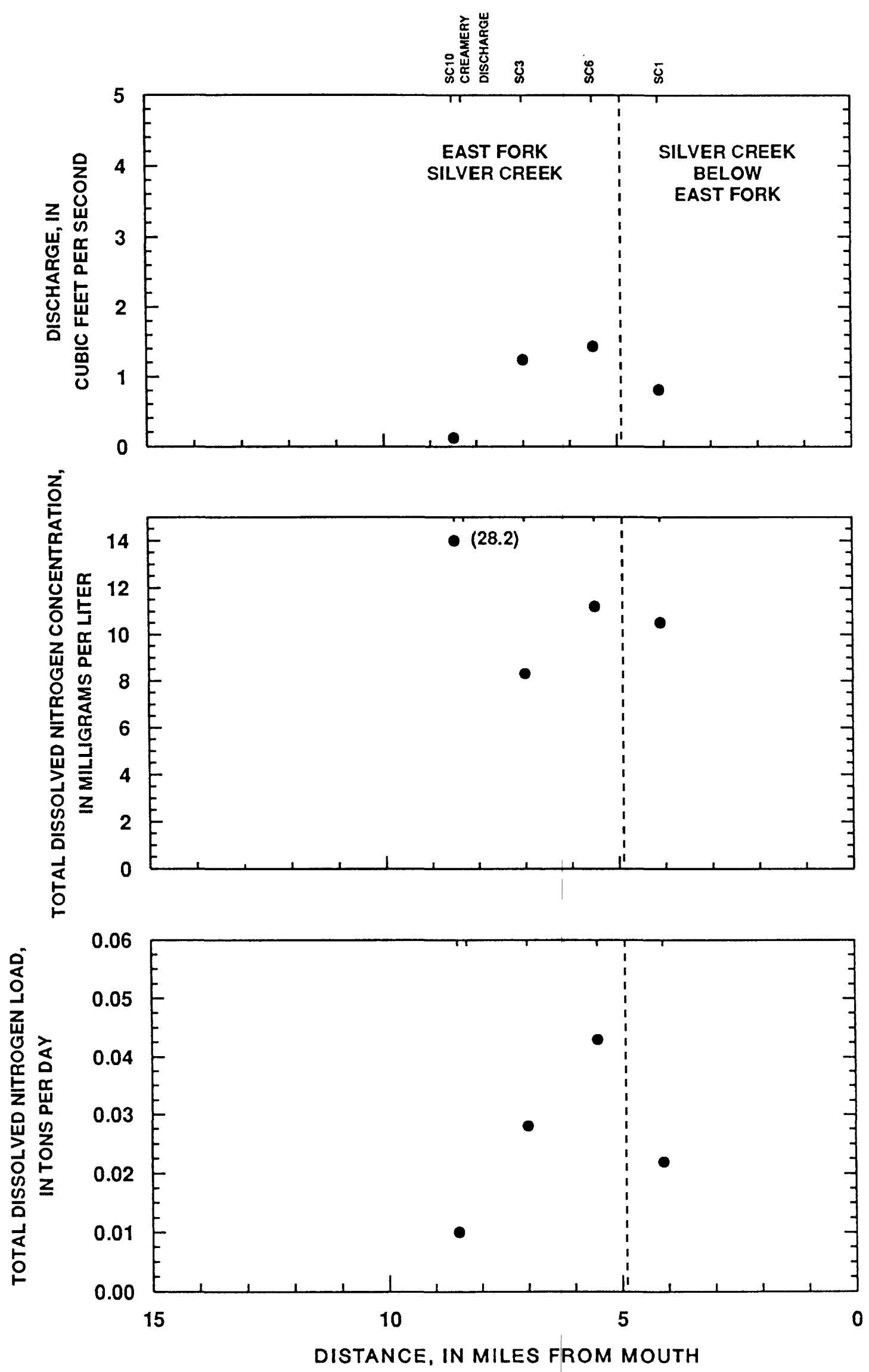

Figure 8.--Discharge, total dissolved nitrogen concentration, and total dissolved nitrogen load in East Fork Silver Creek and Silver Creek, June 28-29, 1988. 
Table 20.--Seepage in a selected reach of Roberts Creek [Discharge in cubic feet per second]

\begin{tabular}{lcccc}
\hline \multirow{2}{*}{ Date } & \multicolumn{2}{c}{ Discharge } & $\begin{array}{l}\text { Net } \\
\text { loss }\end{array}$ & $\begin{array}{l}\text { Percent } \\
\text { loss }\end{array}$ \\
\cline { 2 - 5 } & RC18 & RC2 & & 59 \\
\hline $6 / 28 / 88$ & 5.17 & 2.11 & 3.06 & 50 \\
$7 / 19 / 88$ & 4.39 & 1.32 & 3.07 & 70 \\
$8 / 09 / 88$ & 6.28 & 4.31 & 1.97 & 31 \\
$9 / 09 / 88$ & 1.50 & .017 & 1.48 & 99 \\
\hline
\end{tabular}


Additional stream discharge was lost from Hatchery and Howard Creek. Approximately $0.50 \mathrm{ft}^{3} / \mathrm{s}$ and $0.07 \mathrm{ft}^{3} / \mathrm{s}$ was lost from Hatchery and Howard creek, respectively. The total measured discharge lost in the Big Spring basin during the two day seepage study was $5.57 \mathrm{ft}^{3} / \mathrm{s}$ and the total measured discharge leaving the basin in streams was $2.93 \mathrm{ft}^{3} / \mathrm{s}$.

\section{Water Quality}

Total nitrogen concentrations in samples collected during the seepage study generally decreased downstream of the headwater sites in Roberts Creek in samples collected during the seepage study (fig. 7). At sites RC13, RC20, and RCIl total dissolved nitrogen concentrations exceeded $5.0 \mathrm{mg} / \mathrm{L}$. Between sites $\mathrm{RC10}$ and $\mathrm{RC17}$ concentrations ranged from 1.6 to $3.7 \mathrm{mg} / \mathrm{L}$. The greatest total dissolved nitrogen concentration $(7.6 \mathrm{mg} / \mathrm{L})$ was in a sample from site RC18, located downstream of the mouth of Silver Creek. Concentrations then decreased downstream to site RC2. The total dissolved nitrogen concentration of water in Roberts Creek leaving the study area was $1.0 \mathrm{mg} / \mathrm{L}$. Upstream of site RC18, the dominant nitrogen species was nitrate plus nitrite. Ammonia was the predominant nitrogen species at RC18 and organic nitrogen was the predominant species downstream of RC18.

Total nitrogen loads displayed a similar pattern to total dissolved nitrogen concentrations in Roberts Creek, greatest in the headwaters sites then decreasing downstream to site RC17 (fig.8). The load increased from $\mathrm{RCl} 7$ to RC18 and then decreased downstream in the final 9.5 miles of Roberts Creek in the study area. During the two-day seepage study, $0.13 \mathrm{t} / \mathrm{d}$ (tons per day) of total dissolved nitrogen were lost from Roberts Creek and approximately $0.01 \mathrm{t} / \mathrm{d}$ left the study area in Roberts Creek.

Total dissolved nitrogen concentrations, almost entirely as nitrate plus nitrite, were $28 \mathrm{mg} / \mathrm{L}$ at site SC10 and decreased to $8.3 \mathrm{mg} / 1$ at site SC3 (fig. 8). Downstream total nitrogen concentrations ranged from approximately 8.0 to $11.0 \mathrm{mg} / \mathrm{L}$ and the predominant nitrogen species was ammonia. Combined total dissolved nitrogen loads as nitrogen at the two upstream sites was $0.04 \mathrm{t} / \mathrm{d}$. The load decreased at the next downstream site, SC3, to $0.03 \mathrm{t} / \mathrm{d}$ and then increased downstream to $0.04 \mathrm{t} / \mathrm{d}$ at site $\mathrm{SC6}$. The load at SCl was $0.02 \mathrm{t} / \mathrm{d}$ which is a loss of approximately $0.04 \mathrm{t} / \mathrm{d}$ from the combined load of East Fork and the main stem of Silver Creek. The total measured nitrogen loss in Silver Creek was approximately $0.05 \mathrm{t} / \mathrm{d}$.

Nitrogen also was lost from Hatchery and Howard Creeks. The total measured nitrogen load decreased $0.01 \mathrm{t} / \mathrm{d}$ in Hatchery Creek and decreased approximately $0.004 \mathrm{t} / \mathrm{d}$ in Howard Creek. The total dissolved nitrogen load lost in the study area was $0.19 \mathrm{t} / \mathrm{d}$ and the dissolved nitrogen load leaving the Big Spring basin in streams was approximately $0.02 \mathrm{t} / \mathrm{d}$. 


\section{SUMMARY}

An investigation to collect hydrologic data in the Big Spring ground-water basin in northeastern Iowa was initiated in water year 1988 . The investigation was conducted in cooperation with the Iowa Department of Natural Resources, Geological Survey Bureau. The study area is located in Clayton County in northeastern Iowa in an area of karst topography and corresponds with a ground-water basin draining through Big Spring.

Information on precipitation, streams, and ground water was collected in water year 1988. Precipitation was measured at three sites and precipitation quality was determined from samples collected at Big Spring. Stream discharge was monitored at three sites and monthly water-quality samples collected at two sites on streams in the Big Spring Basin. Discharge was measured and water-quality samples collected at 38 sites on streams in the basin to determine loss of water and nutrients during a period of low streamflow. Discharge from Big Spring was monitored continuously and water-quality samples were collected monthly.

Total rainfall at Big Spring was 24.08 in. The greatest rainfall was in the month of September when 4.89 and 4.82 in. fell at sites BOOGD and RC2, respectively. Chemical analyses of precipitation indicates that calcium and sulfate are the predominant ions in solution. The median calcium concentration was $0.81 \mathrm{mg} / \mathrm{L}$ and the median sulfate concentration was $2.0 \mathrm{mg} / \mathrm{L}$. Median concentrations of nitrate and ammonia as nitrogen were 0.40 and 0.37 $\mathrm{mg} / \mathrm{L}$, respectively.

The daily mean discharge of Unnamed Creek at site BOOGD was 0.0 to 7.0 $\mathrm{ft}^{3} / \mathrm{s}$. Unnamed Creek was dry during parts of December and January and from the last week in June through the end of the water year. Calcium, magnesium, and bicarbonate are the predominant ions in the monthly samples. Nitrate plus nitrite concentrations ranged from 7.6 to $14 \mathrm{mg} / \mathrm{L}$ as nitrogen. Two pesticides, atrazine and alachlor, were detected in the four monthly samples. Atrazine was present in all samples and the concentration ranged from 0.16 to $0.34 \mu \mathrm{g} / \mathrm{L}$. The concentration of alachlor was greater than the detection level in one sample $(0.11 \mu \mathrm{g} / \mathrm{L})$.

The daily mean discharge at site RC2, Roberts Creek above Saint 0laf, ranged from 190 to $0.02 \mathrm{ft}^{3} / \mathrm{s}$. The median discharge for the 1988 water year was $7.7 \mathrm{ft}^{3} / \mathrm{s}$. Continuous monitoring indicates that $\mathrm{pH}$ and water temperature vary diurnally and are greatest during the mid-afternoon and smallest from 0600 to 0900 hours. Specific conductance varies inversely with $\mathrm{pH}$ and water temperature. Calcium, magnesium, and bicarbonate are the predominant ions in the monthly samples from site RC2. Nitrate plus nitrite concentrations ranged from 0.5 to $15 \mathrm{mg} / \mathrm{L}$ as nitrogen. Four pesticides, atrazine, cyanazine, alachlor, and metolachlor, were detected in nine samples. Total recoverable atrazine concentrations ranged from less than 0.10 to $0.72 \mu \mathrm{g} / \mathrm{L}$. Total recoverable cyanazine concentrations ranged from less than 0.10 to $0.72 \mu \mathrm{g} / \mathrm{L}$. Metolachlor $(0.11 \mu \mathrm{g} / \mathrm{L})$ and alachlor $(0.55 \mu \mathrm{g} / \mathrm{L})$ concentrations exceeded the detection limit in one sample. 
Continuous monitoring indicates that water temperature, $\mathrm{pH}$, and specific conductance were relatively constant in Big Spring for the period May 4 to September 30. The daily mean water temperature ranged from 9.7 to $10.6 \mathrm{C}$, the daily mean specific conductance ranged from 690 to $735 \mu \mathrm{S} / \mathrm{cm}$, and the daily median $\mathrm{pH}$ ranged from 6.65 to 7.10 units. The predominant ions in solution are calcium, magnesium, and bicarbonate. Dissolved nitrate plus nitrite concentrations ranged from 7.5 to $11 \mathrm{mg} / \mathrm{L}$. Atrazine was the only pesticide present in concentrations greater than the detection limit of $0.10 \mu \mathrm{g} / \mathrm{L}$. Concentrations ranged from less than $0.10 \mu \mathrm{g} / \mathrm{L}$ to $0.26 \mu \mathrm{g} / \mathrm{L}$.

A seepage study was conducted during a period of low streamflow from June 28 to June 29 to determine gaining and losing reaches of streams and to determine changes in nutrient concentrations and loads in streams. The total measured discharge lost in the Big Spring basin during the seepage study was $5.57 \mathrm{ft}^{3} / \mathrm{s}$ and the total measured discharge leaving the basin through streamflow was $2.93 \mathrm{ft}^{3} / \mathrm{s}$. The total dissolved nitrogen load lost was 0.19 $t / d$ and the total dissolved nitrogen load leaving the basin in streams was $0.02 t / d$. 


\section{SELECTED REFERENCES}

Buchanan, T.J., and Somers, W.P., 1969, Discharge measurements at gaging stations: U.S. Geological Survey Techniques of Water-Resources Investigations, Book 3, Chapter A8, 65 p.

Guy, H.P., and Norman, V.W., 1970, Field methods for measurement of fluvial sediment: U.S. Geological Survey Techniques of Water-Resources Investigations, Book 3, Chapter C2, 59 p.

Hallberg, G.R., Hoyer, B.E., Bettis, E.A., and Libra, R.D., 1983, Hydrogeology, water quality, and land management in the Big Spring basin, Clayton County, Iowa: Iowa Geological Survey Bureau Open File Report 83-3, 191 p.

Hallberg, G.R., Libra, R.B., Bettis, E.A., and Hoyer, B.E., 1984 , Hydrogeologic and water quality investigations in the Big Spring Basin, Clayton County, Iowa: Iowa Geological Survey Bureau Open-File Report $84-4,231$ p.

Kennedy, D.J., 1983, Computation of continuous records of streamflow: U.S. Geological Survey Techniques of Water-Resources Investigations, Book 3, Chapter A13, $53 \mathrm{p}$.

Kilpatrick, F.A., and Schneider, V.R., 1983, Use of flumes in measuring discharge: U.S. Geological Survey Techniques of Water-Resources Investigations; Book 3, Chapter A14, 46 p.

Libra, R.D., Hallberg, G.R., Hoyer, B.E., and Johnson, L.G., 1986 , Agricultural impacts on ground water quality: The Big Spring Basin study, Iowa: Proceedings of the Agricultural Impacts on Ground Water Conference, p. 253-373.

National Atmospheric Deposition Program/ National Trends Network, 1988, Instruction Manual NADP/NTN site operation: Natural Resource Ecology Laboratory Colorado State University.

National Oceanic and Atmospheric Administration, 1988, Climatological data annual summary Iowa 1987; U.S. Department of Commerce, vol. 98, no. $13,30 \mathrm{p}$. 
Rantz, S.E., and others, 1982a, Measurement and computation of streamflow: Volume 1. Measurement of stage and discharge: U.S. Geological Survey Water-Supply Paper 2175, p 1-284. , 1982b, Measurement and computation of streamflow: Volume 2. computation of discharge: U.S. Geological Survey Water-Supply Paper 2175 , p 285-631.

U.S. Environmental Protection Agency, 1983, Methods for chemical analysis of water and wastes: Cincinnati, Ohio, EPA-600/4-79-020.

, 1986, Maximum contaminant levels (subpart B of part 141, National interim primary drinking-water regulations): U.S. Code of Federal Regulations, Title 40, Parts 100 to 149 , revised as of July 1, 1986, p.524-528.

Wood, W.W., 1976, Guidelines for collection and field analysis of groundwater samples for selected unstable constituents: U.S. Geological Survey Techniques of Water-Resources Investigations, Book 1, Chapter D1, 24 p. 